\title{
Evaluating and Prioritizing Antimicrobial Stewardship Programs for Nursing Homes: A Modified Delphi Panel
}

by

Shaul Zvi Kruger

A thesis submitted in conformity with the requirements for the degree of Master of Science Institute of Medical Science University of Toronto

(C) Copyright by Shaul Zvi Kruger 2018 


\title{
Evaluating and Prioritizing Antimicrobial Stewardship Programs for Nursing Homes: A Modified Delphi Panel
}

\author{
Shaul Zvi Kruger \\ Master of Science \\ Institute of Medical Science \\ University of Toronto
}

2018

\section{Abstract}

Antibiotic use in nursing homes (NHs) is often inappropriate and linked to adverse events and antimicrobial resistance (AMR). Antimicrobial stewardship programs (ASPs) are initiatives that optimize antibiotic use by minimizing inappropriate use, treatment cost, and the overall spread of AMR. NH providers and residents are candidates for ASP implementation; yet, guidelines for implementation are limited. We utilized the Modified Delphi Method to provide NH providers with 2 tools: (1) a prioritized list of necessary ASPs, and (2) resources for implementation. 16 panelists completed two survey rounds, attended an in-person meeting, and evaluated 15 ASP interventions across several criteria. 6 interventions were prioritized for NHs: (1) guidelines for empiric prescribing, (2) audit and feedback, and communication tools (tied), (3) short course antibiotic therapy, (4) scheduled antibiotic reassessment, and (5) clinical decision support systems. Interventions may need to be delivered using a multifaceted approach; however, this is likely infeasible for nursing homes. 


\section{Acknowledgements}

Firstly, I would like to thank my Master's supervisor, Dr. Chaim Bell, for his endless support and all his hard work in bringing this thesis to completion. Dr. Bell's incredible ability to manage being the Physician-in-Chief at Mount Sinai Hospital and supervising multiple Master's and PhD graduate students is inspiring. My achievements would not have been possible without his help and I am eternally grateful.

I would also like to acknowledge my unofficial co-supervisor, Dr. Andrew Morris. He played a critical role in our Modified Delphi panel as the facilitator, and was also a ceaseless source of guidance, not to mention humorous conversation!

Thank you to my Program Advisory Committee: Drs. Susan Bronskill and Lianne Jeffs. Dr. Bronskill, for her consistent and invaluable evaluation of my thesis progress. Dr. Jeffs, for sharing her creativity and ingenious ideas, and for including me in her qualitative research. Thank you both for your guidance and much-needed pressure to get this done. Our PAC meetings were so much more enjoyable than I could have anticipated!

I would also like to thank the UHN/Sinai ASP Team for all the interesting meetings and wonderful discussion of novel ASP projects. Particularly, I want to thank Mrs. Marilyn Steinberg, for all of her administrative support and assistance when I needed it most.

I would like to acknowledge the individuals who participated in our Modified Delphi panel; without them, this thesis would have been impossible: Dr. Carla Beaton, Ms. Anne Bialachowski, Ms. Sharron Cooke, Dr. Nick Daneman, Dr. Kamyab Ghatan, Ms. Devora Greenspon, Dr. Lynn Johnston, Dr. Louis Kennedy, Ms. Maloree Kubica, Mr. Jonathan Lam, Dr. Bradley Langford, Ms. Dee Lender, Dr. Justin Lin, Dr. Mark Loeb, Dr. Lindsay Nicolle, and Dr. Lisa Sever. Thank you all for taking your time to complete both surveys and attend the full-day Delphi meeting.

Lastly, I want to thank my family for their support and encouragement. Mom and Dad, your boundless love and belief in me is my motivation. 


\section{Table of Contents}

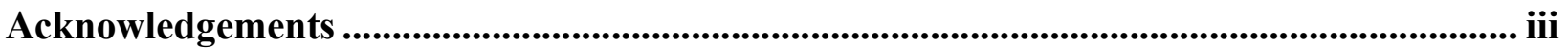

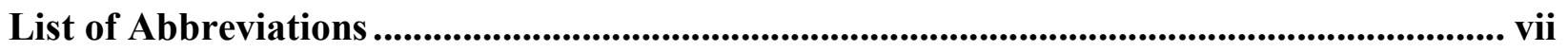

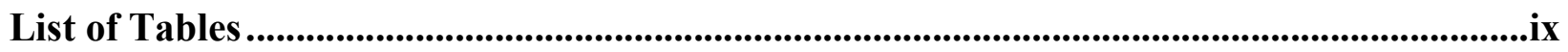

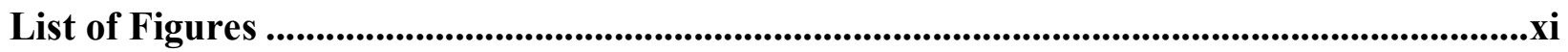

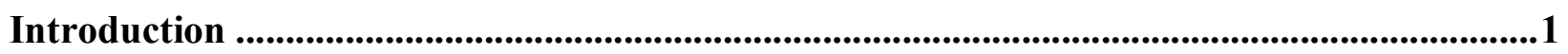

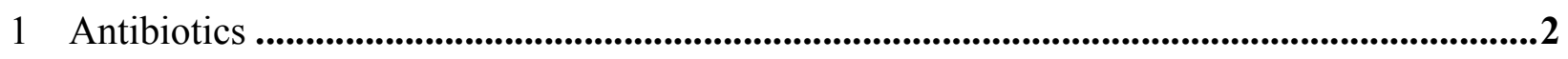

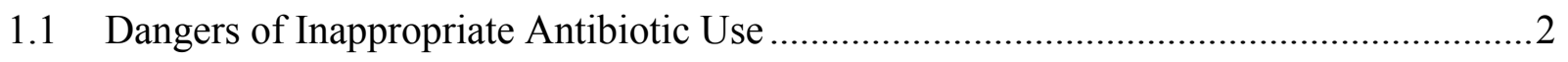

2 Antibiotics in Nursing Homes and Adverse Drug Events ..................................................4

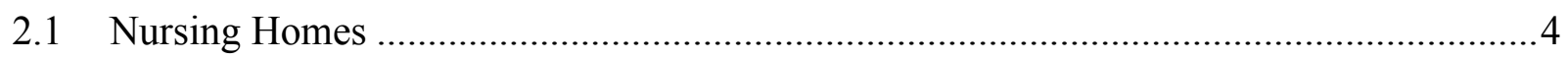

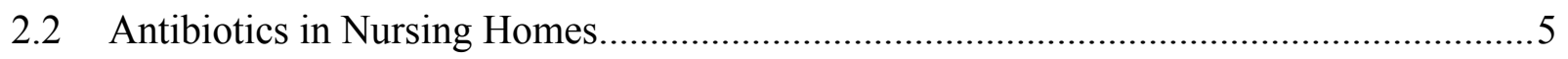

2.3 Adverse Drug Events and Reactions in Nursing Homes ........................................6

3 Inappropriate Antibiotics in Nursing Homes...................................................................7

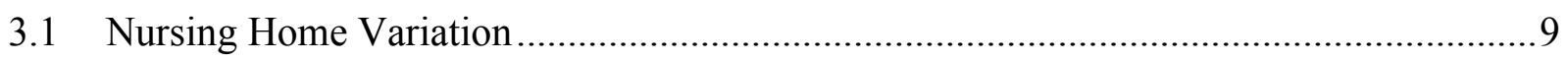

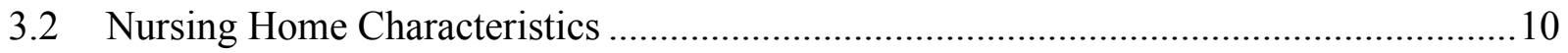

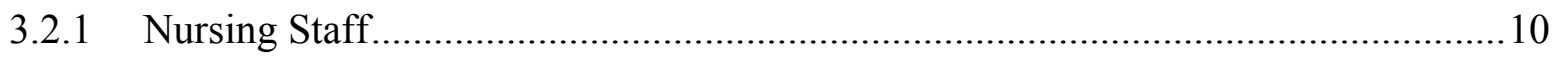

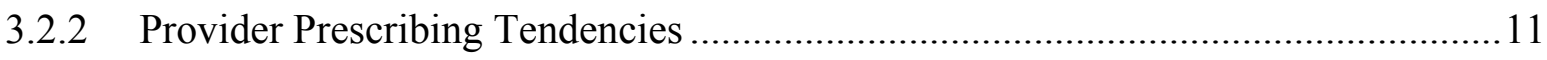

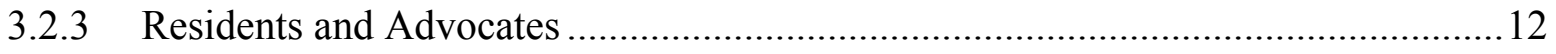

3.2.4 Infection, Prevention, and Control Resources ............................................... 12

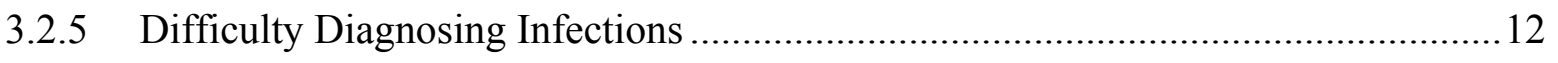

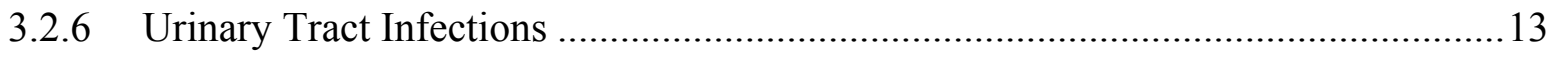

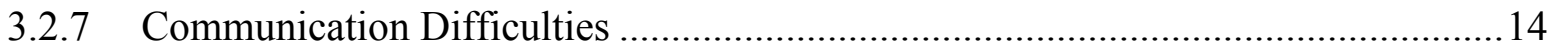

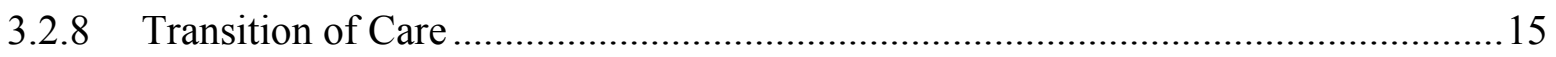

4 Improving Antibiotic Use .................................................................................................................16

4.1 Antimicrobial Stewardship Programs (ASPs).................................................... 16

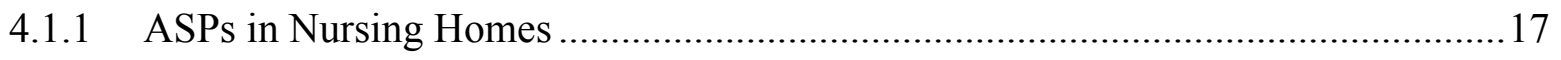

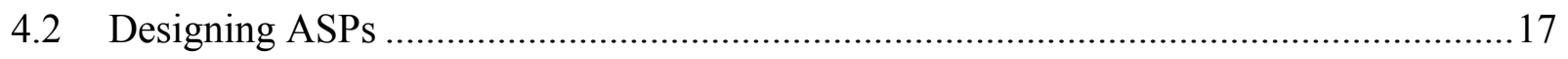

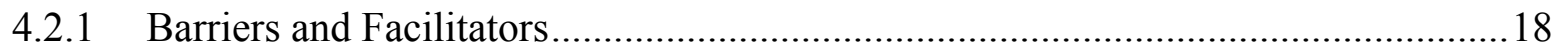




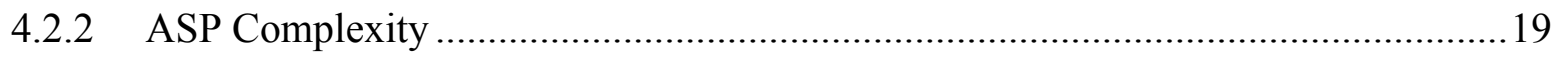

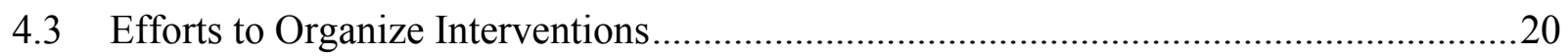

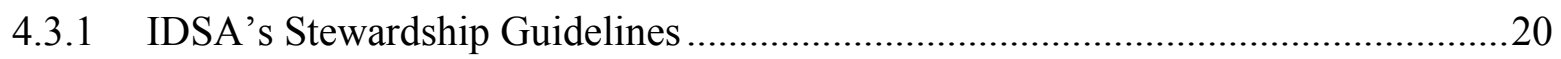

4.3.2 Public Health Ontario's ASP Strategies …………….......................................22

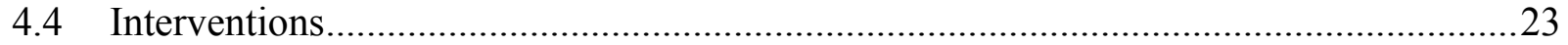

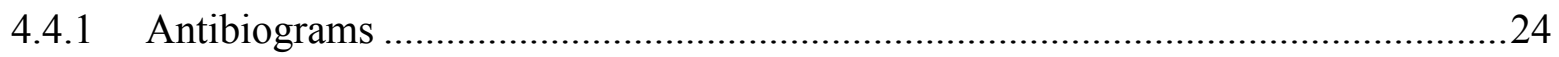

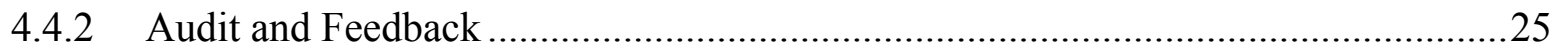

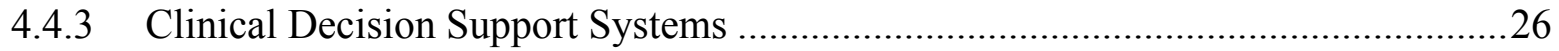

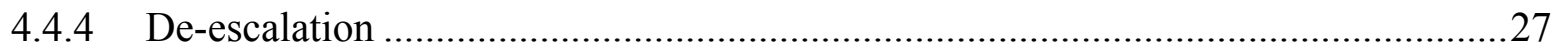

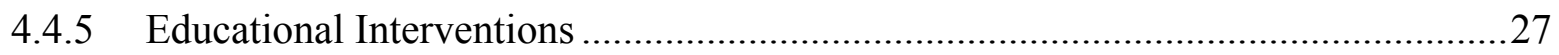

4.4.6 Formulary Interventions and Antibiotic Cycling........................................................30

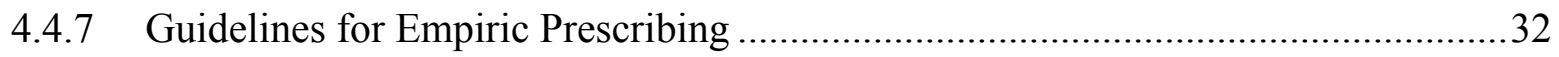

4.4.8 Intravenous to Oral Antibiotic Conversion............................................................. 32

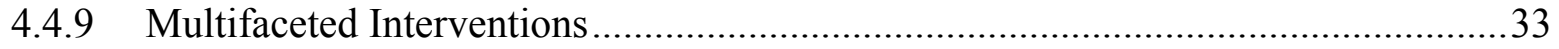

4.4.10 Short Course Antibiotic Therapy............................................................................. 35

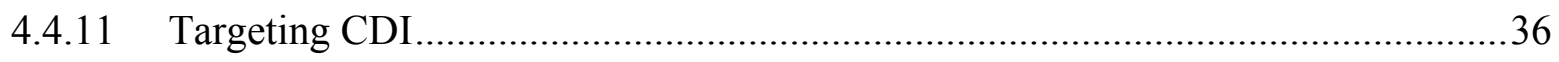

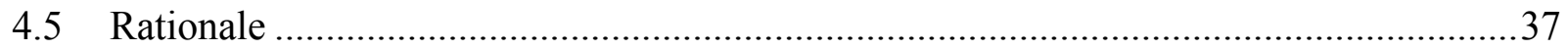

5 Conclusions ..............................................................................................................................................38

6 Study Purpose, Objectives, and Intended Outcomes..........................................................................40

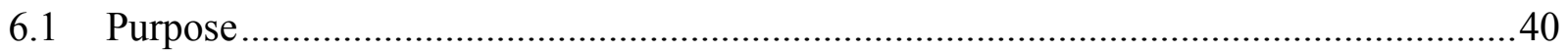

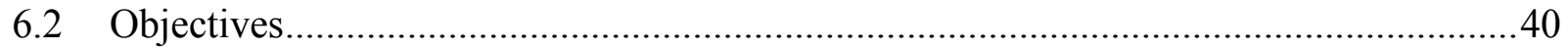

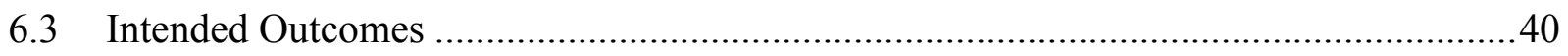

Materials and Methods ............................................................................................................................41

7 The Modified Delphi Method......................................................................................................................42

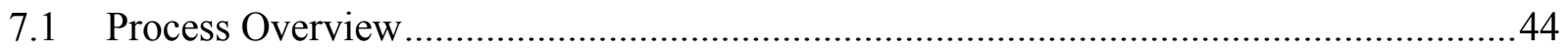

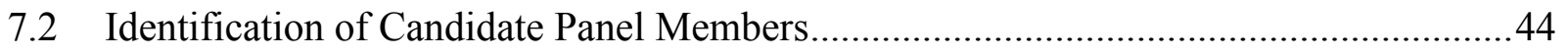

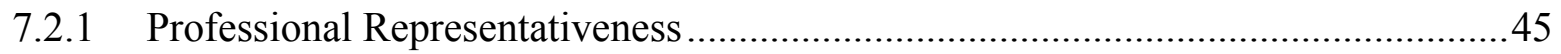

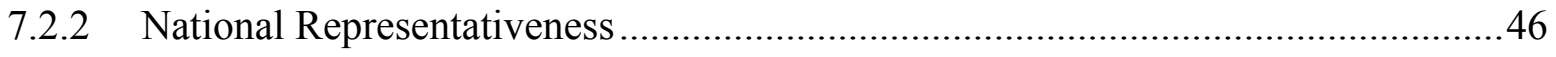

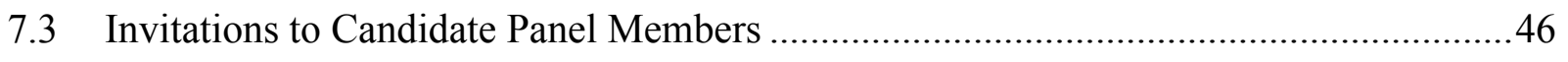

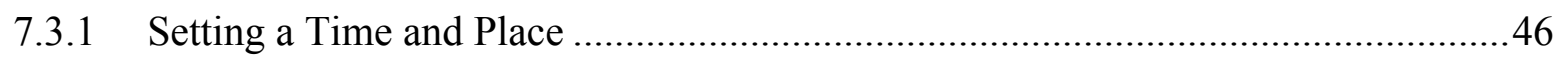

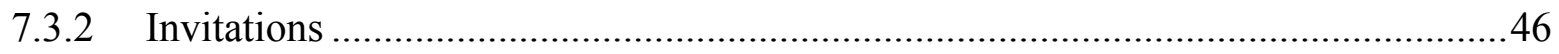




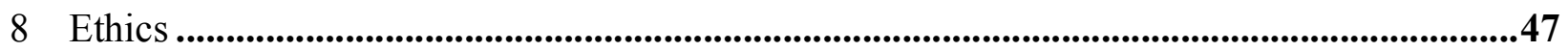

9 Background Package .......................................................................................................................................48

9.1 Introduction and Brief Literature Summary...............................................................

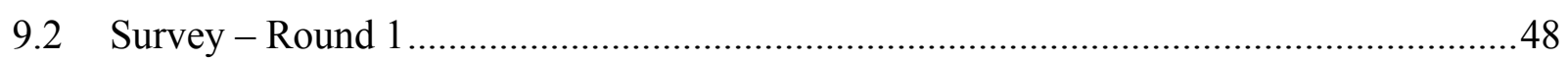

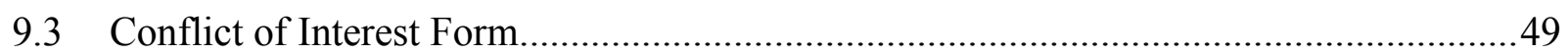

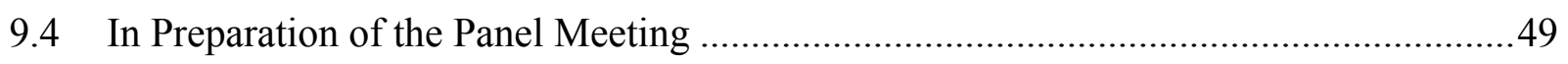

10 Panel Day......................................................................................................................................49

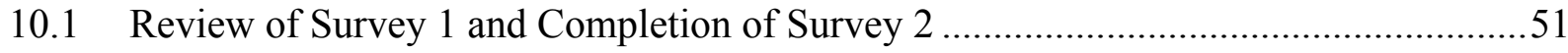

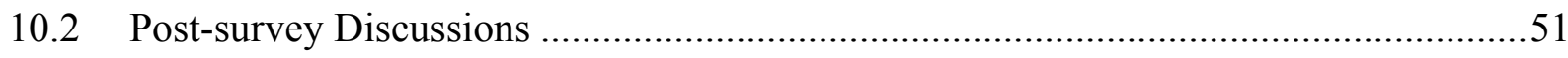

10.2.1 Final List of ASP Interventions for Nursing Homes ............................................51

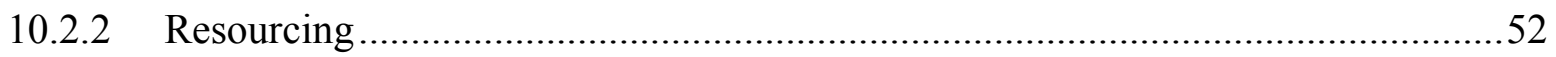

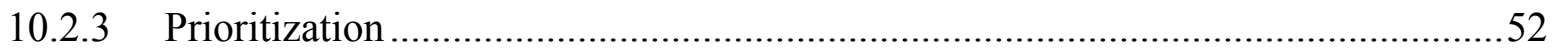

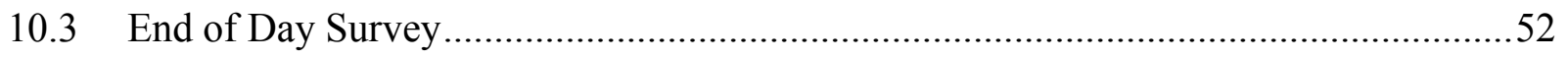

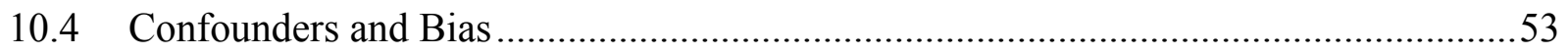

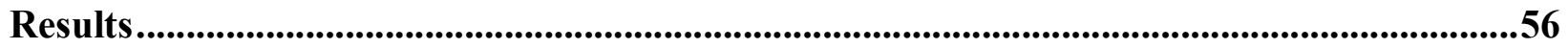

11 Modified Delphi Panel Members .........................................................................................................57

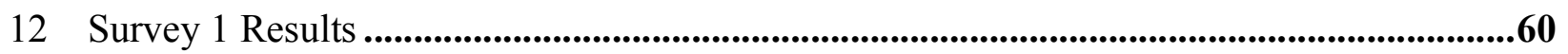

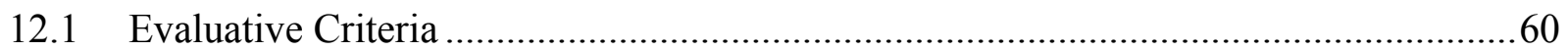

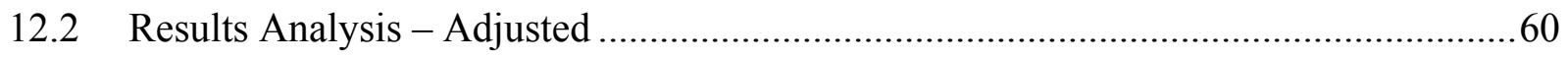

12.2.1 Guidelines for Empiric Prescribing .....................................................................61

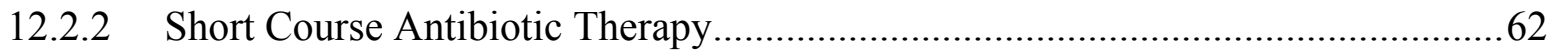

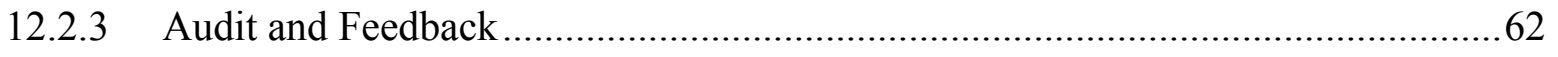

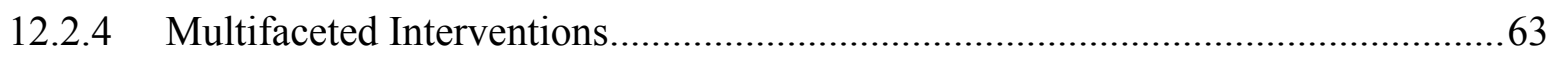

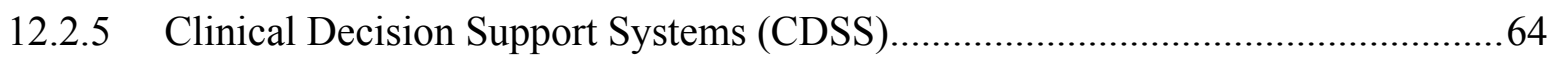

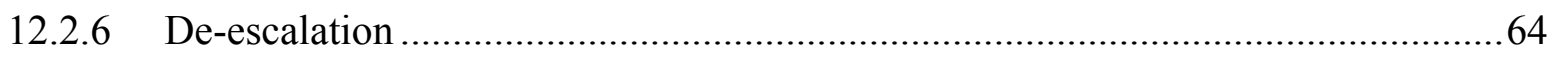

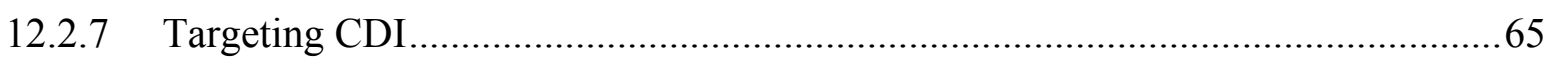

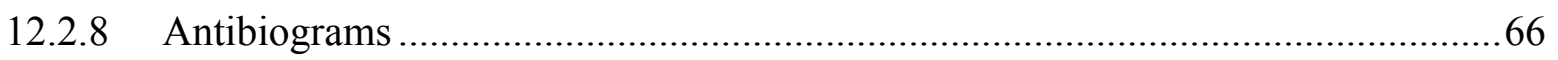

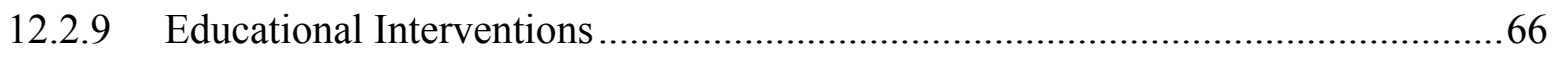

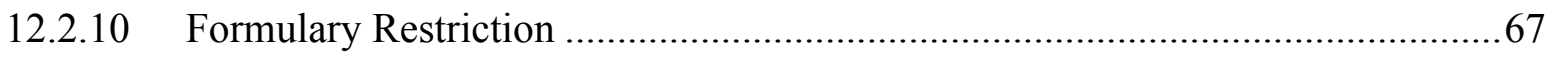

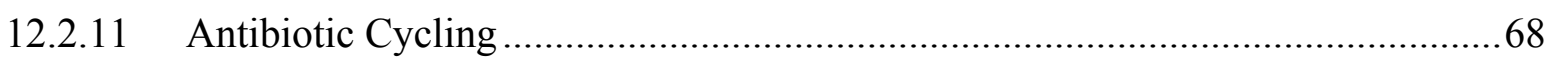

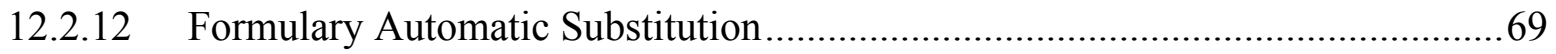




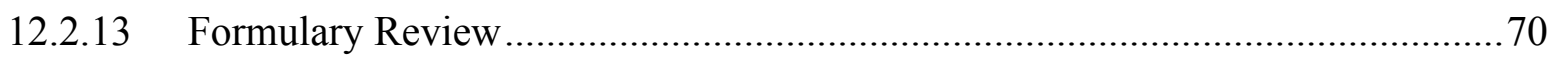

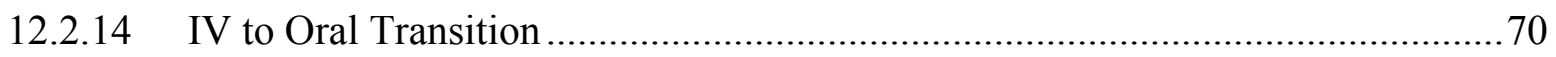

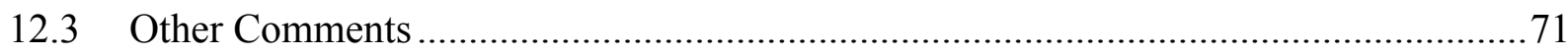

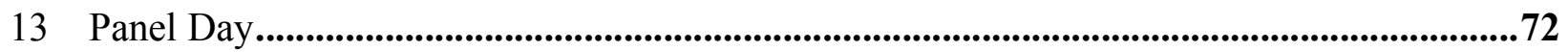

13.1 Survey 1 Review and Intervention Elimination Phase 1 ........................................... 72

13.2 Survey 2 and Intervention Elimination Phase 2...................................................... 73

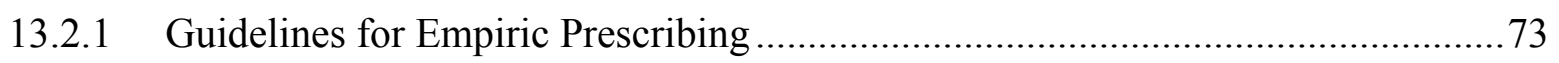

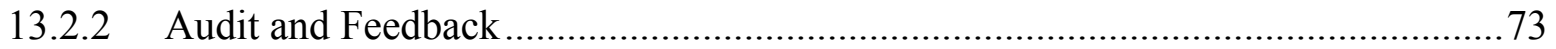

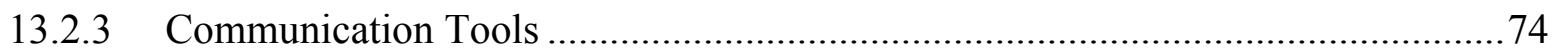

13.2.4 De-Escalation (Renamed Scheduled Antibiotic Reassessment)..............................74

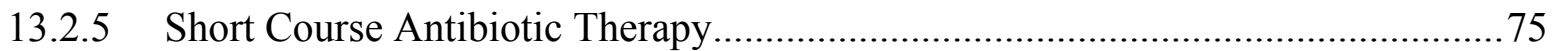

13.2.6 Clinical Decision Support Systems ..................................................................... 76

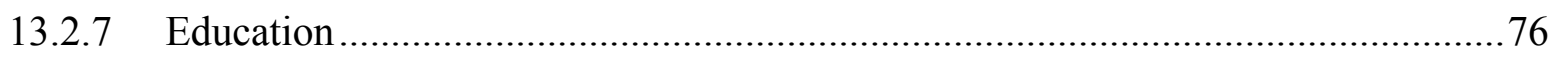

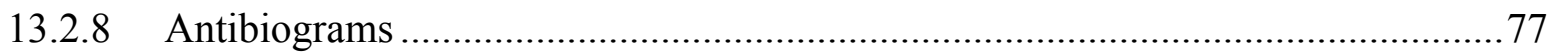

13.2.9 Formulary Automatic Substitution ....................................................................

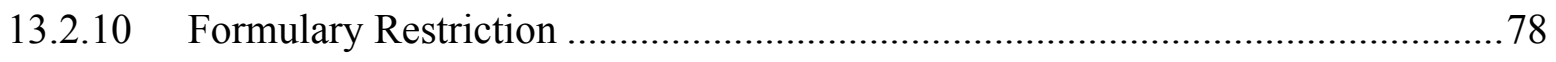

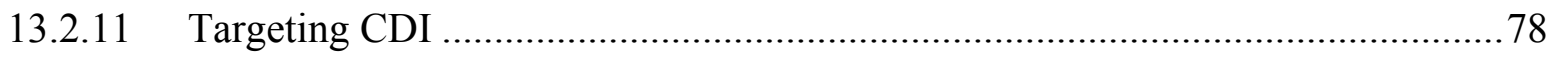

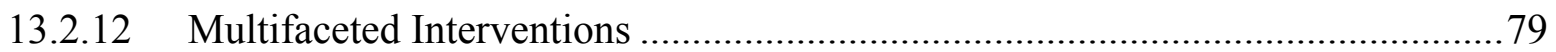

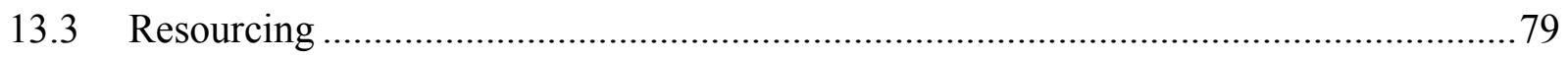

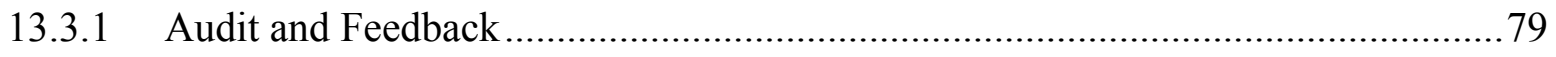

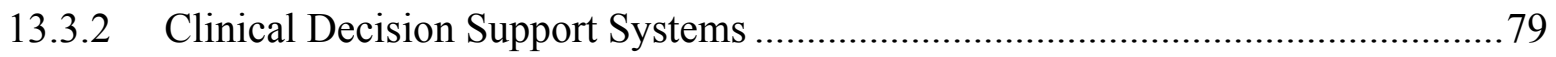

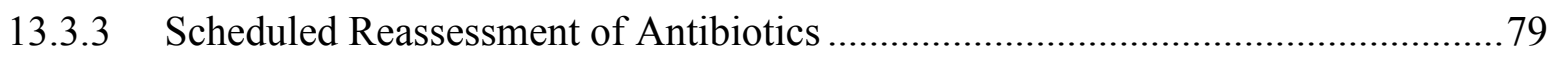

13.3.4 Guidelines for Empiric Prescribing ................................................................... 79

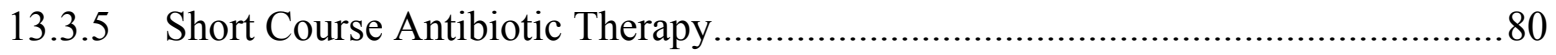

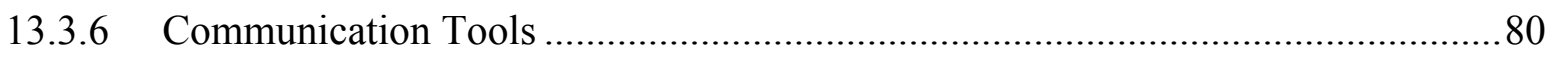

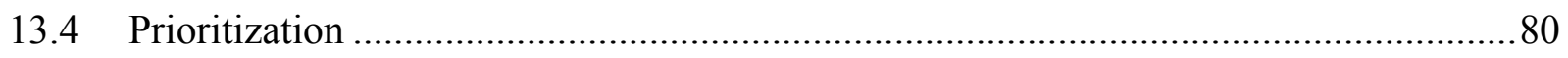

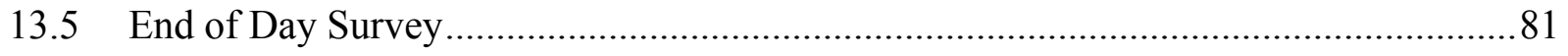

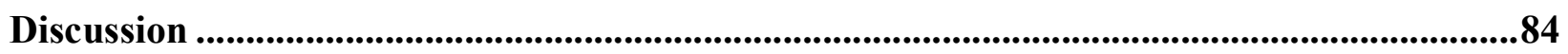

14 Overview ............................................................................................................................................85

15 Summary of Results...........................................................................................................................85

16 Elimination of ASP Interventions....................................................................................................86 


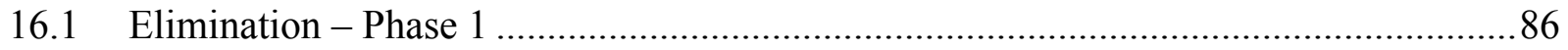

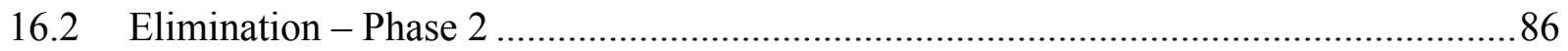

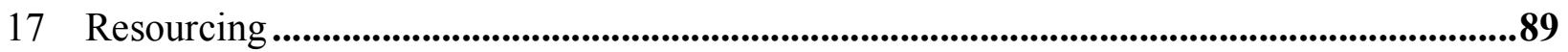

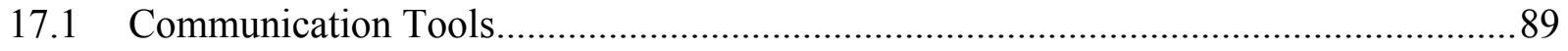

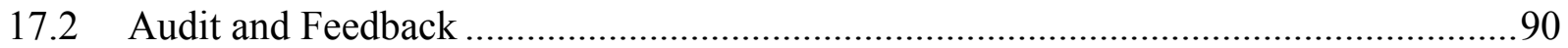

17.3 Clinical Decision Support Systems..........................................................................

$17.4 \quad$ Scheduled Antibiotic Reassessment ……………….................................................92

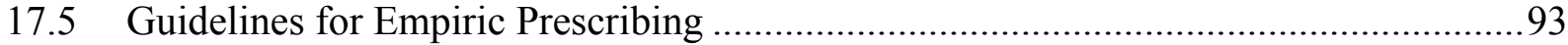

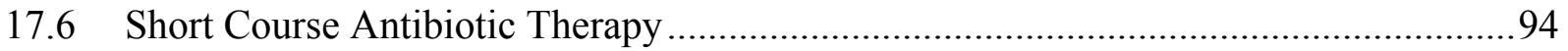

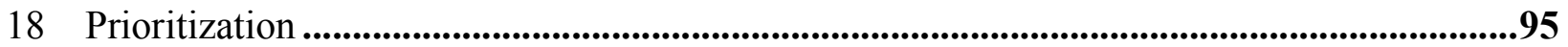

19 Interpretation of Results ..........................................................................................................98

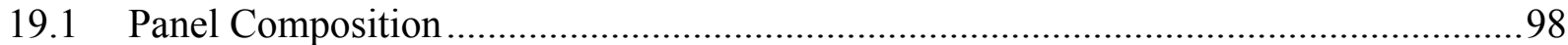

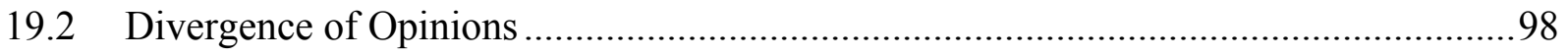

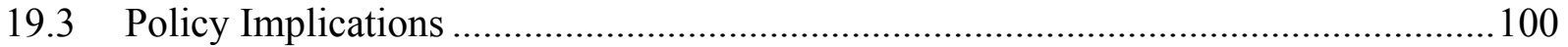

20 Strengths and Limitations .............................................................................................................101

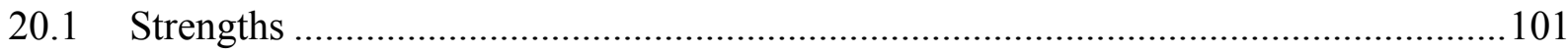

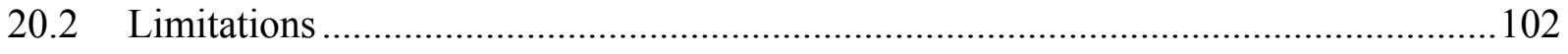

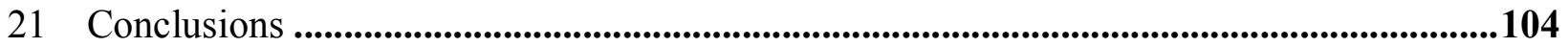

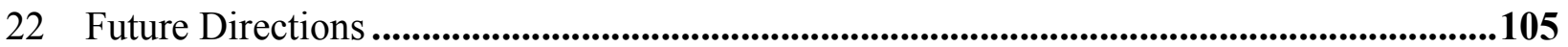

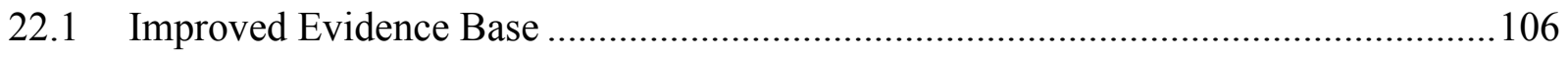

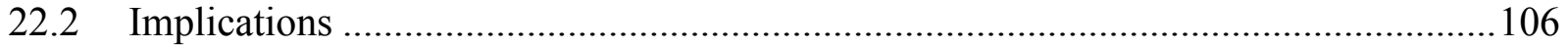

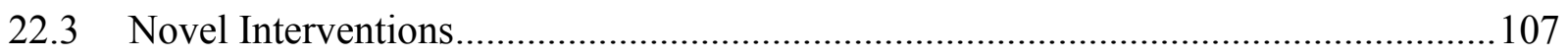

Bibliography ...............................................................................................................109

Appendix A. Background Package ....................................................................................................130

Appendix B. Survey 1 (paper version) ...........................................................................................134

Appendix C. Conflict of Interest Form.............................................................................................150

Appendix D. Delphi Meeting Agenda ...........................................................................151

Appendix E. End of Day Survey................................................................................................152 
Appendix F. Literature Search Strategy ...............................................................................154

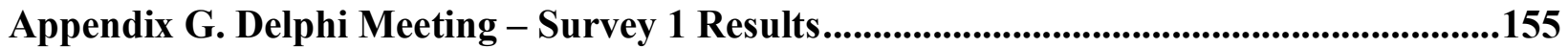

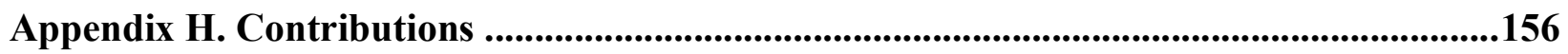

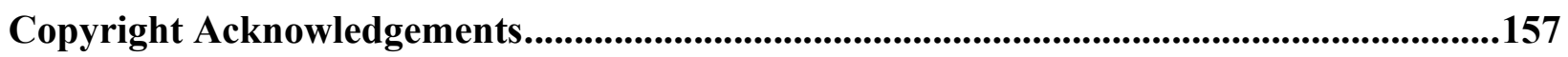




\section{List of Abbreviations}

\begin{tabular}{|c|c|}
\hline ADE & Adverse Drug Event \\
\hline ADR & Adverse Drug Reaction \\
\hline AMR & Antimicrobial Resistance \\
\hline ASP & Antimicrobial Stewardship Program \\
\hline CAHLTC & Canadian Association for Long Term Care \\
\hline $\mathrm{CDC}$ & Centers for Disease Control and Prevention \\
\hline CDI & Clostridium difficile Infection \\
\hline CDSS & Computerized Decision Support Systems \\
\hline CIHI & Canadian Institute for Health Information \\
\hline CPG & Clinical Practice Guidelines \\
\hline ED & Emergency Department \\
\hline EMR & Electronic Medical Record \\
\hline $\mathrm{FCO}$ & Family Councils Ontario \\
\hline GDP & Gross Domestic Product \\
\hline GI & Gastrointestinal \\
\hline GRADE & Grading of Recommendations Assessment, Development and Evaluation \\
\hline GTA & Greater Toronto Area \\
\hline $\mathrm{HCP}$ & Health Care Provider \\
\hline IC & Infection Control \\
\hline ICES & Institute for Clinical Evaluative Sciences \\
\hline ID & Infectious Disease \\
\hline IDSA & Infectious Disease Society of America \\
\hline IPAC & Infection Prevention and Control \\
\hline IUC & Indwelling Urinary Catheter \\
\hline IV & Intravenous \\
\hline LPN & Licensed Practical Nurse \\
\hline LTCHA & Long Term Care Homes Act \\
\hline MDRO & Multidrug-resistant Organism \\
\hline MOHLTC & Ministry of Health and Long-Term Care \\
\hline NBI & Needed but Unnecessary \\
\hline NICE & National Institution of Clinical Excellence \\
\hline
\end{tabular}


OARC Ontario Association of Residents' Council

ODB Ontario Drug Benefit

OLTCA Ontario Long Term Care Association

OLTCC Ontario Long Term Care Clinicians

OLTCP Ontario Long Term Care Physicians

OR Odds Ratio

PHO Public Health Ontario

RAM RAND/UCLA Appropriateness Method

RAND Research and Development

RCT Randomized Controlled Trial

RN Registered Nurse

RPN Registered Practical Nurse

RTI Respiratory Tract Infection

SBAR Situation-Background-Assessment-Recommendation

SHEA Society for Healthcare Epidemiology of America

UTI Urinary Tract Infection

WHO World Health Organization 


\section{List of Tables}

Table 1 Summary of ASP Interventions and their Descriptions

Table 2 Modified Delphi Panel Participant Representation

Table 3 Survey 1 Results for Guidelines for Empiric Prescribing Across Five Evaluative Criteria

Table 4 Survey 1 Results for Short Course Antibiotic Therapy Across Five Evaluative Criteria

Table 5 Survey 1 Results for Audit and Feedback Across Five Evaluative Criteria

Table 6 Survey 1 Results for Multifaceted Interventions Across Five Evaluative Criteria

Table 7 Survey 1 Results for Clinical Decision Support Systems Across Five Evaluative Criteria

Table $8 \quad$ Survey 1 Results for De-escalation Across Five Evaluative Criteria

Table 9 Survey 1 Results for Targeting CDI Across Five Evaluative Criteria

Table 10 Survey 1 Results for Antibiograms Across Five Evaluative Criteria

Table 11 Survey 1 Results for Education Across Five Evaluative Criteria

Table 12 Survey 1 Results for Formulary Restriction Across Five Evaluative Criteria

Table 13 Survey 1 Results for Antibiotic Cycling Across Five Evaluative Criteria

Table 14 Survey 1 Results for Formulary Automatic Substitution Across Five Evaluative Criteria

Table 15 Survey 1 Results for Formulary Review Across Five Evaluative Criteria

Table 16 Survey 1 Results for IV to Oral Transition Across Five Evaluative Criteria

Table 17 Survey 2 Results for Guidelines for Empiric Prescribing Across Five Evaluative Criteria

Table 18 Survey 2 Results for Audit and Feedback Across Five Evaluative Criteria

Table 19 Survey 2 Results for Communication Tools Across Five Evaluative Criteria 
Table 20 Survey 2 Results for Scheduled Antibiotic Reassessment Across Five Evaluative Criteria

Table 21 Survey 2 Results for Short Course Antibiotic Therapy Across Five Evaluative Criteria

Table 22 Survey 2 Results for Clinical Decision Support Systems Across Five Evaluative Criteria

Table 23 Survey 2 Results for Education Across Five Evaluative Criteria

Table 24 Survey 2 Results for Antibiograms Across Five Evaluative Criteria

Table 25 Survey 2 Results for Formulary Automatic Substitution Across Five Evaluative Criteria

Table 26 Survey 2 Results for Formulary Restriction Across Five Evaluative Criteria

Table 27 Survey 2 Results for Targeting CDI Across Five Evaluative Criteria

Table 28 Prioritization of ASP Interventions for Nursing Homes

Table 29 End of Day Survey Results 


\section{List of Figures}

Figure 1 Some Causes of Inappropriate Antibiotic Prescriptions in Nursing Homes

Figure 2 The GRADE System for rating quality of evidence and determining the strength of recommendations.

Figure 3 The RAND/UCLA Appropriateness Method (RAM)

Figure 4 Overview of the Modified Delphi Process

Figure 5 Modified Delphi panel recruitment process 


\section{List of Appendices}

Appendix A Background Package

Appendix B Survey 1 (paper version)

Appendix C Conflict of Interest Form

Appendix D Delphi Meeting Agenda

Appendix E End of Day Survey

Appendix F Literature Search Strategy

Appendix G Delphi Meeting - Survey 1 Results

Appendix $\mathrm{H}$ Contributors 


\section{Chapter 1 Introduction}

\section{Key Objectives}

(1) To provide an overview of the literature describing inappropriate antibiotic prescriptions in health care,

(2) To describe the nursing home setting, inappropriate use of antibiotics in nursing homes, and the causes of inappropriate prescriptions, and

(3) To present a review of efforts to improve antibiotic prescriptions, Antimicrobial Stewardship Programs, and their implementation (or absence) in nursing homes. 


\section{$1 \quad$ Antibiotics}

Antibiotics are considered one of the most important and revolutionary medical discoveries of all time. Since their discovery in the early $20^{\text {th }}$ century by Sir Alexander Fleming, antibiotics have facilitated the cure of many infections (Gualerzi et al., 2013). However, antibiotics are losing their efficacy and these invaluable resources may be lost to antimicrobial resistance (AMR). Antibiotic development has essentially stopped; few antibiotics are in the process of development to manage the spread of AMR. A reason for the lack of research may be the unprofitability of developing new antibiotics, although other factors have also contributed to the lack of novel antibiotic development, such as the development of AMR prior to public release and cost issues in comparison to other medications (Burki, 2017). Current antibiotics are effective for treating infection and demand for antibiotics drops entirely once the infection is eradicated; this contrasts with the profitability of medications for treating chronic illnesses, which are often used for the remainder of a patient's life (Braine, 2011; Infectious Diseases Society of America (IDSA), 2004). With increasing numbers of resistant bacteria and reduced production of novel antibiotics, prescribers are left with fewer and sometimes no options for effectively treating patients. Thus, action must be taken to ensure that antibiotics do not lose their efficacy and AMR is prevented (Hughes, 2011; Wenzel, 2004).

\subsection{Dangers of Inappropriate Antibiotic Use}

Antibiotics promote the spread of antibiotic resistant bacteria via selective pressure; antibiotics eradicate susceptible bacteria but leave the resistant bacteria to propagate (Kohanski et al., 2010). Antibiotic-resistant bacteria are becoming increasingly widespread, resulting in greater utilization of last-resort antibiotics, such as polymyxin B and colistin, for treating infections (Morrill et al., 2016; Roberts et al., 2015). Antibiotic resistance is directly linked to increased fatality (Drinka et al., 2011), length of hospital stay, and cost of treatment (Spellberg, 2008). Certain factors contribute to a patient's increased susceptibility to acquiring resistant bacteria.

Such factors include (but are not limited to) multiple medication prescriptions and environmental contamination. However, the driving force of bacterial resistance is the use of antibiotics, particularly, inappropriate antibiotic use (Centers for Disease Control and Prevention (CDC) US, 2013; Bonomo, 2000; Hughes, 2011; Nicolle, 2000). 
The mechanism for antibiotics driving the spread of AMR is through its impact on the microbiome. The human gastrointestinal (GI) tract is inhabited by up to 100 trillion microorganisms, including bacteria, viruses, and fungi. These microorganisms collectively constitute the microbiome. The bacteria in the microbiome are essential for normal GI development and provide essential amino acids and vitamins (Turnbaugh et al., 2007; Barbara G, 2016). Antibiotics are capable of altering the gut microbiome and inciting the replacement of beneficial and necessary bacterial colonizers with more dangerous bacteria (Cai et al., 2012; Iizumi et al., 2017).

Unnecessary antibiotics are viewed as 'time bombs' (Drinka et al., 2004): they can promote the spread of Clostridium difficile infection (CDI) caused by a strain of bacterium that is highly resistant to antibiotic treatment and other antibiotic resistant organisms. Although associated with inappropriate use, CDI can also spread with appropriate antibiotic use (Nicolle, 2000). Widespread antibiotic resistance will be catastrophic for both patients and the economy (directly and indirectly) if it is not prevented (O’Neill, 2014). The impact of AMR on the annual global gross domestic product (GDP) was estimated using World Bank simulation tools. The simulations included two scenarios: (1) low AMR impacts, and (2) high AMR impacts. In the low impact scenario, it was estimated that by 2050 , the annual global GDP would decrease by 1.1 percent with an annual shortfall of $\$ 1$ trillion yearly after 2030 . In the high impact scenario, it was estimated that by 2050 , the annual global GDP would decrease by 3.8 percent with an annual shortfall of $\$ 3.4$ trillion yearly after 2030 . Such a decrease would be especially noticeable in low-income countries. In either scenario, AMR is predicted to negatively impact the economy. Thus, preventing the spread of AMR is essential (Adeyi et al., 2017).

The United States economy is adversely affected by more than $\$ 20$ billion of cost yearly as a result of AMR. Although the costs are extensive, communicating the intangible expense and collateral damage due to unnecessary antibiotics to health care professionals can be challenging (Roberts et al., 2009). Rates of AMR in Canada are similar to that of other developed countries (CDC, 2013; ECDC, 2016).

Beyond increased susceptibility to resistant bacteria, inappropriate antibiotic use is also associated with increased risk of adverse drug events (ADEs) and overall health care expense (Nicolle, 2000). The dangers associated with inappropriate antibiotic use are not limited to the 
treated patient. Inappropriate antibiotic use endangers individual patients, both treated and untreated, and overall populations through increased selection of antibiotic resistant bacteria, which may have a significant impact on morbidity and mortality (Dellit et al., 2007; Hughes, 2011). Environmental contamination and cross-transmission of infection between patients threatens patients at a system level. As patients reside in a facility and transfer between institutions, they are exposed to a plethora of bacterial organisms; these bacteria are then transferred with the patients as they move to the next facility, exposing the other residents to the new bacterial organisms (Nicolle, 2000). Whether treated patients are affected more than the surrounding population or vice versa is a contested issue. Regardless, inappropriate antibiotics impact everyone by promoting the spread of AMR and the increased risk of morbidity and mortality; therefore, efforts are needed to ensure antibiotics are used only when necessary (Harbarth, 2001; Donnan et al., 2004).

There is good news about antibiotic resistance, however: it may be reversible. Refraining from antibiotic use can lead to exponential decay of resistant bacteria (Costelloe et al., 2010). Poorly designed decision-systems, such as drug-charts and out-of-date guidelines (McCaig, 1995), contribute to inappropriate antibiotics. Thus, improving antibiotic use may require examining and modifying present decision-making factors in particular health care settings (Charani et al., 2010).

\section{Antibiotics in Nursing Homes and Adverse Drug Events}

\subsection{Nursing Homes}

Nursing homes, also known as long term care facilities, are institutions that provide 24-hour care to residents (typically seniors) who do not need hospital care (Canadian Institute for Health Information (CIHI), 2014). Nursing homes are the fastest growing sect of health care sector in high-income countries. Two to five percent of the Western World's older adult population lives in nursing homes. Older adult citizens are expected to account for $20.1 \%$ of the western population by 2024 and 25\% by 2036 (Ribbe et al., 1997). There are approximately 15,000 nursing homes in the US, caring for about 1.5 million residents (National Nursing Home Survey, 2004). A 2012 survey of health spending in Canada estimated that there were 1,360 nursing 
homes in Canada (in 2012) caring for approximately 143,000 residents. There are approximately 255,000 nursing home beds in Canada; this number is expected to double by 2035. Ontario alone is home to over 600 nursing homes and over 77,000 nursing home beds. Total expenses as a result of nursing home care in Canada are an estimated \$9.8 billion (CIHI, 2014; Mody et al., 2005; Long-Term Care Utilization Report, 2018).

Nursing homes residents are highly susceptible to infections. More than 2 million infections are diagnosed in the US yearly. Infections are the greatest cause of hospitalizations in nursing homes, accounting for $27-63 \%$ of hospitalizations. Approximately 3-15\% of residents acquire an infection during their stay in a nursing home facility (Smith et al., 2008; Mody, 2007). An estimated 1 in 3 nursing home residents will acquire or be contaminated with a multidrugresistant organism (MDRO) during their stay in a facility (Trick et al., 2001).

The reasons for the high susceptibility of nursing home residents to resistant bacteria are manifold. Nursing home residents represent the oldest and most frail groups in local communities. Frailty and dependency are factors that may increase a resident's risk of being prescribed inappropriate antibiotics (High et al., 2009; Nicolle, 2014). Approximately 95\% of Canadian nursing home residents require some form of assistance with activities of daily living, such as feeding, bathing, and eating; 80\% require extensive assistance (CIHI, 2014). Such frailty and dependence is related to difficulties determining infections in nursing homes. Determining an infection in a nursing home resident is a challenging process even for well-experienced clinicians, as comorbid conditions and frailty may skew identification of infection. Additionally, selecting the appropriate antibiotic is a complicated task. Patient-related factors, such as age and polypharmacy, need to be considered. Identifying the cause of the potential infection in a nursing home resident can be troublesome. These factors may contribute to the high use of antibiotics in nursing homes and must be factored into efforts for reducing unnecessary antibiotic use (Corsonello et al., 2015; High et al., 2009; Nicolle, 2014).

\subsection{Antibiotics in Nursing Homes}

Global consumption of antibiotics grew by 39\% between 2000 and 2015 (Friedrich, 2018), with nursing homes contributing highly to this increase (Klein et al., 2018). Antibiotics are the most commonly prescribed medication class in nursing homes. Approximately $40 \%$ of medications 
prescribed in nursing homes are antimicrobials and antibiotics. ${ }^{1}$ The most frequently prescribed antibiotic in Ontario nursing homes is nitrofurantoin, accounting for $15 \%$ of antibiotics prescribed in nursing homes (Daneman et al., 2011). Other highly utilized antibiotics in nursing homes include trimethoprim/sulfamethoxazole and ciprofloxacin (Nicolle et al., 2005). It is estimated that $50-75 \%$ of antibiotics prescribed in nursing homes are considered potentially inappropriate and should not have been prescribed (Nicolle, 2000; Peron et al., 2013).

Polypharmacy, or chronic intake of multiple medications by older adults, is common and often surpasses 20 medications per person (Sergi et al., 2011; Medeiros et al., 2011). Ramage-Morin (2009) found that almost all nursing home residents were taking prescription medication; 97\% had taken some form of medication within the previous two days and 53\% of residents were found to be taking multiple medications (defined as taking five or more medications within two days). Older adults utilize more medication than any other segment of the population (NCHS, 1986), and nursing home residents represent the highest medication users of older adult populations (Fulton \& Riley Allen, 2005). Between 47-79\% of nursing home residents receive at least one antibiotic course every year, much of which is considered overprescribed (van Buul et al., 2012). Excessive prescription medication, particularly antibiotics, is evidently abundant in nursing homes. The concern is how such excessive medication affects health outcomes (Nicolle, 2000).

\subsection{Adverse Drug Events and Reactions in Nursing Homes}

High antibiotic use in nursing homes increases a resident's risk of experiencing ADEs (Nicolle, 2000). ADEs are preventable and nonpreventable events due to medication consumption. Adverse drug reactions (ADRs) are nonpreventable reactions to medications and are not due to administration error (Field et al, 2001). ADRs are the most serious concern with inappropriate antibiotic prescriptions; a patient's risk of experiencing ADRs increases significantly with excessive antibiotic intake (Nolan \& O'Malley, 1998). ADE incidence in nursing homes is high (1.89 ADEs/1000 resident-months), half of which are considered preventable (Gurwitz et al., 2000). Field et al. (2001) sought to identify predictive factors of high-risk residents for ADEs. High medication intake, particularly antibiotics and psychoactive drugs, was found to play a significant role in increasing a patient's risk for antibiotic-related harm (additional days of

\footnotetext{
${ }^{1}$ For the purpose of this thesis, the terms 'antibiotics' and 'antimicrobials' will be used interchangeably.
} 
antibiotics accounting for $0.4 \%$ increase in risk). In an effort to understand and prevent ADEs in nursing homes, Gurwitz et al. (2000) conducted a cohort study of 18 nursing homes in Massachusetts and found that most preventable ADEs were severe and life-threatening - a finding dissimilar to other clinical settings - and usually occurred during the ordering and monitoring stages of prescribing. Prevention of ADEs is a critical matter in nursing homes and requires examination and limitation of antibiotic use; however, defining antibiotic inappropriateness is a challenging and controversial patient-specific task (Gurwitz et al., 1990).

\section{Inappropriate Antibiotics in Nursing Homes}

Much antibiotic use in nursing home settings has been referred to as inappropriate; however, defining antibiotic inappropriateness is a challenging endeavor. The process of prescribing antibiotics involves several steps and each have the potential to be conducted inappropriately. Inappropriate prescriptions may refer to overuse, misuse, and underuse of antibiotics (Dyar et al., 2015). Additionally, the dose of an antibiotic, the duration of the treatment plan, and the route of antibiotic administration can be prescribed inappropriately (Barlam et al., 2016). Inappropriate antibiotic prescriptions have many causes: lack of diagnostic tools, communication difficulties, staff turnover, resident/advocate pressure, provider prescribing tendencies, infection prevention and control programs (IPAC) resources, and transition of care (Figure 1).

A common basis for prescribing antibiotics is responding to a resident's symptoms or signs. To accurately discern the cause of a resident's symptoms requires the appropriate use of diagnostic testing (Ilić et al., 2015). However, nursing homes often lack diagnostic tools and diagnostic testing is frequently used improperly in nursing homes (Katz et al., 1990; Loeb, 2000). The lack of diagnostic tools is related to the prevalence of empiric prescribing in nursing homes.

Antibiotics in nursing homes are commonly prescribed empirically, meaning prescribing usually broad-spectrum antibiotics without clinical indication of infection nor microbiological evidence (van Buul et al., 2012). Although empiric antibiotic therapy is not necessarily inappropriate, as it is a mostly effective method for initial treatment, its use is due to the lack of biomarkers and diagnostic testing, which is a driver for the spread of AMR. Thus, improved access to diagnostic tools and biomarkers will reduce the need for empiric prescribing, prevent excessive use of 
broad-spectrum antibiotics, and may prevent the spread of AMR (Crnich et al., 2015; Schwartz et al., 2007).

Certain other antibiotic prescribing practices may be considered inappropriate: prescribing antibiotics without identifying the source of the infection and in the absence of symptoms, and prescribing antibiotics as a prophylaxis (to prevent future infection) under certain circumstances, such as for asymptomatic bacteriuria and Foley catheters; however, this remains disputed (Katz et al., 1990; Forbes \& Withers, 1995; Pickard et al., 2018). Beyond selecting an appropriate antibiotic, factors such as dose and duration need to be considered carefully. Incorrect doses of antibiotics and longer than necessary treatment durations are both common prescribing practices considered inappropriate (van Buul et al., 2012). Additionally, antibiotic utilization and inappropriateness is not universal to all nursing homes. Antibiotic use is facility-dependent; antibiotics are utilized more in some facilities than others. Improving antibiotic prescriptions requires identifying high-use nursing homes for interventions (Daneman, 2015). 


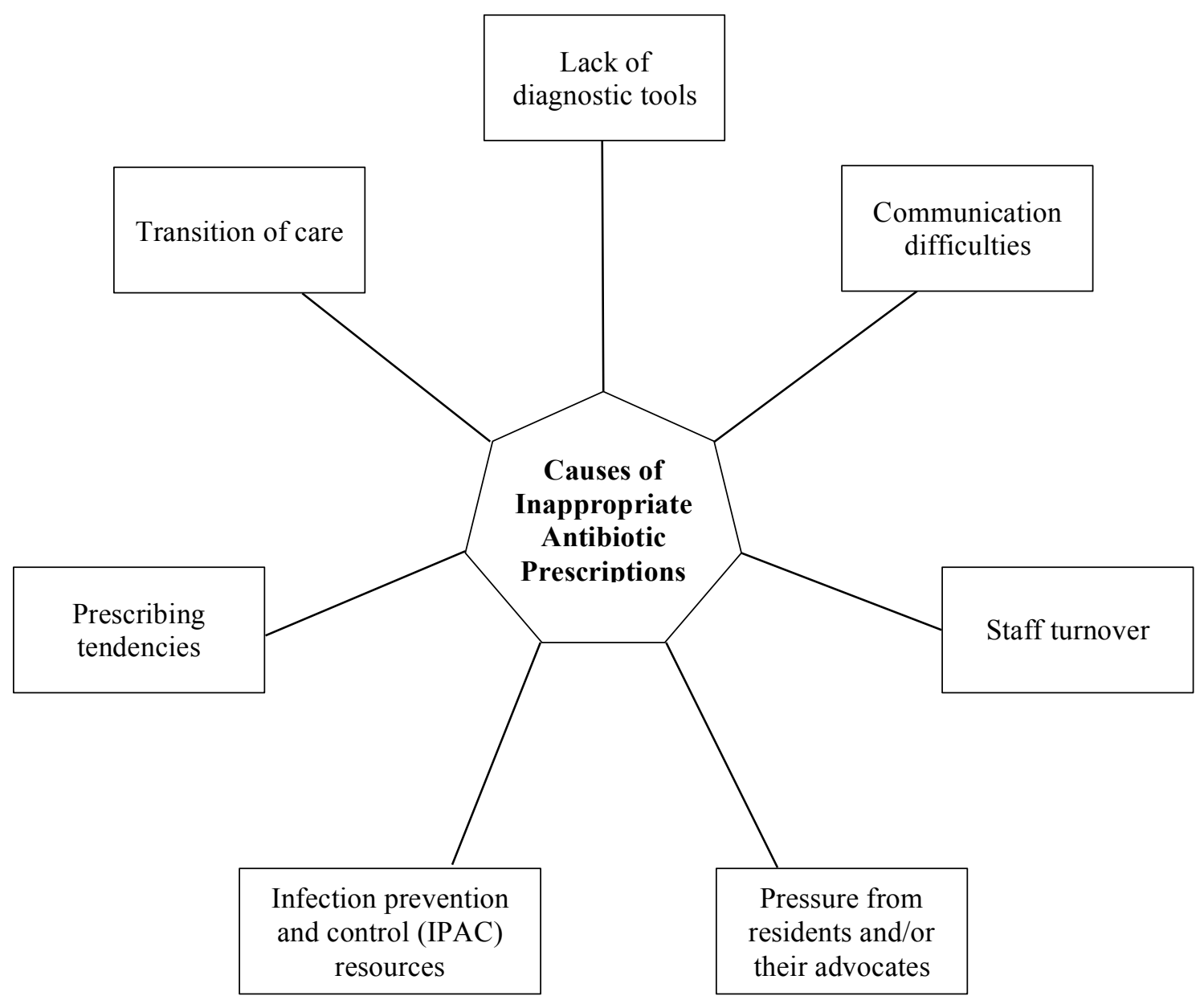

Figure 1. Causes of Inappropriate Antibiotic Prescriptions in Nursing Homes

\subsection{Nursing Home Variation}

Daneman et al. (2011) examined the use and variability of antibiotics across multiple Ontario nursing homes. Facilities were divided into quintiles depending on their magnitude of antibiotic prescriptions. There was a five-fold difference in prescription prevalence between the highest and lowest facility quintiles; meaning, antibiotic use is highly variable between nursing home facilities. This finding supports a previous study, which found significant and substantial interfacility variability in prescriptions of antimicrobials (Benoit et al., 2008). The cause of such variability, however, is unclear. Previous studies have found differences in infection rates as the cause for 50\% of antimicrobial prescription variability (Mylotte, 1999). Conversely, others have argued that resident characteristics are not the cause of the variability; rather, prescriber differences are the cause for such variability (Daneman et al., 2015). In Benoit et al.'s study (2008), facilities with skilled nursing and medical care exhibited higher rates of appropriate 
antibiotic prescriptions than those without. The significance of identifying facilities with high antibiotic consumption is that residents of high-use nursing homes are $24 \%$ more likely to experience antibiotic-related adverse events, such as emergency department visits and hospitalizations as a result of CDI. Identifying the characteristics that cause a nursing home to utilize more antibiotics is a critical component for improving antibiotic use, as high use nursing homes can specifically be targeted for antibiotic improvement (Daneman et al., 2015).

\subsection{Nursing Home Characteristics}

Nursing home characteristics can play a major role in the extent of antibiotic consumption. It has already been mentioned that nursing homes vary substantially in their use of antibiotics. A study of over 600 nursing homes in Ontario, Canada, found a 10-fold difference between the lowest and highest antibiotic-use nursing homes. Two nursing home characteristics were predictive of inappropriate antibiotic use: (1) size of the facility, where larger facilities were tied to more adverse events; and (2) the location of the facility, where rural homes were less likely to experience antibiotic-related harms (Daneman et al., 2015).

Carter et al. (2017) examined perceptions of antibiotic use in nursing homes. Facility culture was found to be a significant factor for nursing homes that used antibiotics more appropriately. Facility culture was defined as interconnectedness among providers, nurses, resident advocates, and other staff. Facilities with better antibiotic use were more likely to exhibit better practice patterns, be more proactive in their infection control programs, have better communication between staff, and show an overall greater level of interconnectedness. Several nursing home characteristics may contribute to the inappropriate use of antibiotics in nursing homes: nursing staff; provider prescribing tendencies; residents and their advocates (including families); infection, prevention and control resources; difficulty diagnosing infections; interdisciplinary communication; and transition of care (Dyar et al., 2015).

\subsubsection{Nursing Staff}

Nursing staff are often the driving force for antibiotic prescriptions in nursing homes. Nursing homes tend to lack on-site diagnostic equipment and often wait for blood test and urine culture results. Pressure is then placed on nursing staff to treat patients quickly and effectively. Such pressure, however, often results in unnecessary antibiotic prescriptions (Dyar et al., 2015; Crnich 
et al., 2015). Nursing homes often have many providers apportioning care. Often, prescribers are off-site and visit the facility occasionally (Lam et al., 2012; Sloane et al., 2002). Nursing staff are thus required to provide over-the-phone descriptions of patient symptoms to the off-site physicians. Communicating symptoms over the phone is a challenging task. If symptoms are not described sufficiently or accurately, a prescriber may recommend an antibiotic, even though one may not be required or necessary. Few resources in nursing homes are provided for improving nursing staff communication skills. If inappropriate antibiotics due to ineffective communication are to improve, nursing staff must be provided training to ensure patient symptoms are communicated correctly (Squillace et al., 2009; Warren et al., 1991).

An additional concern in nursing homes is the high rate of nursing staff turnover. High turnover rates in nursing homes are tied to decreased job satisfaction and increased workload for other staff (O’Brien-Pallas et al., 2008). Turnover rates are also linked to disturbances in the continuity of care, and increased medical errors and length of stay in a facility. Such increased medical errors may contribute to inappropriate antibiotic prescriptions (Staw, 1980; Robertson et al., 1994; O’Brien-Pallas et al., 2008). Ensuring quality of care for residents in nursing homes requires optimal communication between nursing staff levels, including registered practical nurses (RPNs) and registered nurses (RNs) (Castle \& Engberg, 2005).

\subsubsection{Provider Prescribing Tendencies}

An important cause of inappropriate antibiotics in nursing homes is provider prescribing tendencies. Daneman et al. (2017) conducted a population-based study of provider characteristics. The purpose of the study was to assess such characteristics related to three prescribing tendencies: (1) the initiation of antibiotics, (2) prescribing excessively long therapy durations, and (3) selecting inappropriate broad-spectrum antibiotics (such as fluoroquinolones). Historical tendencies were found to be predictive of future prescribing patterns. Providers who previously were more likely to initiate antibiotic treatment were more likely to initiate antibiotic treatment in the future, and the same would apply for prescribing excessively long therapy durations and selecting for inappropriate antibiotics. These findings support a previous study's conclusions that provider characteristics were more predictive of prolonged therapy durations than resident characteristics (Daneman et al., 2013). 


\subsubsection{Residents and Advocates}

Another important nursing home characteristic that influences prescriptions of inappropriate antibiotics is resident and advocate expectations. Residents and advocates typically have a rudimentary understanding about appropriate antibiotic prescribing; they tend to believe that antibiotics are necessary when they are not (Dyar et al., 2015). This pressure on prescribers is one of the common causes of inappropriate antibiotic prescriptions in nursing homes. Residents and their advocates need to be aware of the intricacies of diagnosing a true infection and the dangers of inappropriate antibiotic prescriptions (Mitchell, 1982; Sloane et al., 2016).

\subsubsection{Infection, Prevention, and Control Resources}

In comparison to hospitals, nursing homes tend to lack infection prevention resources. IPACs are components of health care institutions ensuring vulnerable individuals are protected against infections (World Health Organization (WHO), 2015). IPACs in hospitals have been effective for significantly reducing rates of urinary tract infections (UTIs), pneumonia, and surgical infections (Haley et al., 1985). A review of IPAC practices in Australian nursing homes found that although most had designated IPAC personnel, only a fraction were certified and trained adequately (Stuart et al., 2015). Hand sanitization is a cornerstone for IPACs; yet, in a review of hand hygiene in nursing homes, hand washing only occurred $27 \%$ and $63 \%$ of the time before and after interacting with patients (Thompson et al., 1997). IPACs are critical to nursing homes; however, IPAC professionals often have competing responsibilities, such as wound care and education. As such, adherence of nursing staff to IPAC principles is variable and tends to be low. Improved communication between nursing staff and IPAC specialists is critical for better adherence to IPAC guidelines (Matheï et al., 2007; Mody et al., 2005; Mody et al., 2010).

\subsubsection{Difficulty Diagnosing Infections}

Diagnosing infection is a troublesome task even for the most experienced physicians (Nicolle, 2000). As mentioned earlier, empiric therapy is common in nursing homes and its prevalence is linked to the lack of on-site laboratories, difficulty determining infections, and lacking resident history (Benoit et al., 2008). Other factors contributing to the difficulty of diagnosing infections in nursing homes include communication barriers, chronic symptoms, comorbidities (Genao \& Buhr, 2012), and the presence of multiple infections or diseases (Valderas et al., 2009). Often the 
cause of a patient's symptoms is ambiguous; it can be due to an infection, another drug, or some unknown source. Such ambiguity acts as a driving force for antibiotic prescriptions. Physicians often prefer to prescribe an antibiotic, even when there is no indication, to ensure that any potential infection is treated. Such "just in case" prescribing behaviours need to be addressed to reduce inappropriate antibiotic consumption (Sakuma et al., 2011; Sloane et al., 2016).

\subsubsection{Urinary Tract Infections}

Urinary tract infections (UTIs) are the most common infection in nursing homes. An estimated $30-50 \%$ of antibiotics prescribed in nursing homes are for treating UTIs; particularly, asymptomatic UTIs. One of the most common examples of inappropriate antibiotic prescribing in nursing homes is for the treatment of asymptomatic UTIs, where bacteria is present in the urine but without displayed symptoms (Nicolle et al., 1996; Loeb et al., 2002; Nicolle, 1997; Nicolle, 2000). Unlike symptomatic UTIs, asymptomatic UTIs do not benefit from antibiotic therapy. Antibiotic treatment of asymptomatic UTIs is linked to increased mortality, adverse events and reinfection with resistant bacteriuria (Ouslander et al., 1996; Abrutyn et al., 1994; Nicolle et al., 1983; Nicolle et al., 1987). Although many studies have demonstrated the inappropriateness of antibiotic treatment of asymptomatic UTIs, it continues to be one of the most common causes for antibiotic prescriptions in nursing homes (Katz et al., 1990; Pickering et al., 1994). Without standardized assessment of potential UTIs, it can be highly challenging for health care professionals to recognize acute symptoms, due to communication barriers (such as hearing impairment, language disorders such as aphasia, and dementia), and accurately distinguish a symptomatic and asymptomatic UTI (Nicolle, 1999).

In March 2000, the Society for Healthcare Epidemiology of America (SHEA) developed guidelines and established minimum criteria for initiating antibiotics for suspected infections in nursing homes; however, guidelines for specific antibiotics were not considered (Nicolle et al., 1996). In an effort to establish minimum criteria for initiating antibiotics, Loeb et al. (2001) utilized a Modified Delphi panel: a tool for arriving at consensus via recruitment of an expert panel (Jones \& Hunter, 1995). Minimum criteria for initiating antibiotics specific to suspected UTIs were established for residents with and without an indwelling urinary catheter (IUC). For residents without an IUC, minimum criteria included dysuria (painful urination) alone or a fever $\left(>37.9^{\circ} \mathrm{C}\right)$ and any of the following: sudden or worsening urgency, frequency, suprapubic pain, hematuria, tenderness, or incontinence. For residents with an IUC, minimum criteria included 
any of the following: fever, tenderness, rigors, or delirium (Loeb et al., 2001). In 2005, Loeb et al.'s criteria for assessing UTIs were modified to include nursing-home specific guidelines for treating UTIs (Loeb et al., 2005). Since then, nursing home adoption of the modified Loeb criteria is recommended and has been successfully implemented in some institutions; yet, antibiotic treatment of asymptomatic UTIs remains high for many nursing home facilities (Genao \& Buhr, 2012; Loeb et al., 2005).

\subsubsection{Communication Difficulties}

Prescriber and nursing staff communication is an important cause of inappropriate antibiotic prescribing in nursing homes. Communication between staff can significantly influence attempts at improving antibiotic prescribing (European Surveillance of Antimicrobial Consumption, 2009). Nursing homes staff across Canada are licensed and unlicensed. Licensed nursing staff include RNs, RPNs, and licensed practical nurses (LPNs). Nursing homes often have unregulated health care providers and aides. Licensed nurses are generally responsible for assessing residents and providing nursing care, whereas, aides are largely more responsible for assisting with activities of daily living (Najib Ayas, 2010).

As previously mentioned, nursing staff are recognized as the front line cause of antibiotic prescriptions, due to call-in orders from physicians, rather than physician bedside visits (Zabarsky et al., 2008; Daneman et al., 2011). Nurses often lack training for accurate evaluation of potential bacterial infection, such as for dipstick urinalysis (Lim et al., 2014). Efforts for improving knowledge through education can be hindered by the high rate of staff turnover in nursing homes (Mylotte, 1996). Off-site physician prescriptions account for approximately 50\% of medication orders in nursing homes (Gurwitz et al., 1990). Thus, physicians often lack resident-related knowledge, such as information that can be pertinent to the appropriateness of subsequent antibiotic prescriptions (Barber et al., 2009). These physicians may select broadspectrum antibiotics, antibiotics that are effective against multiple bacterial infections (Levy, 1998), for longer durations than necessary to ensure successful therapy (Charani et al., 2010). Excessive use of broad-spectrum antibiotics is an inadvisable practice, as it can increase the risk of disrupting a patient's microbiome and acquiring resistant bacteria (Levy, 1998). Improved communication between different levels of nursing staff and prescribers is essential for better antibiotic utilization. 


\subsubsection{Transition of Care}

The transfer of nursing home residents between nursing homes and acute care hospitals plays a role in the cause of antibiotic inappropriateness and adverse outcomes. Nursing home residents who visit emergency departments (ED) often receive antibiotic prescriptions that are unnecessary, which is a challenging factor for prescribers to control (Morrill et al., 2016). Beyond antibiotic prescriptions, ED visits place nursing home residents with increased risk of acquiring antibiotic resistant bacteria and CDI. Residents acquiring antibiotic-resistant bacteria are becoming increasingly common in nursing homes (Campbell et al., 2009). One particular cause for such increased prevalence is inappropriate treatment of suspected UTIs with antibiotics (Rotjanapan et al., 2011); however, a frequent pathway for residents acquiring antibiotic resistant organisms is via transfer between acute care and nursing homes (Bonomo, 2000). Up to one-third of nursing home residents are transferred to EDs per year; however, there is substantial variability across homes. Factors that increase the likelihood of ED transfer include proximity of nursing homes to EDs, location of facility (urban vs. rural), size of facility (transfer is more likely in larger nursing home facilities), and previous high rates of ED transfers (Gruneir et al., 2016). Considering the high flow of residents between nursing homes and acute care (Kahvecioglu et al., 2014), preventing unnecessary antibiotic prescriptions requires improving prescribing in acute care and communication between acute care and nursing homes (Morrill et al., 2016). Improving antibiotic prescriptions in nursing homes is a difficult task that requires examining every aspect of nursing home care and the barriers that are preventative (Crnich et al., 2015; Lim et al., 2014; van Dijk et al., 2005; Smith et al., 2008; Shield et al., 2005; Katz et al.; 1990; \& Squillace et al., 2009). 


\section{Improving Antibiotic Use}

Antibiotic use during the 1900 s resulted in drastically improved patient care. However, with such increased use, the question of inappropriate use surfaced (Reimann, 1961). Effects of inappropriate antibiotic use gained attention, such as increased economic costs and the spread of AMR. In the earlier days of AMR, new antibiotics were in development and were capable of controlling the spread of resistance; however, recent antibiotic development has slowed and cannot reliably and indefinitely manage the spread of AMR (Spellberg et al., 2008). Improving antibiotic use has been recognized by the WHO as vital to prevent the spread of AMR, which is now recognized as a major threat to human health (So et al., 2010; Bartlett, 2011).

\subsection{Antimicrobial Stewardship Programs (ASPs)}

Antimicrobial stewardship programs (ASPs) are health care initiatives and interventions to optimize the use of antimicrobials (including antibiotics), whilst minimizing the occurrence of ADEs, costs of therapy, and preventing the spread of AMR. ${ }^{1}$ ASPs accomplish this task by ensuring appropriate antibiotics are selected with optimal dosage, route of administration, and duration of therapy (Dellit et al., 2007). It is estimated that with immediate implementation of ASPs, 619,000 cases of CDI can be prevented in the US over a 5-period period, and the overall spread of antibiotic resistance can be reduced (Slayton et al., 2015; Kaki R, 2011). ASPs in acute hospital care have demonstrated a capacity to improve antibiotic use, costs, ADEs and resistance, without negatively impacting clinical outcomes (Kaki et al, 2011).

Nowak et al. (2012) investigated ASPs requiring physicians to review summary reports of antibiotic use for adult inpatients. Antibiotic expenditure was growing yearly by $14.4 \%$ prior to implementation of the intervention but decreased by $9.75 \%$ following implementation, a finding that was sustained for subsequent years, providing approximately $\$ 1.7$ million in savings. Although many studies can attest to the necessity of ASPs, not all implemented ASPs are successful. Success of an ASP is highly dependent on the design of the program and the staff that are targeted (Dellit et al., 2007; Grol \& Grimshaw, 2003; Sloane et al., 2016). The IDSA (Barlam et al., 2016), SHEA (Loeb et al., 2001) and CDC (CDC, 2015) have recommended the implementation of ASPs in all health care institutions. However, most ASP studies have focused on acute care hospitals and ambulatory settings. Several systematic reviews have been conducted

\footnotetext{
${ }^{1}$ For the purpose of this paper, the terms 'ASP' and 'intervention' will be used interchangeably.
} 
for ASPs in community, assisted living, hospital, and nursing home settings; few, however, have thoroughly reviewed nursing homes, and systematic reviews that have investigated ASPs in nursing homes have indicated heterogeneous results (Sloane et al., 2016).

\subsubsection{ASPs in Nursing Homes}

In 2015, the CDC published seven core elements of ASPs in nursing homes: (1) facility leadership commitment, (2) accountability, (3) drug expertise, (4) actions for improving antimicrobial use, (5) education, (6) tracking of antimicrobials, and (7) reporting of antimicrobials. Nursing home settings ought to be prime candidates for ASPs. Inappropriate use of antibiotics is prevalent in nursing homes and is linked to the vulnerability of residents to ADEs and the increased prevalence of antibiotic resistant infections. However, literature describing ASPs in nursing homes remains relatively limited (Strausbaugh \& Joseph, 2000; Hughes, 2011; Jump et al., 2012). Being a nursing home resident is one of the most important risk factors for MDRO colonization (Cassone \& Mody, 2015). Nursing home residents are highly susceptible to infection, especially due to comorbidity, cognitive deficits, and use of invasive devices. Thus, nursing home residents would greatly benefit from programs implemented to ensure their care is optimized (Martin, 2017).

Although hospitals and nursing homes have a degree of overlap, extrapolating the effectiveness of hospital ASPs to nursing homes is complicated. ASP outcomes in hospitals may not be generalizable to nursing homes due to several factors: differences in length of stay and age of patients/residents, cognitive impairments (such as Alzheimer's), availability of on-site physicians, roles of nurses and resident advocates for decision-making, lack of resources in nursing homes, and differences in prescribing guidelines. Thus, evidence supporting ASPs need to be specific to nursing homes (Sloane et al., 2016).

\subsection{Designing ASPs}

In 1997, the IDSA published guidelines for ASPs in health care settings and recommendations for best practice. To update the ASP guidelines, the IDSA published a revised set of recommendations for implementing successful ASPs. A large component of this effort included organizing a core ASP team for the facility with a variety of positions: an infectious disease (ID) physician, clinical pharmacists, a clinical microbiologist, an information system specialist, an 
infection control professional, and a hospital epidemiologist. Other components of successful ASPs included collaboration between the ASP and IPAC teams, and obtaining administrative support and leadership (Dellit et al., 2007).

Designing ASPs requires addressing all areas of the antibiotic prescribing process. ASPs need to detect changes in antibiotic use, focus on areas of high antibiotic use, and measure outcomes related to observed changes through risk adjustment and development of benchmarks (Sloane et al., 2016; Ibrahim \& Polk, 2014). Incorporation of multilevel collaboration is significant for implementing effective ASPs; however, ASPs are unlikely to be successful without an underlying framework that provides guidance for implementation. As altering prescribing behaviour is the fundamental goal of ASPs, behaviour modification must be central for any intervention to be successful. ASPs that are purely guideline-focused are unlikely to significantly modify behaviour. Existing guidelines are vast and widely available; yet, most are ineffective for lasting behaviour change (Thaler \& Sunstein, 2008; Charani et al., 2010). Thus, guidelines need to focus on behaviour modification; a system for categorizing interventions by likelihood of effectively altering behaviours may be a necessary venture for designing ASPs (Michie et al., 2011; Schuster et al., 1998).

\subsubsection{Barriers and Facilitators}

Although a successful ASP has the capability of improving care for patients in health care institutions, there remain many obstacles in the way of designing and implementing an effective ASP that need to be considered (Dellit et al., 2007; Grol \& Grimshaw, 2003; Charani et al., 2010; Malani et al., 2016; Colón-Emeric et al., 2016). Successfully designing ASPs requires overcoming barriers at every level of health care including the individual, the team, and the organization; strategies for improving antibiotic use need to be tailored to these specific levels (Ferlie \& Shortell, 2001). A survey investigating ASPs implemented into 86 nursing homes in Michigan found significant barriers impeding the effectiveness of ASPs. Although interest in implementation of ASPs is high, nursing home staff may not know how to select and implement an ASP (54\%), do not have the knowledge to propose ASPs (50\%), and are constrained and cannot prioritize strategies for implementing ASPs (8\%) (Malani et al, 2016).

A whole-system approach is critical towards implementing successful ASPs. Incorporation of ASPs into other quality improvement initiatives and infection control programs, such as hand 
hygiene initiatives, can significantly improve its effectiveness (Crnich et al, 2015; Baur et al., 2017). Simple ASPs, such as guideline-focused ASPs, are likely not capable of significantly altering prescriber behaviour (Charani et al., 2010). There are many instances where patients' conditions are too complex for solely guideline-focused change (Schuster et al., 1998). Most guidelines are designed for addressing individual diseases but patients with comorbid conditions can have multiple diseases. Such patients may experience ADEs due to drug-disease interactions (Boyd et al., 2005). Thus, efforts to address the multitude of factors relating to inappropriate antibiotic prescriptions may require complex interventions (Dolan et al., 2010).

\subsubsection{ASP Complexity}

Complex ASPs are generally multifaceted educational interventions targeted at multiple staffing levels (Colón-Emeric et al., 2016). There is evidence to suggest that complex interventions are necessary to produce lasting prescribing changes. A physician's tendency to prescribe antibiotics is rooted in cognitive and behavioural factors. ASPs are, fundamentally, efforts to implement and improve evidence-based practice. Doing so requires behavioural change. Designing ASPs requires understanding behaviour from a theoretical framework and applying behaviour change techniques (Dolan et al., 2010; Michie et al., 2013). There is sufficient evidence to suggest that including feedback and action planning in an intervention is necessary to ensure behavioural change (Davey et al., 2015). However, some studies have found limitations regarding complex interventions. Complex interventions may lack sustainability, as they require extensive resourcing (Moullin et al., 2015; Colón-Emeric et al., 2016). Indeed, feedback is a necessity for behavioural change techniques; however, providing feedback for a complex intervention with many data elements can be challenging (Davey et al., 2015). Studies have reported that complex interventions are often discontinued in the post-intervention phase but these finding are not conclusive (Bond et al., 2014; Stirman et al., 2012).

Some of the success of a complex intervention is dependent on the perception of its effectiveness. If an intervention is understood to be effective for improving patient outcomes, motivation to modify behaviour will be higher (Colón-Emeric et al., 2016). Perception of an intervention's complexity is dependent on staff; some staff may find an intervention too simplistic while others may find it overwhelming. As mentioned earlier, front-line staff play a crucial role in the prescribing of antibiotics (Zabarsky et al., 2008). ASPs need to promote a 
culture of shared knowledge and open interdisciplinary communication; doing so will ensure front line staff and other health care providers (HCPs) are aware of the dangers of inappropriate antibiotics and, thus, will be more motivated for improvement (Charani et al., 2010).

The means of delivering ASPs is a significant factor influencing its success. Group sessions allow for learning through dialogue and conversation; however, one-on-one sessions allow for customizability and clarification (Colón-Emeric et al., 2016). Thus, ASPs need to have balanced complexity, delivered in a manner that is well received, and tailored to specific staffing levels (Colón-Emeric et al., 2016; Thaler \& Sunstein, 2008).

\subsection{Efforts to Organize Interventions}

Interventions for administering ASPs are vast and diverse, and can be customized to fit many types of facilities (Septimus \& Owens, 2011). Intervention objectives can be generally divided into two categories: patient level recommended care, such as adherence to guidelines, and strategies for accomplishing objectives, such as through antibiotic restriction (Davey et al., 2013).

\subsubsection{IDSA's Stewardship Guidelines}

In 1997, the IDSA and SHEA emphasized the need for ASPs to prevent AMR in hospitals (Shlaes et al., 1997). In 2012, the IDSA and SHEA recruited a panel consisting of 18 interdisciplinary experts to organize and provide recommendations for ASP implementation in inpatient populations, including long-term care. The expert panel was divided into five subgroups to assess and provide recommendations for implementation of ASPs: (1) interventions, (2) optimizing antibiotic administration, (3) microbiology and laboratory diagnostics, (4) measurement and analysis, and (5) ASP for special populations (Barlam et al., 2016). ASPs were evaluated using the Grading of Recommendations Assessment, Development and Evaluation (GRADE) System (Figure 2).

The GRADE system is a tool for assessing guidelines that consists of three components: (1) rating the quality of the evidence, (2) determining the strength of the recommendation, and (3) the implications of the strength of the recommendation. Determining the quality of evidence involves a 3-step process. An initial confidence level is established; study designs such as randomized controlled trials (RCTs) provide a high initial confidence level, whereas 
observational studies provide a low initial confidence level. The confidence level is then either raised or lowered depending on several factors: risk of bias, inconsistency, effect size, etc. A final evaluation of the overall confidence level is determined. The overall quality of the evidence is determined based on the final level of confidence and is assigned as high, moderate, low, or very low.

To determine the strength of the recommendation, four factors are considered: (1) the quality of the evidence, (2) the balance of benefits and harms, (3) patient values, and (4) resources. Items are then categorized as a strong or weak recommendation. A strong recommendation means that most individuals involved with the program would desire its implementation, health care workers believe most people ought to receive such a program, and such a program can be implemented as policy under most circumstances. A weak recommendation differs in that many individuals involved with a program would desire its implementation but many would not, health care workers are inconsistent with their beliefs about the program, and much debate would be needed prior to incorporating the program into policy (Atkins et al., 2004; US GRADE Network, 2015).

The GRADE system has been incorporated into many organizations, including the WHO, the National Institution of Clinical Excellence (NICE), the Cochrane Collaboration, the Ministry of Health and Long-Term Care (MOHLTC). Many other international organizations have also implemented the GRADE system (Alonso-Coello et al., 2013; Schünemann et al., 2006). An emphasis in the development of the GRADE system was to distinguish between the strength of a guideline and the underlying quality of the studies; however, GRADE itself does not have evidence supporting its reliability nor validity. Thus, determining the validity of the IDSA's ASP guidelines may be difficult (Kavanagh, 2009). 


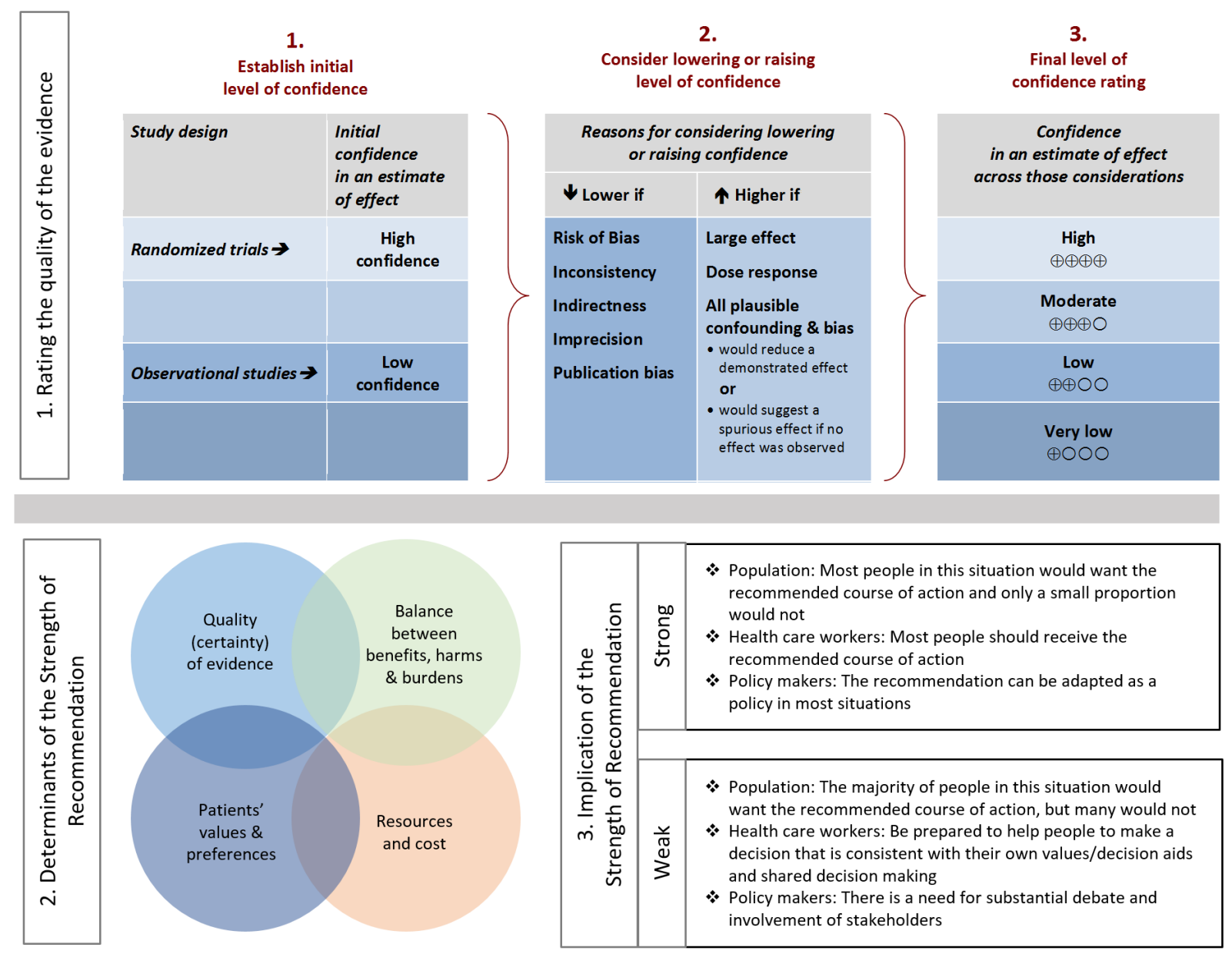

Figure 2. The GRADE System for rating quality of evidence and determining the strength of recommendations.

Reproduced with permission from the U.S. GRADE NETWORK.

\subsubsection{Public Health Ontario's ASP Strategies}

In 2016, Public Health Ontario (PHO) identified and listed 32 ASP strategies (including tools, interventions, and activities) for implementation in health care institutions (primarily hospitals) to improve antimicrobial use and educate prescribers. To aid health care institutions in their selection of ASPs, PHO evaluated each intervention for its priority, difficulty, and evidence to support specific outcomes. PHO's ASP team, consisting of pharmacists and physicians, determined the priority rating for each intervention independently. 
Five assessment criteria were used to determine the priority of the ASP: (1) importance (if the ASP was identified as fundamental), (2) if the ASP was identified as fundamental in the literature or by national organizations, (3) evidence supporting outcomes, (4) if the ASP was suggested by Accreditation Canada, and (5) the adaptability of the ASP to institutions of various sizes and resources. Priority ratings were given as A, B, or C (with A as highest priority and C as lowest). To determine the difficulty rating, ease of implementation and sustainability of the ASPs were evaluated independently. Difficulty ratings were scored using a 1-3 level system (where 1 was least difficult and 3 most difficult). Systematic reviews were used to assess the evidence supporting ASP outcomes for each strategy. Outcomes were organized into five categories: (1) drug utilization, (2) prescribing behaviour, (3) clinical outcomes, (4) reduction of CDI, and (5) reduction of other resistant infections. PHO then organized the ASPs into a list of "PHO Core Strategies"; these were strategies that were determined to be foundations to institutional ASPs. Six interventions were listed as Core Strategies: (1) empiric antibiotic prescribing guidelines, (2) formulary automatic substitution/therapeutic interchange policies, (3) formulary restriction, (4) formulary review/streamlining, (5) intravenous to oral conversion, and (6) prescriber education.

There are several limitations to PHO's list of ASP strategies. Through examination of systematic reviews, it was determined that the overall quality of evidence is low, especially for clinical outcomes. The effectiveness of ASPs is dependent on the outcome of interest and the extent of its effect varies between studies. Certain studies reviewed were particular to specific antibiotics, infections, and health care settings; this leaves unknown the generalizability of such studies to other antibiotics, infections, and facilities such as nursing homes. Additionally, many studies utilized a multifaceted technique that incorporates many interventions. Determining the effect of individual components is difficult. Lastly, the sustainability of the interventions was not sufficiently reviewed (PHO, 2016).

\subsection{Interventions}

Identifying and contrasting ASP interventions was a central component of this thesis. Fourteen interventions (and a later additional intervention) were identified, including PHO's Core Strategies, ASPs from IDSA's stewardship guidelines, and those identified from systematic reviews: antibiograms, antibiotic cycling, audit and feedback, clinical decision support systems, de-escalation, educational interventions, formulary automatic substitution, formulary restriction, 
formulary review, guidelines for empiric prescribing, intravenous to oral transition, multifaceted interventions, short course antibiotic therapy, and targeting CDI. Each will be discussed in this section. (Barlam et al., 2016; Baur et al., 2017; Katz et al., 2017; Marshall et al., 2017; Nicolle, 2014; Fleming et al., 2013; Tonkin-Crine et al., 2017; Losier et al., 2017; Arnold \& Sharon, 2005).

\subsubsection{Antibiograms}

One of the most common barriers to implementing ASPs in nursing homes is the lack of resources (Nicolle, 2000). An important resource that most nursing homes lack is standardized antibiograms (Kullar et al., 2017). Antibiograms are a tool for summarizing local susceptibility of bacteria to antibiotics at a given point in time, easing the process of appropriately selecting and prescribing antibiotics (Joshi, 2010). Antibiograms provide prescribers with facility-specific bacterial resistance data and guidance for empirically prescribing appropriate antibiotics. Antibiograms have been demonstrated to be effective for reducing inappropriate antibiotics in acute-care settings; yet, their value in nursing homes remains mostly unknown (Furuno et al., 2014; Morrill et al., 2016).

Development of antibiograms is a recommended component of the Microbiology and Laboratory Diagnostics category of the IDSA's ASP guidelines; however, the intervention received a weak recommendation and the evidence supporting such a strategy is low (Barlam et al., 2016). A survey of ASPs in nursing homes in Michigan found that roughly half had access to antibiogram data (Malani et al., 2016). Overall absence of this tool may be indicative of the lack of resources

present in nursing homes. Developing antibiograms requires access to a microbiology laboratory. Unfortunately, many smaller nursing homes do not have such access; thus, implementation would be resource intensive (Sloane et al., 2016; Chopra \& Rivard, 2015).

Furuno et al. (2014) implemented antibiogram use in a community-based skilled nursing facility. Creation of the antibiograms incorporated clinical culture data over 6 months, admission clinical culture data from an adjacent acute care facility, and medical chart data. Prior to implementation of antibiograms, $85 \%$ of antibiotics were prescribed empirically and only $35 \%$ of antibiotics were prescribed appropriately. Appropriate empirical antibiotic prescribing was defined as residents that had clinical cultures collected and received empirical antibiotics that successfully eradicated the infecting organism. Following antibiogram implementation, appropriate antibiotic 
prescriptions increased to $45 \%$, much of which is likely due to improved empiric prescribing; however, the finding was statistically insignificant. Though the results indicate a potentially effective intervention, few studies supporting antibiograms in nursing homes are available. Antibiograms may only be an effective intervention in combination with other ASP strategies. Further evidence is needed to conclude the practicality of antibiograms (Crnich et al., 2015; Furuno et al., 2014; Morrill et al., 2016).

\subsubsection{Audit and Feedback}

Another ASP intervention that has been studied considerably is the use of audit and feedback and its variations. Audit and feedback is a tool for providing a summary of an individual's clinical performance for a given prescribing behaviour over a specific period, with the purpose of assessment to facilitate adjustment of performance (Jamtvedt et al., 2006). Evidence suggests that prescriber tendencies are the cause for variable antibiotic prescribing patterns between nursing home facilities. Antibiotic prescribing tendencies can be divided into three categories: (1) the tendency to start antibiotics, (2) the tendency to prolong antibiotic therapy, and (3) the tendency to prescribe a specific class of antibiotics, such as fluoroquinolones. Prescribers with greater tendencies to initiate antibiotics, prolong antibiotic therapy, or prescribe inappropriate classes of antibiotics, such as fluoroquinolones, can be targeted for peer comparison audit and feedback interventions (Daneman et al., 2017).

Implementation of audit and feedback requires skilled personnel, which may include clinical pharmacists and ID specialists (Barlam et al., 2016). Audit and feedback is often incorporated into educational interventions and coupled with local resistance data (Schwartz et al., 2007; Barlam et al., 2016). Although audit and feedback is listed as a highly recommended intervention with moderate quality of evidence by the IDSA, the evidence supporting the intervention is mixed (Barlam et al., 2016; Grol \& Grimshaw, 2003). In a systematic review of ASPs in emergency departments, audit and feedback was an effective intervention for improving antibiotic prescriptions for treatment of acute respiratory infections (Tonkin-Crine et al., 2017). Arnold and Straus (2005) systematically reviewed ASPs in ambulatory care and compared audit and feedback (with and without education material) to other interventions, such as educational outreach visits and reminders. Three RCTs were reviewed (Hux et al., 1999; Mainous et al., 2000; O'Connell, 1999); the interventions included explaining prescribing data, prescription comparisons between physicians, and education for optimal antibiotic prescribing. Two of the 
RCTs did not show significant prescribing differences between audit and feedback and other interventions; however, Hux et al. (1999) showed significantly improved prescriptions for firstline antibiotics $(5.1 \% ; P<.01)$ and reduced expenditure by $\$ 3.32$ per physician $(P<.002)$. Evidence suggests that audit and feedback is effective for test orders (blood and culture) and prevention; however, the effect size may be dependent on several factors: the form and source of the feedback provided, the format it is delivered, and the frequency and intensity of the feedback. If possible, audit and feedback interventions should be delivered in combination with education, outreach, and reminders (Grol \& Grimshaw, 2003). Future studies will be needed to demonstrate the applicability of audit and feedback to nursing homes and the most optimal and feasible method for its implementation.

\subsubsection{Clinical Decision Support Systems}

Prescribers are often placed in difficult positions when arriving at a diagnostic decision. Many factors impact a physician's ability to determine an infection, such as communication barriers, comorbid conditions, and patient frailty (Nicolle, 1999; Nicolle, 2014). Clinical decision support systems (CDSS) are interventions that intend to improve the diagnostic decision making process. CDSS can vary in their implementation; however, many are computer automation tools that provide clinicians with relevant information pertaining to a patient's condition and enables for a more reliable diagnosis and selection of appropriate antibiotics (Forrest GN et al., 2014). CDSS can facilitate other ASPs, such as de-escalation, formulary restriction intervention, and audit and feedback (Thursky, 2017).

Corsonello et al. (2015) reviewed ASPs for older patients, particularly educational interventions and computerized decision support. The latter was found to be especially effective for identifying antibiotic related concerns, increasing appropriate prescriptions of antibiotics with inclusion of a clinical pharmacist, and improving empiric antibiotic treatment. However, CDSS are not all universally supported. Although included in the IDSA's stewardship guidelines, CDSS are considered a weak recommendation, though evidence supporting their effectiveness is moderate (Barlam et al., 2016). Additionally, considering the extent of resourcing required to design and apply a novel electronic medical record (EMR) equipped with clinical decision support, implementation of such an intervention would be highly resource-intensive and likely unfeasible. Nursing home implementation of CDSS is relatively unexplored and must be considerate of the cost of such an initiative (Arnold \& Straus, 2005; PHO, 2016). 


\subsubsection{De-escalation}

A common antibiotic prescribing practice previously mentioned is the use of broad-spectrum antibiotics, a class of antibiotics effective for eradicating most infections. Although effective, such practice is sometimes inadvisable, as it promotes the spread of antibiotic resistant bacteria (Levy, 1998). There are times, however, where selection of broad-spectrum antibiotics is reasonable and appropriate. When the infecting organism is not identified and empiric therapy is required, broad-spectrum therapy may be encouraged to ensure that initial therapy is successful (Schwartz et al., 2007). Substituting broad-spectrum antibiotics with narrow-spectrum antibiotics when the infecting organism is identified is essential for preventing resistance; however, studies have shown that such transitions by prescribers are not common even when culture results support the transition (Rotjanapan et al., 2011).

De-escalation interventions target swift transitioning of broad-spectrum to narrow-spectrum antibiotics following completion of culture results. The benefits of transitioning from broadspectrum to narrow-spectrum antibiotics include decreased drug expense and risk of ADEs, and improved health outcomes (Dellit et al., 2007; Schuts et al., 2016). De-escalation interventions have been found to have a profound impact on antibiotic use in hospitals (particularly EDs) for reducing mortality (up to $35 \%$ reduction), but the evidence for its support is heterogeneous between studies. Furthermore, de-escalation interventions remain mostly a neglected area of ASP research in nursing homes. The aforementioned survey of nursing home ASPs in Michigan found that less than half had antimicrobial de-escalation programs. Future studies will be required to conclude the effectiveness of de-escalation interventions in nursing homes (Crnich et al., 2015; Malani et al., 2016; Losier et al., 2007; Pakyz et al., 2015; Schuts et al., 2016).

\subsubsection{Educational Interventions}

Educational interventions have been studied extensively and are often the first-choice intervention for ASPs (Grol \& Grimshaw, 2003; Katz et al., 2017). Educational interventions are common because they are generally feasible and can be complemented with other intervention strategies (Dyar et al., 2015). Educational interventions targeting prescribers and other health care professionals are included as a PHO Core Strategy. An advantage toward utilizing educational interventions is their adaptability to other interventions and their customizability. Educational interventions can have various modifiable components: discussion and education of 
ASP principles and appropriately prescribing antibiotics for specific infections, and engaging in activities that complement ASPs (ordering urine and blood cultures, identifying drug allergies), etc. (PHO, 2016). Education programs can target any level of health care and in numerous settings, and all three domains of prescribing: (1) the patient and their advocates, (2) staff and facility, and (3) health care providers (Crnich et al., 2015). Sessions can be conducted in person or in a group format, whether multidisciplinary (simultaneous sessions including nurses, physicians and other staff) or individualized. An educational intervention can be engaging through interactive discussion, or it can be didactic and provided in a lecture format (Arnold \& Sharon, 2005). An educational intervention can be as simple as sharing educational materials or it can be delivered in combination with other interventions, such as audit and feedback. Although the freedom to select, modify, and customize an educational intervention is an advantage, such freedom can be impeding when implementing an educational intervention in nursing homes. Implementing a successful educational intervention requires careful consideration of the evidence supporting the method and the staff targeted (Charani et al., 2010; Katz et al., 2017).

As previously discussed, nursing staff are often the front line responders to suspected infection and are frequently the only licensed health care professionals available at all times. Educating nurses to detect the early signs of bacterial infection and ensure they have the communication skills to relay the information to prescribers is an essential component for appropriately responding to suspected infection (Kullar et al., 2017; Fleet et al., 2014). Educational interventions targeting nursing staff and prescribers tend to include training for following guidelines for suspected infections, and sharing knowledge about the risk of inappropriate antibiotics and the spread of bacterial resistance. One of the described barriers to implementing ASPs in nursing homes is the lack of educational resources for antibiotic use and infection management (Dyar et al., 2015). Implementing educational interventions can ensure that nursing home staff follow IPAC policy and guidelines are adhered to effectively, such as hand hygiene awareness and use of IUCs (Koo et al., 2016). Thus, multidisciplinary education interventions can have a profound influence on inappropriate antibiotic prescriptions and can complement institutional IPAC programs (Koo et al., 2016; Dyar et al., 2015).

The necessity of multidisciplinary educational interventions remains a disputed matter. Although nurses play a significant role in prescribing antibiotics, high turnover rates may hinder the effectiveness of nurse-targeted educational interventions; thus, some may consider purely 
physician-targeted education interventions a better alternative (Mylotte, 1996). The efficacy of physician-only educational interventions has been displayed in a few studies but opposed by others. In two systematic reviews, education of prescribers was found to be an effective intervention for reducing CDI diarrhea rates (Marshall et al., 2017), improving antibiotic prescribing practices, and reducing consumption and costs (Corsonello et al., 2015). Other studies, however, did not find such results and have argued for the need of multidisciplinary educational interventions. Katz et al. (2017) reviewed educational interventions in nursing homes targeted at both nurses and physicians; such interventions led to better interdisciplinary communication, fewer urine culture send-offs for asymptomatic bacteriuria, and overall lower rates of antibiotic prescriptions. Morrill et al. (2016) systematically reviewed multiple ASPs in nursing homes. Although the quality of evidence for many reviewed studies was found to be weak, educational interventions that were exclusive to physicians only were not effective for reducing antibiotic use. Further studies must clarify the need for multidisciplinary inclusion for educational interventions.

The effectiveness of educational interventions is largely lacking in evaluation. Moreover, evidence supporting exclusively educational interventions is limited (Rawson et al., 2017). Many studies that have reviewed the effectiveness of educational interventions utilized multifaceted strategies (Nicolle, 2014; Morrill et al., 2016). A systematic review of ASPs in ambulatory care compared printed educational materials with other intervention designs (Arnold AR \& Straus SE, 2005). Three studies were reviewed that utilized educational materials without including a physician-related process, such as audit and feedback (Angunawela et al., 1991; Avorn \& Soumerai, 1983; Schaffner et al., 1983). None of the studies managed to significantly improve antibiotic prescriptions. This is in line with the aforementioned complexity of behavioural modification techniques (Michie et al., 2011). Alone, educational interventions can be effective for initiating ASPs; however, they are unlikely to provide lasting behavioural changes (Katz et al., 2017).

Although the effectiveness of certain, multifaceted educational interventions has been established, their sustainability is questionable. Nicolle (2014) reviewed ASPs in nursing homes, which included two RCTs. Significantly improved antibiotic use was demonstrated following educational interventions; however, significant improvement was not sustained after 15 months. Fleming et al. (2013) systematically reviewed RCTs for implementing educational interventions 
in nursing homes. The educational interventions incorporated prescribing feedback for physicians only. Though the odds of prescribing antibiotics nonadherent to guidelines was significantly reduced (odds ratio (OR) 0.47 and 0.48 ), the results were not sustained 6 months following the intervention. Likewise, Loeb et al. (2005) found similar sustainability results in a nursing home educational intervention targeted at both nurses and physicians. The intervention consisted of small group interactive educational sessions, one-on-one meetings with physicians, and treatment algorithms for UTIs. Significantly reduced rates of antibiotic prescriptions for UTIs were exhibited for the treatment group, yet the results were not sustained at 12-month follow-up. Improving intervention sustainability may require repeated interventions provided in a structure manner (Fleming et al., 2013; Katz et al., 2017). Therefore, although further research for educational interventions is necessary, an effective intervention in nursing homes ought to be structured and organized, possibly with incorporation of prescriber feedback, with consideration for sustainability (Nicolle, 2014; Loeb et al., 2005; Fleming et al., 2013; Katz et al., 2017).

\subsubsection{Formulary Interventions and Antibiotic Cycling}

Some ASP interventions have been designed to target the organization and use of formulary (i.e. lists of medications). Formulary review, a PHO Core Strategy, is an intervention that involves assessing the availability of antibiotics within an institution (Dellit et al., 2007). Typically, a pharmacy committee evaluates antibiotics for several criteria: effectiveness, safety concern, risk of spreading resistance, expense, and pharmacokinetics. ASPs can be divided into specialized interventions that target formulary and prescriptions of antibiotics: formulary automatic substitution and formulary restriction, both of which are PHO Core strategies, and antibiotic cycling (PHO, 2016).

In Ontario, all adults aged 65 and older receive drug benefits under the Ontario Drug Benefit (ODB) program. The formulary for such medication is included in the ODB formulary. Older adults residing in other jurisdictions may have their own drug benefit program and would be included in another antibiotic formulary. Thus, the differences in drug funding between nursing home residents of different jurisdictions may complicate the implementation of formulary interventions (The Institute for Clinical Evaluative Sciences (ICES), 2011).

Formulary automatic substitution is an intervention that allows pharmacists to automatically substitute an order of certain antibiotics (or any medication), in accordance with a preapproved 
procedure, without requiring prescriber consultation. A common application for formulary automatic substitution is the automatic replacement of nonformulary (restricted) antibiotics with those that are formulary and therapeutically similar. Certain antibiotics may be considered nonformulary due to their relationship with ADEs, expense, and the spread of resistance (PHO, 2016; Haas \& Bonczar, 1996).

Formulary restriction is a formulary ASP that utilizes the restriction of certain antibiotics and provides guidance for their use. Specific antibiotics ought to be restricted for several reasons: risk of developing resistance, risk of overuse/misuse, needed for treatment of MDRO infections, broad-spectrum, cost, and risk of ADEs (PHO, 2016). A similar intervention to formulary automatic substitution for replacing antibiotics is the use of antibiotic cycling. Antibiotic cycling involves repeated exchanging of antibiotics of a certain class to those of another class after a fixed interval, or cycle, as opposed to substituting antibiotics with those of the same class. Determining the duration of each time interval can be dependent on local susceptibility patterns or a predetermined length of time. The theory supporting antibiotic cycling is that overused antibiotics are more likely to develop resistance; thus, cycling between classes will reduce the selective pressures established during their use, and repeated for the next class. Cycling between classes of antibiotics may be effective for reducing resistance build-up; however, abandoning the use of particular antibiotics altogether may be an even more effective strategy (Bergstrom et al., 2004; Brown \& Nathwani, 2005) Overall, the evidence supporting antibiotic cycling is too weak to be recommended in acute hospital and nursing home care (Brown \& Nathwani, 2005).

Formulary interventions can be implemented individually or bundled together as a multifaceted intervention. Formulary interventions may aid in the prevention of ADEs, expense of treatment, and the spread of resistance; however, evidence supporting their effectiveness is mixed (Haas \& Bonczar, 1996; PHO, 2016). Evidence describing the implementation of formulary review and automatic substitution interventions is limited. In Arnold \& Straus' (2005) systematic review of ASPs in ambulatory care, financial and health care system changes were compared to other interventions. A component of such system changes included updating the provincial drug formulary to restrict fluoroquinolone use for older adult patients. A significant drop in average monthly fluoroquinolone use was reported after implementation of the intervention. A systematic review of ASPs implementing antibiotic cycling interventions in hospitals identified four studies appropriate for review. Although positive results were discovered, flaws in the methodology and 
standardization inhibit conclusions from being drawn (Brown \& Nathwani, 2005). In Marshall et al.'s (2017) systematic review of ASPs in hospitals for CDI, formulary restriction interventions that restricted broad-spectrum antibiotics for all infections except CDI had a significant effect on reducing incidence of resistant bacteria. In another systematic review of ASPs in EDs, formulary restriction was successful for significantly reducing antibiotic use (Losier et al., 2017). Although formulary interventions have been supported in hospital settings, they have been inadequately explored in nursing homes and further studies are necessary prior to concluding their widespread effectiveness and feasibility (Schuts et al., 2016).

\subsubsection{Guidelines for Empiric Prescribing}

Empiric prescribing of antibiotics is almost always the practice in nursing homes; in many instances, empiric prescribing of antibiotics is required in nursing homes due to the unavailability of diagnostic testing (Medina-Walpole \& McCormick, 1998). The lack of on-site laboratories, on-site consultation, and other diagnostic testing in nursing homes often results in difficulty identifying the infecting organism; thus, resulting in the need of empiric antibiotic therapy (Dyar et al., 2015; Katz et al., 1990). Empiric selection of initial antibiotic therapy by prescribers can be a challenging process; hence, the need for interventions that implement guidelines for empiric antibiotic therapy. Empiric antibiotic prescribing guidelines are beneficial to prescribers for providing a standardized method for selection of initial antibiotics, such as choosing which broad-spectrum antibiotics ought to be used, and guidelines for transitioning to other antibiotics when necessary (Schwartz et al., 2007; PHO, 2016). ASP interventions introducing empiric antibiotic prescribing guidelines in hospitals have shown promising results, such as improved clinical outcomes, reduced adverse events and costs, but results have been heterogeneous and inconsistent. While somewhat studied in hospitals, relevant studies of empiric antibiotic prescribing guideline interventions have not been identified and reviewed sufficiently for nursing homes (Schuts et al., 2016).

\subsubsection{Intravenous to Oral Antibiotic Conversion}

Antibiotics are generally administered either intravenously (IV) or orally. Most antibiotics that are given via IV already have high bioavailability, a pharmacokinetic property that refers to the quantity of the unchanged drug that reaches the targeted area (Griffin, 2009). Antibiotics with high bioavailability do not need to be administered via IV because they are capable of treating 
the targeted region through oral ingestion (PHO, 2016). Oral antibiotics are far less expensive than IV; a study of 128 Veterans Affairs hospitals found that potential cost savings for transitioning IV antibiotics to oral antibiotics over a four-year period approximated $\$ 4$ million. Transitioning IV to oral antibiotics provides numerous benefits to patients such as reduced catheter-related infection, hospitalization, and workload, without negatively impacting health outcomes (Goff et al., 2012). Additionally, many antibiotics that are provided appropriately via IV can eventually be transitioned to oral intake when the patient's clinical condition is improved (PHO, 2016). Thus, ASP interventions can target the transition of unnecessary IV antibiotics to those provided orally when clinically indicated. Guidelines can be implemented into policy providing direction for when to transition antibiotics; this can be in alignment with guidelines for selection of antibiotics, determining duration of treatment, and other stewardship initiatives (Naughton et al., 2000). Transitioning from IV to oral antibiotics is included as a PHO Core Strategy; such an intervention has numerous benefits for both patients and overall expense of treatment (PHO, 2016). However, evidence supporting such an intervention is not conclusive. In Schuts et al.'s (2016) systematic review of ASPs, conversion of antibiotics from IV to oral was associated with improved clinical outcomes, reduced ADEs, and cost of therapy, but the findings were insignificant. Additionally, few reviews of interventions for transitioning IV antibiotics to oral in nursing homes were found. Thus, further studies, particularly in nursing homes, are required to determine the value of such an intervention.

\subsubsection{Multifaceted Interventions}

Behaviour modification is a complex task and, thus, requires complex interventions (Craig P, 2008). Multifaceted ASP interventions utilize the bundling of two or more strategies to form a complex intervention (Morrill et al., 2016). Multifaceted interventions often apply education as their foundation and incorporate other strategies. Strategies incorporated may include reminders, guidelines, decision aid cards, antibiograms, checklists, academic detailing, and audit and feedback. Multifaceted interventions can be multidisciplinary and inclusive to physicians, nurses, HCPs, and patients and their advocates (Arnold \& Straus, 2005; Fleming et al., 2013; Loeb et al., 2005). Results from previous studies have demonstrated the necessity of multidisciplinary inclusion, including multiple levels of staffing in the intervention. Implementation of physicianonly interventions have not been reliably effective for reducing overall antibiotic use (Monette J, 2007; Schwartz et al., 2007). Considering the substantial role nurses and other front line health 
professionals play in antibiotic prescriptions, it is intuitive that they be included in ASPs, particularly for interventions as complex as multifaceted interventions (Morrill et al., 2016). Such an approach has been supported by several systematic reviews of ASPs; however, the support for multifaceted interventions is not universal (Feldstein D et al., 2017; Naughton et al., 2001).

The practicality of multifaceted interventions is not clear; many studies have described such interventions to be significantly superior to singular interventions but others have not drawn the same conclusions (Arnold \& Straus, 2005; Tonkin-Crine et al., 2017). Zabarsky et al. (2008) implemented a multifaceted educational intervention to reduce prescriptions of antibiotics for asymptomatic UTIs in nursing homes. The multifaceted intervention incorporated direct feedback for nurses and physicians and saw a significant decrease in prescriptions maintained for 30 months following the intervention. Another systematic review of ASPs in nursing homes examined the value of multifaceted interventions (Nicolle, 2014). The multifaceted interventions included incorporating small group educational sessions, audit and feedback of infection management practices, local hospital resistance, guidelines, and written materials. One of the two multifaceted interventions utilized small group educational sessions and found improvements in average antimicrobial starts and monthly antimicrobial days that were sustained for 2 years. However, the second multifaceted intervention, which utilized guidelines, written materials, and prescriber feedback, did not find significant improvements for fluoroquinolone use. These contrasting findings are likely due to intervention design differences; certain interventions incorporated into multifaceted interventions may be more effective than others. However, identifying the individual successful components within a multifaceted intervention is a troublesome task, as studies are scarce and teasing out the effective components and their relative contribution is challenging (Zabarsky et al., 2008; Arnold \& Straus, 2005; Tonkin-Crine et al., 2017; Nicolle, 2014).

A primary concern regarding implementing multifaceted interventions is feasibility. Implementing complex interventions, such as multifaceted interventions, requires significant resources, which may not be available in nursing homes and may hinder their sustainability. Studies investigating the feasibility of multifaceted interventions are lacking and will be required to conclude their practicality (Bond et al., 2014; Stirman et al., 2012; Katz et al., 2017). 


\subsubsection{Short Course Antibiotic Therapy}

Antibiotics that are prescribed for longer durations or that consist of multiple course are linked to higher incidences of antibiotic resistance (Costello 2010). ASP interventions can be implemented to reduce unnecessary antibiotics and introduce short course antibiotic therapy. Long antibiotic durations are often the most unnecessary antibiotic prescriptions and are the major cause of overconsumption in acute care settings, which may be the case in nursing homes as well (Hecker et al., 2003; Peron et al., 2013). It has been found that $2 / 3$ antibiotic durations in nursing homes exceed 10 days. Most antibiotic therapy durations generally fall between 5 and 10 days. Short course antibiotic therapy interventions can decrease treatment duration to 3 days (Lutters \& Vogt-Ferrier, 2008). Although optimal duration of antibiotic therapy is disputed (Gray \& Malone-Lee, 1995), the IDSA considers short course antibiotic therapy a highly recommended ASP intervention with moderate quality of evidence (Barlam et al., 2016; Lutters \& Vogt-Ferrier, 2008; Vogel et al., 2004).

Lutters \& Vogt-Ferrier (2008) systematically reviewed ASPs implementing short course antibiotic therapy for treatment of uncomplicated symptomatic UTIs in older adult women. Although the quality of evidence was questionable (aside for a study by Vogel et al. (2004)), short course antibiotic therapy was found to be as effective as standard and long course durations for treatment of uncomplicated symptomatic UTIs. Vogel et al. (2004) conducted a double-blind RCT testing the effectiveness of short course antibiotic therapy in acute care and community settings. Only older adult women diagnosed with uncomplicated symptomatic UTIs were included. The intervention group received ciprofloxacin $(250 \mathrm{mg})$ orally twice a day for three days, which were replaced with placebos for the remaining four days. The control group received the same prescription of ciprofloxacin for seven days. Short course antibiotic therapy was found to be as effective for eradicating bacterial infection at two-day follow-up as standard course therapy and significantly more effective for reducing ADEs. Daneman et al. (2013) examined the causes of prolonged antibiotic durations in nursing homes. Prescriber characteristics were found to be more predictive of prolonged durations than resident characteristics. Specifically, prescribers with past tendencies to prescribe lengthy durations of antibiotics were more likely to prescribe lengthy future durations of antibiotics. Thus, short course therapy interventions could be implemented to target prescribers with tendencies to prescribe prolonged antibiotic therapy durations (Daneman et al., 2017). Short course antibiotic therapy may be as effective for 
treatment of infection as standard and long course antibiotic therapy; however, research investigating short course antibiotic therapy interventions, particularly in nursing homes, is limited and should be addressed in the future (Barlam et al., 2016).

\subsubsection{Targeting CDI}

Targeted review of patients with CDI and reducing antibiotics associated with MDROs is an important ASP intervention for limiting the spread of bacterial resistance. Approximately 619,000 cases of CDI can be prevented in the US over a 5-year period with immediate implementation of ASP interventions (Slayton et al., 2015). Certain antibiotics are considered high-risk for the spread and acquirement of CDI, such as clindamycin and broad-spectrum antibiotics including cephalosporins and fluoroquinolones. Restricted access to such antibiotics is a strategy for reducing the risk of spreading and contracting CDI. Other efforts can be centered on improving appropriate antibiotic therapy through adherence to guidelines, educating health care staff about the risks of CDI, providing treatment recommendations, and reviewing management of infection (Barlam et al., 2016). On-site nursing home ID consult service can be implemented to improve utilization of antibiotics; the team can visit patients prior to suggesting prescribing recommendations, such as antibiotic dosing adjustment and tailoring antibiotics to culture results (Jump et al., 2012). Reducing rates of CDI can be accomplished through many strategies; nevertheless, interventions for targeting CDI and MDROs have their challenges (Marshall et al., 2017; Patton et al., 2017).

Interventions for targeting CDI and MDROs are recommended by the IDSA and exhibited moderate quality of evidence (Barlam et al., 2016). Interventions targeted at CDI have been found to be effective for reducing rates of CDI, high-risk antimicrobials, time-delays for treatment of confirmed cases of CDI, and improved adherence to treatment guidelines (Patton et al, 2017; Jury et al., 2013; Welch et al., 2016; Jump et al., 2012). Jury et al. (2013) implemented an ASP targeting CDI at a hospital and adjacent nursing home in Cleveland, OH. The intentions of the intervention were to reduce time-delays in initiation of antibiotic therapy for patients with CDI, improve adherence to treatment guidelines by the IDSA (Cohen et al., 2010), and reduce empiric therapy for suspected but undiagnosed CDI. The intervention included five components: (1) education of physicians for diagnosing and treating CDI; (2) a CDI order menu; (3) communication between microbiology lab and ASP team for all positive CDI results; (4) review of EMR by the ASP team, recommendations for initiation of therapy, and feedback for 
noncompliant prescribers; and (5) review of EMRs for patients prescribed antibiotics for CDI empirically. Findings of the study included significantly reduced time-delays for initiation of antibiotic therapy and significantly improved adherence to treatment guidelines. Not all reviews of ASPs for reducing CDIs have had significant results. In Marshall's (2017) systematic review of interventions for reducing CDI, few interventions significantly reduced CDI diarrhea rates: formulary restriction of broad-spectrum antibiotics for infections other than CDI, implementing IPACs, audit and feedback programs, and prescriber education. Interventions that were not effective included antibiotic policy changes, procedural changes, and use of probiotics. Thus, interventions intended to reduce the spread of CDI must consider which interventions are found to be successful; however, further reviews are needed to confirm such findings.

\subsection{Rationale}

All health care facilities are recommended to implement ASPs; however, implementing an ASP intervention is not a simple process. Facilities need access to available literature and guidelines to review potential interventions and select those that are feasible to implement and likely to produce reliable positive outcomes. ASP implementation in acute hospital care has been well documented with many systematic reviews and meta-analyses, although no randomized controlled trials have demonstrated benefit (Schuts et al., 2016; Wagner et al., 2014). Such an evidence-base has benefitted hospitals in the selection and implementation of tested and supported interventions and has resulted in improved antibiotic utilization. Nursing homes are primary candidates for ASP implementation. Nursing home residents are highly vulnerable to infection and inappropriate antibiotic prescriptions; yet, ASP research in nursing homes remains limited. Individual ASP designs have not been studied nor contrasted well enough to determine conclusively which interventions are more likely of resulting in lasting change in nursing homes. Thus, nursing homes are in need of another method for selecting and implementing ASPs that are feasible for their facility. The Modified Delphi Method may be the solution for aiding nursing homes in their selection and implementation of feasible ASPs. 


\section{Conclusions}

Antibiotics are often inappropriately prescribed in nursing homes. Prescriptions of antibiotics are frequently made without indication of infection. Physicians in nursing homes often prescribe antibiotics empirically, without microbiological support for identification of the infecting organism. Inappropriate antibiotic consumption is an important cause of adverse drug events and is a chief driver for the spread of antibiotic resistance. Thus, improving antibiotic use through bettering prescribing practices is essential for reducing the risk of ADEs (i.e. injuries as a result of medication use) and halting the spread of bacterial resistance.

Antimicrobial stewardship programs refer to efforts and interventions implemented in health care settings to optimize the use of inappropriate antimicrobial and antibiotic use, and reduce ADEs and health care expense. ASPs have been implemented into many divisions of health care. Nursing homes are a prime candidate for ASP interventions, as antibiotic use is prevalent and often inappropriate. Additionally, nursing home administrators have displayed interest in the implementation of such interventions; yet, ASP implementation in nursing homes remains mostly underexplored relative to other health care institutions, such as acute hospital and ambulatory care.

Health care settings such as acute hospital care have access to resources such as diagnostic testing tools, IPAC programs, and data tracking. Such access to resources is limited in many nursing homes. Furthermore, individual nursing homes differ in their access to such resources; thus, generalizing ASPs for all nursing homes is a challenging venture. ASP research in nursing home settings is limited. Systematic reviews of ASPs are far more abundant in acute care and emergency department settings than nursing homes. ASPs have been highly recommended in nursing home settings by organizations such as the IDSA, CDC, SHEA, and PHO; however, guidelines for their implementation are lacking. Thus, evidence supporting the selection and implementation of appropriate ASP interventions in nursing homes is critical.

Implementing ASP interventions into health care is often a tedious and resource-intensive task. ASP interventions have been developed to address the many specific components of the prescribing process, such as educating patients and their advocates, and utilizing behaviour modification techniques to improve physician antibiotic prescribing rates. Interventions can be

implemented individually or bundled in a multifaceted intervention. Several interventions were 
presented previously, which were identified from systematic reviews and organizational publications (PHO, IDSA, CDC, etc.): antibiograms, antibiotic cycling, audit and feedback, clinical decision support systems, de-escalation, education, formulary automatic substitution, formulary restriction, formulary review, guidelines for empiric prescribing, IV to oral transition, multifaceted interventions, short course antibiotic therapy, and targeting CDI.

The evidence supporting each intervention individually and multifaceted is unclear and inconsistent. An intervention may be found to be effective in one systematic review for reducing unnecessary antibiotic use and unsuccessful in another. Communicating the supporting evidence of interventions may not be an effective method for implementing ASP interventions in nursing homes. Additionally, extrapolating evidence from other health care settings, such as acute hospital care, to nursing homes has its limitations. Differences such a length of stay, staff turnover, and access to resources hinder the generalizability of ASP research in hospital care to nursing homes. Nursing home residents would benefit from ASP implementation; however, not enough work has been done to evaluate ASPs in such settings. The evidence supporting individual interventions over others in nursing homes is inconclusive. ASP interventions need to be evaluated and prioritized to assist nursing homes in their selection and implementation. The Modified Delphi Method may be the solution to providing nursing homes with such resources (Kullar et al., 2017; Matheï et al., 2007; Sloane et al., 2016; Malani et al., 2016; Dellit et al., 2007; Morris et al., 2012; Fitch et al., 2001). 


\section{Study Purpose, Objectives, and Intended Outcomes}

\subsection{Purpose}

Nursing home providers lack guidance in the selection and implementation of ASP interventions, and this is not supported by existing research. Implementing ASP interventions in nursing homes can be heavily resource-intensive; however, nursing homes are generally lacking in access to resources. Effort is needed to evaluate ASPs for nursing homes and describe the resources needed for implementation in such settings. The purpose of this thesis is to utilize the Modified Delphi Method to evaluate and prioritize ASP interventions for nursing homes and describe the necessary resources for their implementation.

\subsection{Objectives}

An interdisciplinary, national expert and non-expert panel was invited to participate in the Modified Delphi panel. The panel completed two rounds of surveys and discussed their thoughts regarding individual and multifaceted nursing home ASP interventions. The interventions were evaluated across several criteria including scientific merit, impact, feasibility, accountability, and importance. The interventions were grouped into two categories: (1) not necessary or essential for nursing homes, and (2) necessary and essential for nursing homes. The interventions in the second group were prioritized for nursing home settings and the resources required for implementing each were described.

\subsection{Intended Outcomes}

The intended outcome was to provide nursing homes with two tools for implementing ASP interventions:

(1) A prioritized list of interventions considered necessary and essential for nursing homes to aid in their selection and implementation of ASP interventions and,

(2) The resources required to implement each intervention 


\section{Chapter 2 \\ Materials and Methods}

\section{Key Objectives}

(1) To provide an overview of the Modified Delphi Method, including participant identification and invitation,

(2) To describe documents prepared for panel members and survey construction, and

(3) To describe planning for the in-person Delphi meeting, including reviewing survey results, resource discussion, prioritization, and End of Day Survey. 


\section{The Modified Delphi Method}

The Delphi Method is a structured, interactive consensus tool that applies expert opinion in a panel setting. The method was designed by the RAND (Research and Development) Corporation, initially for military and political purposes but since has been used for medical research as well. An overview of the Modified Delphi method (or RAND/UCLA Appropriateness Method (RAM)) is illustrated in Figure 3 (Fitch et al., 2001). The process generally includes anonymous questionnaires sent in rounds. Responses are collected and participants are sent the questionnaire again with the opportunity to change their answers. The purpose of the multiple rounds is to iteratively narrow down the answers until arriving at consensus.

The Modified Delphi Method differs from the original Delphi method in its inclusion of expert discussion. Generally, a Modified Delphi process begins with respondents completing the first round of questionnaires on their own prior to the assembly of an in-person panel meeting. During the day of the in-person panel meeting, the panelists receive a document summarizing the results from the first round. Panelists are then encouraged to discuss their perspectives and areas of disagreement with the results. Upon completion of the discussion, the second round of questionnaires is given to the panelists and the scores for each item are calculated. Depending on their score, items are then classified as "appropriate", "uncertain", or "inappropriate". Although Modified Delphi panels have generally been used for the purpose of selecting indicators for performance, particularly for medical procedures, they are not limited to indicators and can be used for medical interventions, specifically ASP interventions (Dalkey \& Helmer, 1963; Fitch et al., 2001; Moehring et al., 2017).

The Modified Delphi Method has its strengths and limitations. As it incorporates in-person discussion, the Modified Delphi Method provides panel participants an opportunity to meet each other and express their perspectives and opinions in an open environment. However, with inclusion of face-to-face interaction in the Modified Delphi Method also raises the possibility of biases. Panel participants are more likely to be intimidated and agree with other panelist in person than they would be online. However, this bias can mostly be controlled with effective leadership; thus, arguing the need to select an experienced facilitator (Kahan et al., 1994). 
One of the chief limitations of the Delphi Method is the general lack of a theoretical framework. Though Delphi panels often have overlapping methodology, the technique is not constant in its application. Ambiguities are common when organizing a Delphi panel, such as panel size, gathering expert opinion, and determining consensus. Guidelines for recruiting the expert panel are unclear. The size and composition of the panel vary between studies and areas of interest. The methodology for gathering expert opinions, such as choice of Likert scale (i.e. using 5-point vs. 9-point), is inconsistent. Lastly, means of determining consensus varies and depends on the specific study and its feasibility. Determining the specific number of Delphi rounds can be difficult. The choice of Delphi rounds varies widely in the literature, some from as little as two or three, up to ten. Thus, designing a Delphi panel can be complicated, especially without an underlying theoretical framework (Habibi et al., 2014). For the purpose of this thesis, past similar studies that utilized the Modified Delphi Method will be used as a theoretical framework (Morris et al., 2012; Dresser et al., 2017).

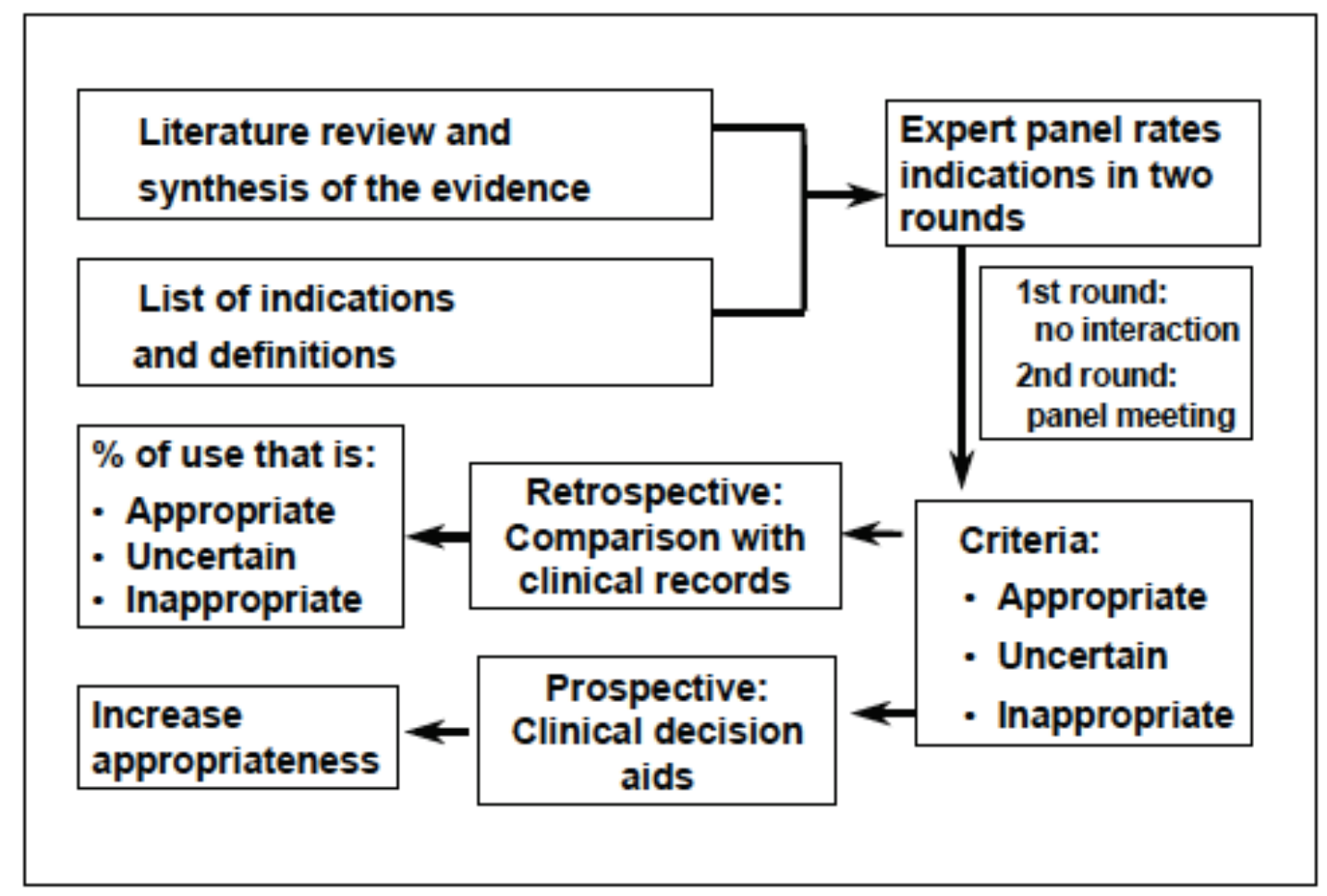

Figure 3. The RAND/UCLA Appropriateness Method (RAM)

Reproduced with permission from the RAND Corporation 


\subsection{Process Overview}

We utilized the Modified Delphi Method based on the RAND/UCLA Appropriateness Method to evaluate ASP interventions for nursing homes. An expert panel was identified and recruited for a one-day consensus meeting. We conducted a literature overview and developed a background package to provide panel participants with a review of the literature relating to ASP research in nursing homes. Two weeks prior to the date of the panel, panelists were provided the background package and the first round of a two-part anonymous survey. The survey required evaluation of ASP interventions in nursing homes. Results of the evaluation were collected and summarized for the in-person Delphi meeting. On the day of the in-person Delphi meeting, the survey summary was reviewed and interventions obtaining consensus for disagreement were eliminated following a brief discussion. The remaining interventions were reviewed and discussed individually. Following discussion, panelists were invited to complete the second round of the survey and were encouraged to alter their initial responses based on the discussion. Consensus was welcomed but not enforced. Results from the second survey round were tabulated and presented to the panelists. Several interventions were eliminated from discussion depending on their score from the second survey round. The remaining interventions were discussed for resourcing requirements and were ranked for priority. Upon conclusion of the meeting, a survey was provided to the panelists to ensure opinions were given honestly, the panel discussion was fair, and intimidation was not the cause of the revised answers.

\subsection{Identification of Candidate Panel Members}

The assembly of panel participants was critical toward achieving a successful Modified Delphi process. The ideal number of participants was unclear. Originally, Modified Delphi panels consisted of 9 panelists; this number was chosen because it was large enough for professional representativeness but small enough for the group to engage in discussion freely. However, recruiting exactly 9 participants can be challenging and is not necessary (Fitch et al., 2001). Considering the variety of staffing involved with ASP implementation in nursing homes, we aimed to recruit 12-15 panel members with national representation and role diversity (Dellit et al., 2007; Grol \& Grimshaw, 2003; Sloane et al., 2016). 


\subsubsection{Professional Representativeness}

To ensure consensus discussions and conclusions were valid, it was critical that the panel be multidisciplinary and have wide professional representativeness. We constructed a 'table of representativeness', which included variable professional roles desired for inclusion in the panel: ID specialists, nursing home physicians, infection control (IC) specialists, hospitalist MDs, other MDs, nurses, pharmacists, administrative staff, policy and stakeholders, and ASP experts. Beyond expert representation, another important representation we sought to include in the panel was nursing home residents, their families and/or advocates, and family councils. A growing technique for improving health care services is the use of patient and user input. This approach utilizes patient, advocate, and caregiver input to improve quality of care. In the past, patients and their advocates would play a more passive role as recipients of health care services. With this technique, improvements can be made that utilize greater perspective, without the concern of losing standardized, high quality methodology (Boyd et al., 2012). As previously discussed, patient and family pressure is a large contributing factor to inappropriate antibiotic prescriptions in nursing homes. Thus, including residents, families, and family councils in our panel provided greater perspective for evaluating ASPs in nursing homes.

Several members from the research team were involved in the panel participant identification process. The intention of the study was to utilize a professionally and geographically diverse expert and non-expert panel to evaluate ASP interventions. Thus, identifying individuals of a variety of professional roles from across the country was our aim. ASP experts and nursing home health care professionals were identified for invitation using previous ASP Delphi studies (Morris et al., 2012; Dresser et al., 2017). Additionally, various health care organizations were contacted for nominated participants, particularly for nursing home physicians, nurses, and residents: Family Councils Ontario (FCO), Ontario Long Term Care Association / Clinicians / Physicians (OLTCA / OLTCC / OLTCP), and Ontario Association of Residents' Council (OARC). Further ASP research experts were identified from the literature; some candidate participants were nominated via word of mouth. A list was compiled of potential panel members, which was provided to other members of the research team for review. 


\subsubsection{National Representativeness}

Outcomes from this Modified Delphi panel were intended to be applicable nationally; thus, recruitment of panelists from outside Ontario was desirable. To identify potential panelists, we contacted national organizations, such as the Canadian Association for Long Term Care (CAHLTC). For ASP experts outside of Ontario, the literature was reviewed and out-of-province experts were identified and invited to participate.

\subsection{Invitations to Candidate Panel Members}

\subsubsection{Setting a Time and Place}

Prior to sending out invitations, a date for the panel meeting was agreed upon that fit the research team's schedule, which was initially February 13, 2018 but was postponed to March 20, 2018. Modified Delphi meetings typically last a full day (Fitch et al., 2001). A room was reserved at the Sinai Health System that was large enough to comfortably fit the team and the panel members (assumed to be 20 attendees in total).

\subsubsection{Invitations}

An initial list of candidate panel members was organized and sent for review to members of our research team uninvolved with recruiting panel members. The initial list was evaluated; individuals with appropriate professional designations and relevance to nursing homes were included in the final list of invitations. Initial contact with candidate panel members was made via email. The email consisted of a brief description of the intentions of the Modified Delphi Method and an official invitation was appended to the email. The official invitation consisted of three components: (1) a brief introduction for the need of ASP interventions in nursing homes, (2) the intentions of the Modified Delphi process to evaluate and contrast ASP interventions in nursing homes, and (3) the role of the panel members for achieving the goals (completion of surveys and in-person discussion). Details for the time and location of the in-person meeting were provided in the official invitation. Motivation to participate in the Modified Delphi panel included acknowledgements in any reports and publications. Travel expenses were to be reimbursed. Participants were requested to provide their response no later than one week after receiving the invitation. 


\section{Ethics}

Research Ethics Board approval for this Modified Delphi panel was obtained from the Sinai Health System on March 5, 2018. Individuals agreeing to participate in the panel were provided a consent form to be signed and delivered to the researcher prior to the day of the meeting (March 20, 2018). There were several components to the consent form: an introduction to the study, a background summary of the literature and purpose of the Modified Delphi panel, the study design, study visits and procedures, risks and benefits related to participating, the voluntary nature of the study, confidentiality, actions in case of harm, expenses, and questions about the study.

Prior to receiving and signing the consent form, panelists were described (via email) the nature of the study and their option to decline participating in the panel without any negative consequences. Panelists were sent the consent form over email to read in full and ask any questions. The consent form was read, signed, and dated by the panelists, then delivered to the researcher to sign and date, and then a copy of the form was given to the panelists.

Panelists were invited to complete two rounds of surveys. An ethical consideration was to ensure confidentiality of data and survey responses. SimpleSurvey ${ }^{\mathrm{TM}}$, a Canadian-based and hosted survey development software, was used to create the surveys (https://simplesurvey.com). Survey responses were collected anonymously. Three panelists did not have access to a computer and completed the survey on paper. To provide a level of confidentiality, no identifiers were included in the paper surveys and the completed surveys were delivered to the analysts randomly. Any information collected for the study is kept in a locked and secure area by the researcher for 10 years.

Panelists were not placed in any form of harm by participating in the Modified Delphi panel. A time commitment for completion of the surveys and attendance at the in-person meeting was required. Potential benefits for participation included information discovered through the Modified Delphi panel process and acknowledgements in future papers. 


\section{Background Package}

Two weeks prior to the date of the Modified Delphi panel, panelists were sent a background package. The background package consisted of a literature review, the first round of a two-round survey, and a conflict of interest form.

\subsection{Introduction and Brief Literature Summary}

An introduction and brief literature summary was provided to panelists. The literature described nursing homes, infections in nursing homes, antibiotics in nursing homes and antibiotic inappropriateness, bacterial resistance, and the need for ASP interventions. Interventions were identified via government agencies, such as IDSA and PHO, and a literature overview. Interventions were tabulated, including the name of the specific intervention and its description (Table 1). Although the scientific evidence supporting each intervention varied, both for particular health care settings (nursing home vs. hospital) and overall effectiveness, we chose to be as inclusive as possible and include all identified and relevant ASP interventions in the package for evaluation and discussion.

The three intentions of the Modified Delphi panel were explained: (1) to provide a list of relevant ASP interventions for nursing homes, (2) identify resource requirements for such interventions, and (3) prioritize the ASP interventions that are most necessary and essential for nursing homes. Systematic reviews were collected for evaluation of ASP interventions in and out of nursing homes (hospitals, EDs, ambulatory care). The systematic reviews were summarized and key findings were highlighted (Appendix A) (Nicolle, 2014; Katz et al., 2017; Fleming et al., 2013; Tonkin-Crine et al., 2017; Losier et al., 2017; Baur et al., 2017).

\subsection{Survey - Round 1}

For development of the survey, we used an online data collection and analysis tool (SimpleSurvey ${ }^{\mathrm{TM}}$ ). A brief review of the study objectives and a guideline for completion of the survey were provided. In the first section of the survey, panelists were asked to review and score five criteria for evaluating the interventions: (1) scientific merit, (2) impact, (3) feasibility, (4) accountability, and (5) overall importance of the intervention. Such evaluative criteria have been successfully used in previous similar studies (Morris et al., 2012; Dresser et al., 2017). Panelists 
were invited to provide commentary for the evaluative criteria. A 9-point Likert-Type scale was utilized; a rating of 1 indicated strong disagreement with the evaluative criteria and 9 indicated strong agreement. ASP interventions were presented individually, including a short description, followed by evaluation with the five criteria. Panelists were given an opportunity to provide comments for each intervention. Upon completion of the survey, panelists were requested to provide last additional comments, particularly, any potentially missing interventions. Panelists were requested to complete the survey no later than one week after delivery of the background package. Responses were collected anonymously (Appendix B).

\subsection{Conflict of Interest Form}

To reduce the risk of bias, panelists were requested to complete a potential conflicts of interest form. Panelists were asked to comment on their financial and other relationships with bodies that could be perceived to influence their contributions toward the Modified Delphi panel for assessing ASP interventions in nursing homes. The source of our funding was described. The completed forms were returned via email or provided in-person at the panel meeting (Appendix C).

\subsection{In Preparation of the Panel Meeting}

After the first round of the survey was completed, interventions were scored for the evaluative criteria and responses were retrieved from SimpleSurvey ${ }^{\mathrm{TM}}$. Scores were analyzed and divided into three categories: (1) scores ranging from 1-3 were considered disagreement, (2) scores ranging from 7-9 were considered agreement, and (3) scores ranging from 4-6 were considered uncertain. An agreement of $75 \%$ was determined to be a consensus. This method for analyzing the survey scores has been successfully used in previous Delphi panels (Morris et al., 2012). A summary document was constructed describing the results for each of the interventions for each criteria and were presented to the panelists on the day of the panel.

\section{Panel Day}

The panelists met for the Modified Delphi panel on March 20, 2018 at 9:00am. Panelists were provided with a folder containing several documents pertinent to the meeting: an agenda for the day's events, a summary table describing the results from the first round of the survey, the 
second round of the survey with each intervention on a different coloured paper, and an End of Day Survey. A brief overview of the agenda for the day was reviewed (Appendix D). The facilitator was Dr. Andrew Morris, the Medical Director of the Sinai Health System/University Health Network Antimicrobial Stewardship Program. The facilitator introduced himself and invited the panelists and researchers to introduce themselves, the organization they represent, their occupation, their reason for attending, and their expectations for the day. Following introductions, the facilitator presented a brief discussion of the background literature, explained the purpose for the Modified Delphi panel, and provided an overview of the process (Figure 4). The panel was then steered toward reviewing the results from the first round of the survey as a whole; interventions were then reviewed individually.

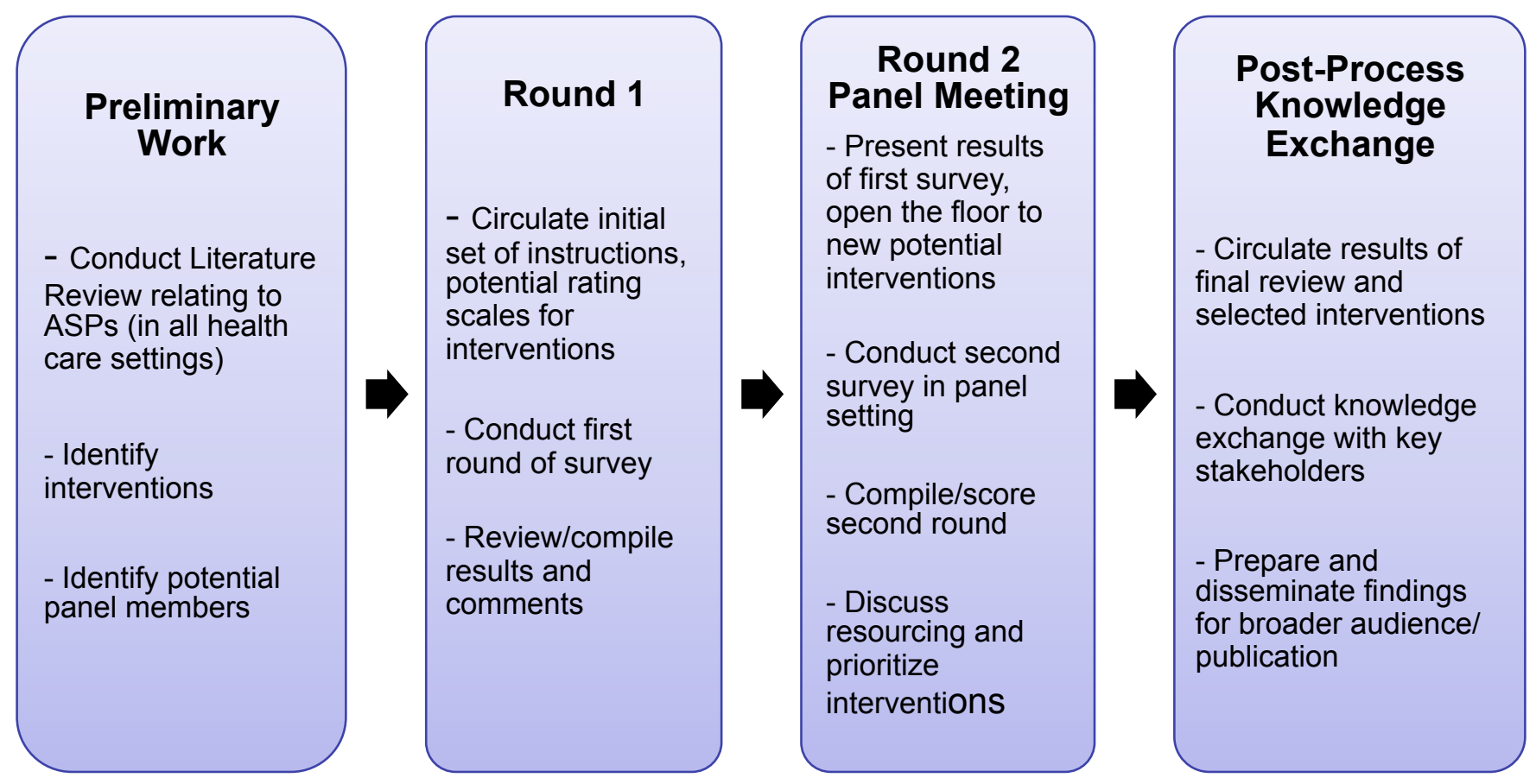

Figure 4. Overview of the Modified Delphi Process 


\subsection{Review of Survey 1 and Completion of Survey 2}

The evaluative criteria were briefly discussed prior to reviewing the first round of the survey. Comments for the evaluative criteria were addressed and further questions and comments were welcomed. The first component of the intervention review was to address interventions that received consensus for elimination (disagreement) and to have them removed from further discussion. The second component of the intervention review was to address interventions that received consensus for agreement or were of uncertain agreement. Interventions were addressed individually and their results across the five evaluative criteria were presented. Comments received from the first round of the survey were displayed. Following review of the results for the intervention of interest, the panel was open to discussion and debate. Points to ponder were provided by the facilitator with consideration to not induce a biased response. Discussion for the intervention was completed when the panel agreed that all points had been addressed and the panel could proceed to reevaluation (Survey 2). Panelists were guided to open their folders, remove and complete the coloured paper containing the evaluative criteria for the intervention that had just been discussed. Panelists were encouraged to revise their previous answers based on the discussion. Individual intervention surveys were completed anonymously and results were entered onto a spreadsheet. The cycle repeated for the rest of the interventions; individual interventions were presented with their results from the first survey round, open discussion was encouraged, surveys were completed, and results were entered onto the spreadsheet. Once the results were finalized, a slide deck was organized to display the scores for each intervention and compare between the first and second survey round.

\subsection{Post-survey Discussions}

\subsubsection{Final List of ASP Interventions for Nursing Homes}

The results from both rounds of the survey were displayed per intervention. The interventions were reviewed individually; interventions that obtained consensus for agreement were retained and those obtaining consensus for disagreement were eliminated. Interventions that did not obtain consensus for either agreement or disagreement were discussed and either reserved or eliminated. 


\subsubsection{Resourcing}

Upon completion of the final list of ASP interventions deemed appropriate and necessary for nursing homes, the panel discussion was then led to the topic of resourcing. Financial and professional resourcing was discussed for each intervention. Panelists were encouraged to consider technical resourcing, such as electronic support and best practice tools, and human resourcing for the final interventions.

\subsubsection{Prioritization}

Upon completion of discussing resourcing, panelists were encouraged to provide their thoughts for prioritizing the interventions. Panelists were reminded to consider the resource requirements for each intervention and reorganize the list of interventions from highest priority to lowest priority. Although evidence supporting the interventions ought to be considered, panelists were reminded that much evidence for ASPs in nursing homes is lacking altogether and should not be the sole determinant for prioritization. Large adhesive-backed posters were placed around the room for the remaining interventions. Panelists were asked to rank each intervention by placing a number (between 1 and the number of final interventions) on each poster.

\subsection{End of Day Survey}

Following completion of the discussion and prioritization of the ASP interventions, panelists were provided with an End of the Day Survey (Appendix E). The purpose of the survey was to understand how the panelists perceived the Modified Delphi panel process. The survey was divided into three parts: (1) the background package, (2) in-person meeting, and (3) additional comments.

Questions addressing the background package were designed to ensure the panelists understood what was expected during the in-person meeting. The panelists differed from each other in their experience with ASPs and nursing home care. Inclusion of background information was essential for ensuring that panelists shared a similar depth of knowledge to understand their opinions and share their thoughts during the in-person meeting. Instructions, expectations, and formatting were assessed for clarity; questions were evaluated for comprehension; and timing was gauged for adequate completion of the survey. 
Questions addressing the in-person meeting were divided into two sections: questions relating to the discussion, and questions regarding the second round of the survey. Questions relating to the discussion were designed to assess panelists' experience with expressing their thoughts and opinions. Panelists were asked to rate their experience with feeling respected, heard, and understood. Panelists were asked to rate their perception of time allocated between speakers and if conversation was comprehensible. Questions relating to the second round of the survey assessed panelists' change in opinion (or lack thereof) from the first round. The cause of the change in opinion was also addressed (i.e. whether the cause was due to misunderstanding or fear of professional repercussion).

Finally, panelists were invited to provide additional comments for their experiences and opinions regarding the Modified Delphi panel. Pointed questions were asked to encourage shared opinions, such as topics that were excluded from the conversation, where this topic of research is heading, and what should be changed for future Modified Delphi panels (i.e. method of organization, conduct, etc.).

A 5-point Likert-Type scale was used to answer each question for the End of Day Survey. Responses to each question were strongly disagree, disagree, somewhat agree, agree, and strongly agree (as used in a previous similar studies (Morris et al., 2012). For analysis of results, responses were converted numerically (strongly disagree -1 , disagree -2 , somewhat agree -3 , agree -4 , strongly agree -5$)$. Numbered responses were grouped into three categories: (1) disagreement: 1 and 2,(2) uncertain: 3, and (3) agreement: 4 and 5.

\subsection{Confounders and Bias}

Certain measures were placed to reduce the risk of confounders and bias in our Modified Delphi panel. Recruitment of candidate panelists required assessment of any potential conflicts of interest. A conflict of interest form was included in the background package to ensure any potential conflicts could be addressed prior to the Delphi meeting.

The literature review provided in the background package was a potential source for bias. Including evidence from some sources while excluding it from others is damaging to the validity of the literature review. We sought to be as precise with our literature review of ASP research in nursing homes using MEDLINE and PubMed databases (see search strategy in Appendix F) 
The Modified Delphi Method utilizes discussion and encourages panelists to express their thoughts and concern (Dalkey \& Helmer, 1963). A potential confounding variable that may hinder open expression is intimidation. The expert panel consisted of individuals with broad professional representativeness from nursing home physicians and nursing home residents to ASP experts. It was likely that peer pressure could play in a role in the consensus process. To address this issue, panelists were encouraged to feel free to share any opinions. Additionally, a survey was provided to the panelists at the end of the day to assess their experience of intimidation and freedom to express opinions.

The facilitator of the Modified Delphi panel is a potential source for bias. It is arguable that the facilitator is the most important component of the panel process (Fitch et al., 2001). It is essential that the facilitator is knowledgeable in the field and is equipped to handle the panel participants without influencing their responses. 
Table 1. Summary of ASP Interventions and their Descriptions

\section{Intervention}

Antibiograms

Antibiotic

Cycling

Audit and

feedback

Clinical decision

support system

(Computerized)

De-escalation

Educational

Formulary

automatic

substitution

Formulary

restriction

Formulary review

Guidelines for

empiric

prescribing

IV to oral

transition

Multifaceted

Short course antibiotic therapy

Targeting CDI
A tool for summarizing susceptibility of bacteria to antibiotics at a given point in time.

Repeated exchange, or cycling, of antibiotics of a certain class with those of another class. Eventual return to the original antibiotic class and continued exchange.

Summarization of a clinician's prescribing performance and recommendations for adjustment.

Tool for providing clinicians with relevant information for arriving at correct diagnosis and selection of antibiotics.

Readjustment of antibiotics following culture results: transitioning from broad-spectrum to narrow-spectrum antibiotics, conversion of empiric to pathogen-directed therapy, discontinuation of unnecessary antibiotics.

Education of NH staff (nurses, physicians, HCPs, etc.) and patients/advocates to better their understanding of bacterial resistance, share knowledge of ASP principles, and improve antibiotic prescribing.

Automatic substitution of certain drugs by pharmacists without the need for prescriber consultation.

Restriction of certain antibiotics and guidance for their use due to risk of resistance/ADEs, over/misuse, broad spectrum, expense.

Institutional review of available antibiotics.

Development of standardized, multidisciplinary, evidence-based guidelines to aid prescribers in their initiation of antibiotic therapy.

Guideline implementation to transition antibiotics from IV to oral when clinically indicated.

Bundling of two or more ASP intervention strategies to form a complex intervention. Generally educational interventions combined with audit and feedback and/or guidelines. Can include physician e-learning modules, decision support, and engagement of patients.

Implementation of guidelines encouraging short durations of antibiotic therapy for specific uncomplicated infections.

Increasing appropriate therapy and adherence to guidelines for treating CDI. Includes education, guidelines, and restriction components. 


\section{Chapter 3 \\ Results}

\section{Key Objectives}

(1) To describe the Modified Delphi panel recruitment results,

(2) To present the results from Survey 1, Survey 2, including elimination of ASP interventions deemed unnecessary or irrelevant to nursing home settings, and the End of Day Survey, and

(3) To describe the resourcing necessary for implementing the appropriate and relevant ASP interventions for nursing homes and prioritization of such interventions. 


\section{Modified Delphi Panel Members}

Thirty-six candidate panel members were identified for invitation for the Modified Delphi panel. Lists containing the names and professional designations of the candidate panel members were sent to the members of the research team uninvolved with the recruitment process. Members of the research team independently reviewed candidate panel members for their professional designation, association with nursing homes, and appropriateness for inclusion in the Modified Delphi panel. 23 individuals were deemed suitable for the Modified Delphi Panel and were sent a formal invitation via email. Of the 23 individuals invited to participate in the Modified Delphi panel, $16(70 \%)$ accepted to participate (Figure 5).

Professional representativeness of the 16 panel members was diverse; most had multiple professional designations: 3 infectious disease physicians, 1 nursing home physician, 2 infection control experts, 4 ASP experts, 2 hospitalist MDs, 3 resident council representatives, 2 nurses, 4 pharmacists, 2 administration representatives, and 3 policy and stakeholder representatives (Table 2). The panel was almost evenly split female and males ( 9 female, 7 male). The national representation of the panel was less diverse than anticipated: most (11) panel members were located within the Greater Toronto Area (GTA), 3 were located within Ontario outside of the GTA, and 2 were located outside of Ontario (1 from Manitoba and 1 from Nova Scotia). 
Figure 5. Modified Delphi panel recruitment process

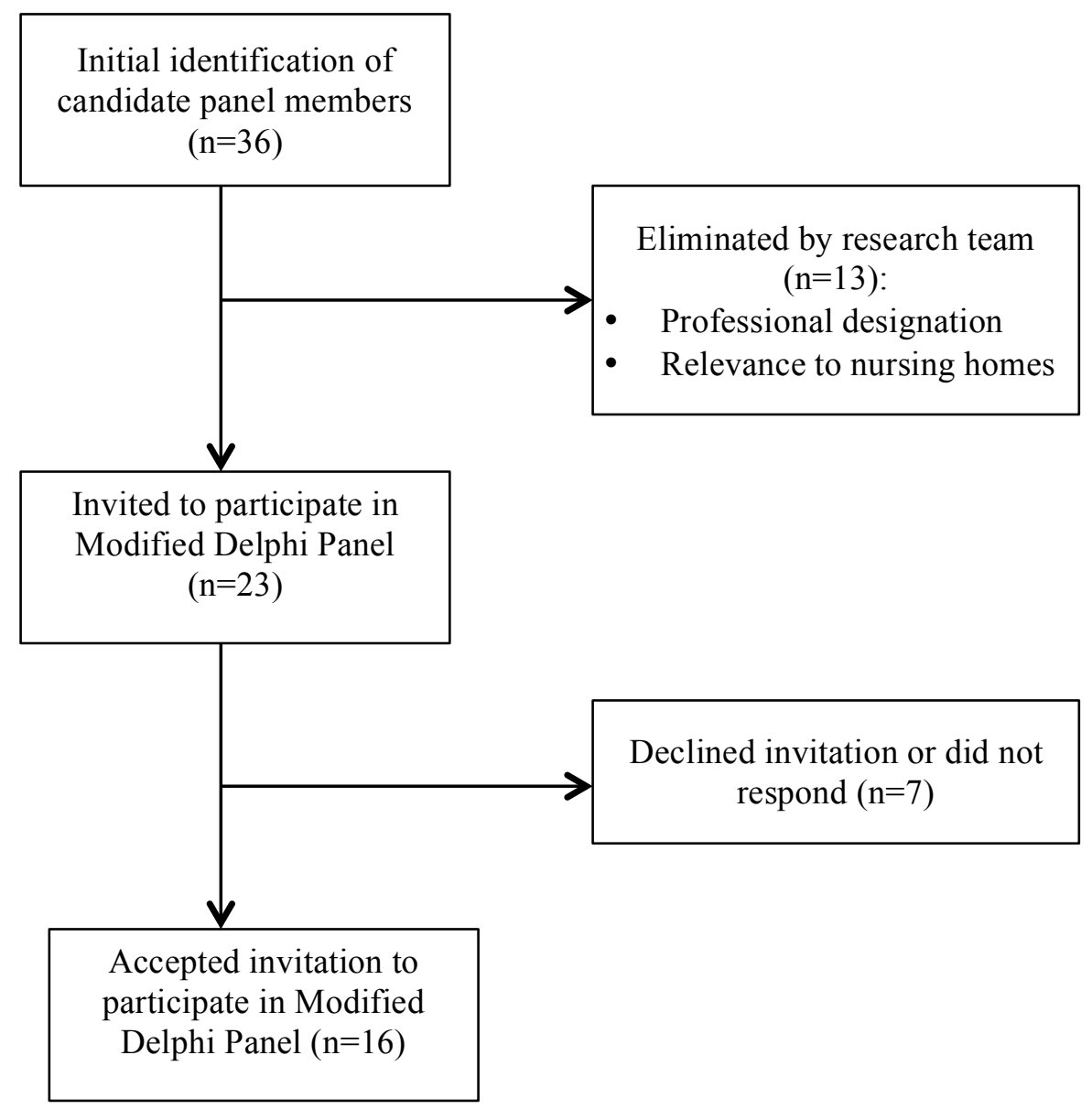


Table 2. Modified Delphi Panel Participant Representation

\begin{tabular}{|c|c|c|c|c|c|c|c|c|c|c|c|c|c|}
\hline \multirow[b]{2}{*}{ Participant } & \multicolumn{10}{|c|}{ Professional Designation } & \multicolumn{3}{|c|}{ Geographical Residence } \\
\hline & $\begin{array}{l}\text { Infectious } \\
\text { Disease }\end{array}$ & $\begin{array}{c}\text { LTC } \\
\text { Physician }\end{array}$ & $\begin{array}{c}\text { Infection } \\
\text { Control }\end{array}$ & $\begin{array}{c}\text { ASP } \\
\text { Expert }\end{array}$ & $\begin{array}{l}\text { Hospitalist } \\
\text { (MD) }\end{array}$ & $\begin{array}{c}\text { Resident/ } \\
\text { Resident } \\
\text { Council }\end{array}$ & Nursing & Pharmacy & $\begin{array}{l}\text { Admin- } \\
\text { istration }\end{array}$ & $\begin{array}{c}\text { Policy } \\
\text { Stakeholder }\end{array}$ & GTA & $\begin{array}{l}\text { Other } \\
\text { Ontario }\end{array}$ & $\begin{array}{l}\text { Other } \\
\text { Canada }\end{array}$ \\
\hline $\mathrm{P} 1^{\mathrm{a}}$ & & & $\checkmark$ & & & & $\checkmark$ & & & & & $\sqrt{ }$ & \\
\hline $\mathrm{P} 2$ & $\checkmark$ & & & & $\checkmark$ & & & & & $\checkmark$ & & & $\checkmark$ \\
\hline P3 & & & & & & & & $\checkmark$ & & & $\checkmark$ & & \\
\hline P4 & & & & & & & & $\checkmark$ & & & $\checkmark$ & & \\
\hline P5 & $\checkmark$ & & & $\checkmark$ & & & & & & & & $\checkmark$ & \\
\hline P6 & $\checkmark$ & & & $\checkmark$ & & & & & & & & & $\checkmark$ \\
\hline P7 & & & & $\checkmark$ & $\checkmark$ & & & & & & $\checkmark$ & & \\
\hline P8 & & & & & & & & & & $\checkmark$ & $\checkmark$ & & \\
\hline P9 & & & & & & & & $\checkmark$ & & & $\checkmark$ & & \\
\hline P10 & & & & & & $\checkmark$ & & & $\checkmark$ & & $\checkmark$ & & \\
\hline P11 & & & $\checkmark$ & & & & & & & & $\checkmark$ & & \\
\hline P12 & & $\checkmark$ & & & & & & & & & & $\checkmark$ & \\
\hline P13 & & & & $\checkmark$ & & & & $\checkmark$ & & $\checkmark$ & $\checkmark$ & & \\
\hline P14 & & & & & & & $\checkmark$ & & & & & $\mathcal{S}$ & \\
\hline P15 & & & & & & & & & $\checkmark$ & & $\checkmark$ & & \\
\hline $\mathrm{P} 16$ & & & & & & $\checkmark$ & & & & & & $\checkmark$ & \\
\hline
\end{tabular}

Abbreviations: LTC, long term care; ASP, antimicrobial stewardship programs; GTA, Greater Toronto Area.

${ }^{\text {a }}$ Panelist 


\section{Survey 1 Results}

The first round of the survey was sent on March 6, 2018 via email with the Background Package and Consent Form. Panelists were provided a link to the online survey, which was hosted by SimpleSurvey ${ }^{\mathrm{TM}}$. Most of the panel members completed the survey within one week after receiving the email $(15 / 16,93.8 \%$ response rate).

\subsection{Evaluative Criteria}

The first component of the survey was assessing the five evaluative criteria discussed in the previous chapter: (1) scientific merit, (2) impact, (3) feasibility, (4) accountability, and (5) importance. All of the criteria received consensus for agreement. Consensus was defined as at least $75 \%$ of the scores falling between 7-9 on the Likert-Type Scale (i.e. consensus for agreement).

Consensus was established for each of the evaluative criteria; percentages of scores between 7-9 for each criterion were $87 \%, 100 \%, 100 \%, 87 \%, 93 \%$, respectively. Comments for the assessment of evaluative criteria component of the survey were as follows:

"Not to minimize scientific merit, I think such studies will be difficult to get funding for and do, and we cannot wait for their results. I believe that we can extrapolate to a degree from the studies we have and experience in other settings (although not entirely generalizable) and make recommendations for ASPs in NH. We may even be able to identify more applicable questions and strategies for answering those questions after some ASPs implemented."

"Feasibility is the most important. It has to be easy to implement without a significant increase in resource utilization or workload."

"All of the above are most important because of the impact on the outcome of the medication used."

\subsection{Results Analysis - Adjusted}

The initial strategy for eliminating ASP interventions was for those that reached consensus for disagreement - at least $75 \%$ of scores falling between 1-3 on the Likert scale. However, no interventions attained consensus for disagreement following this method. The method was revised; criteria with scores of 7-9 falling less than 50\% were determined to be consensus for disagreement, and criteria with scores of 7-9 falling between $50-74 \%$ were determined to be 
uncertain agreement. Interventions were tabulated to display percentages of scores with agreement, uncertainty, and disagreement (example Table 3).

Interventions varied in their scores across the evaluative criteria; 9 of the 14 had mixed results, with some criteria achieving consensus for agreement, consensus for disagreement, and uncertainty. No intervention achieved consensus for agreement for all evaluative criteria but several managed to obtain consensus for disagreement across all criteria. Interventions receiving complete consensus for disagreement were reserved to discuss for elimination at the panel meeting. The following are Survey 1 results for each intervention organized from most consensus for agreement to most consensus for disagreement.

\subsubsection{Guidelines for Empiric Prescribing}

Results for guidelines for empiric prescribing interventions were mostly consensus for agreement; one criterion resulted in uncertainty: scientific merit, 80\%; impact, 87\%; feasibility, 80\%; accountability, 73\%; and importance, $87 \%$ (Table 3).

Table 3. Survey 1 Results for Guidelines for Empiric Prescribing Across Five Evaluative Criteria ${ }^{\mathrm{a}, \mathrm{b}}$

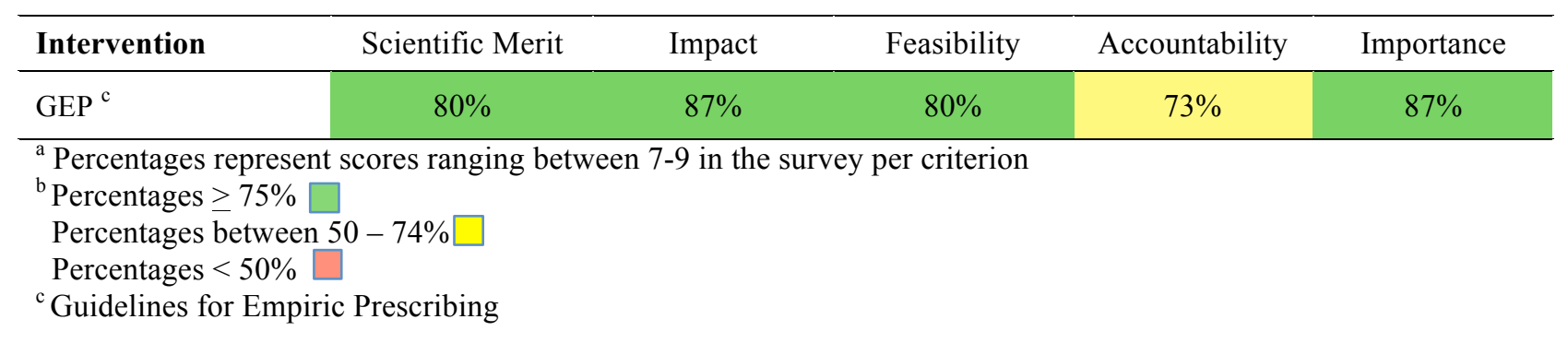

Comments for Guidelines for Empiric Prescribing:

"Important to update frequently and be made relevant."

"I see this as a bit of a clinical decision support tool. Having the guidelines is crucial. Every bit as important is a system that efficiently operationalizes following the guideline."

"This is something that could be developed by the LHIN or another authority and disseminated widely to all LTC physicians."

"When prescribing guidelines are good but not set in stone depending on adverse reactions and other diagnosis." 


\subsubsection{Short Course Antibiotic Therapy}

Results for short course antibiotic therapy interventions were mixed for consensus for agreement and uncertainty: scientific merit, 67\%; impact, 80\%; feasibility, 87\%; accountability, 73\%; and importance, $87 \%$ (Table 4).

Table 4. Survey 1 Results for Short Course Antibiotic Therapy Across Five Evaluative Criteria ${ }^{a}$ b

\begin{tabular}{lccccc}
\hline Intervention & Scientific Merit & Impact & Feasibility & Accountability & Importance \\
\hline SCAT $^{\mathrm{c}}$ & $67 \%$ & $80 \%$ & $87 \%$ & $73 \%$ & $87 \%$ \\
\hline
\end{tabular}

\footnotetext{
${ }^{\mathrm{b}}$ Percentages $\geq 75 \%$

Percentages between $50-74 \%$

Percentages $<50 \%$

${ }^{\mathrm{c}}$ Short Course Antibiotic Therapy
}

${ }^{a}$ Percentages represent scores ranging between 7-9 in the survey per criterion

Comments for Short Course Antibiotic Therapy:

"Main challenge here is a lack of literature on short course antibiotic therapy in the nursing home population. This will make it difficult to promote. Otherwise feasible if the literature is available to back it up."

"Immediate diagnosis of infection to start therapy at a low dose gives enough to lessen the impact for a better outcome."

"If RCTs [randomized controlled trials] supported short course it would have high merit" "Like for UTI - 3 day course vs 7 - 10 day course"

\subsubsection{Audit and Feedback}

The results for audit and feedback interventions were mostly uncertain but with one criterion attaining consensus for agreement: scientific merit, 60\%; impact, 87\%; feasibility, 53\%; accountability, 60\%; importance, 67\% (Table 5).

Table 5. Survey 1 Results for Audit and Feedback Across Five Evaluative Criteria ${ }^{\text {a, }}$

\begin{tabular}{lccccc}
\hline Intervention & Scientific Merit & Impact & Feasibility & Accountability & Importance \\
\hline Audit and Feedback & $60 \%$ & $87 \%$ & $53 \%$ & $60 \%$ & $67 \%$ \\
\hline
\end{tabular}

\footnotetext{
${ }^{\mathrm{a}}$ Percentages represent scores ranging between 7-9 in the survey per criterion

${ }^{\mathrm{b}}$ Percentages $\geq 75 \%$

Percentages between $50-74 \%$

Percentages $<50 \%$
} 
Comments for Audit and Feedback:

"It is crucial that the pharmacist and doctor are in agreement."

"Re accountability, our experience is that a $A \& F$ report should be more about quality important rather than use for accountability. Accountability may discourage participation into an A\&F product."

"My sense is that physicians caring for patients in NH have a high patient volume and little time. I have doubts that they will give much time to receiving feedback in this busy environment, but could well be wrong and this this should be studied."

"A LTC pharmacy should be able to gather who prescribes the most abx orders via their prescription software."

"All steps in preventing contamination and spread of bacterial or viral infections is most important in long-term care. All antibiotics prescribed will vary from one resident to another depending on what type of diagnosis each resident would have."

"Effect wanes over time."

\subsubsection{Multifaceted Interventions}

Results for multifaceted interventions were mixed for all three categories: scientific merit, 87\%; impact, 93\%; feasibility, 33\%; accountability, 40\%; and importance, 73\% (Table 6).

Table 6. Survey 1 Results for Multifaceted Interventions Across Five Evaluative Criteria ${ }^{a, b}$

\begin{tabular}{lccccc}
\hline Intervention & Scientific Merit & Impact & Feasibility & Accountability & Importance \\
\hline Multifaceted & $87 \%$ & $93 \%$ & $33 \%$ & $40 \%$ & $73 \%$ \\
\hline
\end{tabular}

\footnotetext{
${ }^{a}$ Percentages represent scores ranging between 7-9 in the survey per criterion

${ }^{\mathrm{b}}$ Percentages $\geq 75 \%$

Percentages between $50-74 \%$

Percentages $<50 \%$
}

Comments for Multifaceted Interventions:

"The more complex you get the less chance of success."

"The literature suggests multiple interventions are more effective. This makes inherent sense, in part because of different settings having different needs and, probably, different response to interventions. I think feasibility in the current financially constrained system will be a challenge to implementation."

"Combining education and guidelines might be both feasible and may have a stronger impact than each one separately."

"Multifaceted Interventions is very important to follow the improvement of the diagnosed infection for a better outcome."

"Team effort; empowerment of team players; role definitions - all required." 


\subsubsection{Clinical Decision Support Systems (CDSS)}

The results for CDSS interventions were mixed with areas of census for agreement, consensus for disagreement, and uncertainty: scientific merit, $60 \%$; impact, $87 \%$; feasibility, $47 \%$; accountability, 60\%; importance, 67\% (Table 7).

Table 7. Survey 1 Results for Clinical Decision Support Systems Across Five Evaluative Criteria ${ }^{\text {a, } b}$

\begin{tabular}{lccccc}
\hline Intervention & Scientific Merit & Impact & Feasibility & Accountability & Importance \\
\hline CDSS & $60 \%$ & $87 \%$ & $47 \%$ & $60 \%$ & $67 \%$ \\
\hline
\end{tabular}

\footnotetext{
${ }^{\text {a }}$ Percentages represent scores ranging between 7-9 in the survey per criterion

${ }^{\mathrm{b}}$ Percentages $\geq 75 \%$

Percentages between $50-74 \%$

Percentages $<50 \%$
}

Comments for CDSS:

"I think all physicians want to do the right thing and have reliable supports to assist them in this goal. As such, I think clinical decision support systems are the way to go. I do have questions about whether NH will financially support purchase and use of such systems (I suspect they will be computer based and not free)."

"Though good to have, in the current state of LTC (where some homes are still paper charts, and MD's write prescriptions by hand) it doesn't seem very feasible."

"Any diagnosis and prescribing accordingly is most important in long-term care to prevent any resident from becoming septic at the point of end of life."

"At this stage, physicians are still doing paper-based ordering. In the future, with PrescribeIT, this has some potential."

\subsubsection{De-escalation}

The results for de-escalation interventions were uncertain for all evaluative criteria: scientific merit, 67\%; impact, 67\%; feasibility, 53\%; accountability, 53\%; and importance, 67\% (Table 8).

Table 8. Survey 1 Results for De-escalation Across Five Evaluative Criteria ${ }^{\text {a, }}$

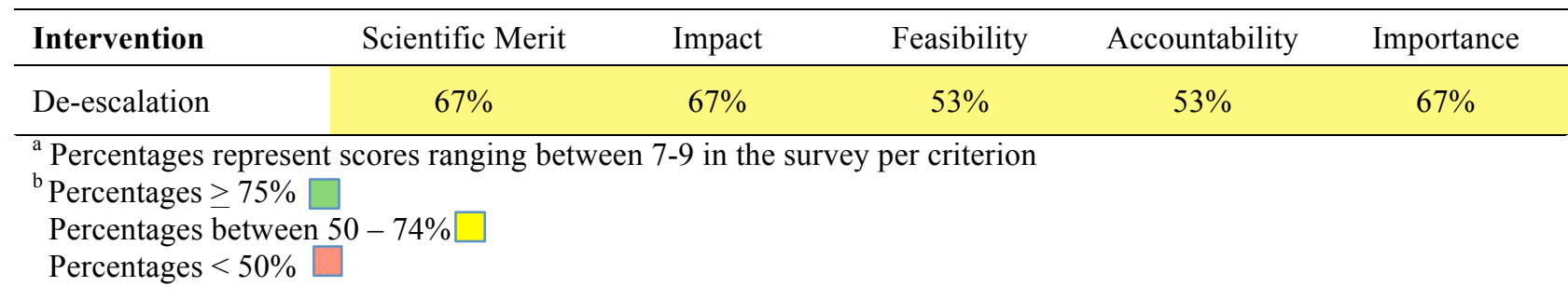

Comments for De-Escalation: 
"I think this is very important. My question (hence the low feasibility rating) is who will do it? Will they get the cultures?"

"Use of cultures to guide therapy is already in practice for UTIs, but URTI are commonly treated empirically (hard to get respiratory cultures) from my understanding . To have someone tracking each antibiotic prescription for appropriate length of therapy would require a brand new role and possibly a lot of resources."

"Proper monitoring and testing is important for proper follow-up of diagnosis to end treatment."

"Does not address empiric use."

"Pharmacists could be a big player in implementing this strategy; funding model has to fit."

\subsubsection{Targeting CDI}

Results for interventions targeting CDI were all uncertain: scientific merit, 60\%; impact, 60\%; feasibility, 60\%; accountability, 53\%; and importance, 60\% (Table 9).

Table 9. Survey 1 Results for Targeting CDI Across Five Evaluative Criteria ${ }^{\text {a, }}$

\begin{tabular}{lccccc}
\hline Intervention & Scientific Merit & Impact & Feasibility & Accountability & Importance \\
\hline Targeting CDI & $60 \%$ & $60 \%$ & $60 \%$ & $53 \%$ & $60 \%$ \\
\hline
\end{tabular}

${ }^{\mathrm{a}}$ Percentages represent scores ranging between 7-9 in the survey per criterion

${ }^{\mathrm{b}}$ Percentages $\geq 75 \%$

Percentages between $50-74 \%$

Percentages $<50 \%$

Comments for Targeting CDI:

"Focus on prevention (e.g., agents with higher propensity for CDI may be more important)"

"Personally don't see a lot of CDI in nursing homes. Unclear how relevant it would be compared to UTI or URTIs"

"Adherence to guidelines for both treating the CDI needs ongoing education, proper prescribing, sanitizing constantly and restricting components is most urgent to have a good outcome."

"Drug coverage will be a concern here; government medication formularies must be kept current"

"Some interventions for CDI are unrealistic for some LTC homes i.e. sanitizing a room $2 x$ day - staff resistance to do the work, lack of staff to do the work" 


\subsubsection{Antibiograms}

Antibiogram interventions were associated with uncertainty but received consensus for disagreement for one of the criteria: scientific merit, $53 \%$; impact, $60 \%$; feasibility, $53 \%$; accountability, 47\%; and importance, 60\% (Table 10).

Table 10. Survey 1 Results for Antibiograms Across Five Evaluative Criteria ${ }^{\text {a, }}$

\begin{tabular}{lccccc}
\hline Intervention & Scientific Merit & Impact & Feasibility & Accountability & Importance \\
\hline Antibiograms & $53 \%$ & $60 \%$ & $53 \%$ & $47 \%$ & $60 \%$ \\
\hline
\end{tabular}

\footnotetext{
${ }^{a}$ Percentages represent scores ranging between $7-9$ in the survey per criterion

${ }^{\mathrm{b}}$ Percentages $\geq 75 \%$

Percentages between $50-74 \%$

Percentages $<50 \%$
}

Comments for Antibiograms:

"My hunch is that physicians in NH are unlikely to refer to this when making antimicrobial choices. I wonder if it could even drive broader antimicrobial use. For that reason I think it would be worth studying and using it for accountability purposes"

"This intervention would be important to help form the basis of guidelines for selecting antibiotics for a particular facility."

"If proper tools are not used the outcome could become drastic and very contagious"

\subsubsection{Educational Interventions}

The results for educational interventions were mostly uncertain but with one criterion achieving consensus for disagreement: scientific merit, $60 \%$; impact, $67 \%$; feasibility, $67 \%$; accountability, 47\%; and importance, $73 \%$ (Table 11).

Table 11. Survey 1 Results for Education Across Five Evaluative Criteria ${ }^{a, b}$

\begin{tabular}{|c|c|c|c|c|c|}
\hline Intervention & Scientific Merit & Impact & Feasibility & Accountability & Importance \\
\hline Education & $60 \%$ & $67 \%$ & $67 \%$ & $47 \%$ & $73 \%$ \\
\hline
\end{tabular}

Comments for Education: 
"In a long-term care home setting the RPNs along with the RNs and front line workers are aware of what's involved as they see the residents 24/7 and they are the folks that have the closest relationships to the residents - they can spot physical changes before anyone else - this is very important."

"Potential high impact and most well-studied in LTC, but usually multi-faceted interventions. Should be used in combination with other strategies."

"Hate to be negative, but education is not a greater sustainer of change. Don't we have enough data, at least in other settings, that it really does not effect long-term change in most behaviours?"

"Feasible to do education. However, my understanding is that evidence it really had a big impact is poor. Patient and family member education is a good thing as sometimes they are the driving forces for overuse of antibiotics."

"Education is very important in long-term care and needs to be ongoing learning for better outcomes.”

"Education is always a good strategy in combination with other process changes (e.g., checklists, forcing functions). Rarely does education alone change behaviours."

"In a LTC setting, education will be key to informing residents and family members of the reasons why an antibiotic is not prescribed, where in their lived experience, it has been prescribed in the past. It will be important to explain the negative outcomes associated with overuse, prescribing when there is not scientific evidence to do so-sometimes people have an emotional/psychological attachment to receiving prescription medication for illness even if it's not only not going to do good, but actually do harm."

\subsubsection{Formulary Restriction}

The results for formulary restriction interventions were mostly consensus for disagreement excepting one criterion, which scored for uncertainty: scientific merit, 47\%; impact, $67 \%$; feasibility, 40\%; accountability, 47\%; and importance, 47\% (Table 12).

Table 12. Survey 1 Results for Formulary Restriction Across Five Evaluative Criteria ${ }^{\text {a b }}$

\begin{tabular}{lccccc}
\hline Intervention & Scientific Merit & Impact & Feasibility & Accountability & Importance \\
\hline Formulary Restriction & $47 \%$ & $67 \%$ & $40 \%$ & $47 \%$ & $47 \%$ \\
\hline${ }^{a}$ Percentages represent scores ranging between $7-9$ in the survey per criterion & \\
${ }^{b}$ Percentages $\geq 75 \% \square$ \\
Percentages between $50-74 \% \square$ \\
Percentages $<50 \% \square$ \\
Comments for Formulary Restriction: \\
"Clinicians should use the best tool and not be influenced by the pharmaceutical \\
companies who are trying to promote their products."
\end{tabular}


"Restrictions are hard to enforce, and hence lose effectiveness. Guidance needs an implementation strategy to be effective. On their own, don't see that they will be particularly effective."

"Perhaps can be implemented in some of the larger corporate chains after extensive discussion with medical staff. But I foresee difficulties with this. Pharmacy may struggle as an LTC pharmacy looks after many homes which belong to different chains so staff will struggle to enforce these restrictions."

"Risk of resistance, adverse drug events, and over use of prescriptions could cause a restriction which states that the intervention would be important and could cause quite an impact."

"More effective in a hospital setting when IV antibiotics are being prescribed. ODB formulary already has some restrictions - though override codes are often used inappropriately. The process to contact the prescriber to get changes made or approved is cumbersome, often leading to delay in therapy."

\subsubsection{Antibiotic Cycling}

Antibiotic cycling interventions received consensus for disagreement across all evaluative criteria: scientific merit, 20\%; impact, 13\%; feasibility, 20\%; accountability, 13\%; and importance, $13 \%$ (Table 13).

Table 13. Survey 1 Results for Antibiotic Cycling Across Five Evaluative Criteria ${ }^{a} b$

\begin{tabular}{|c|c|c|c|c|c|}
\hline Intervention & Scientific Merit & Impact & Feasibility & Accountability & Importance \\
\hline Antibiotic Cycling & $20 \%$ & $13 \%$ & $20 \%$ & $13 \%$ & $13 \%$ \\
\hline $\begin{array}{l}{ }^{\mathrm{a}} \text { Percentages repre } \\
\mathrm{b}^{\mathrm{b}} \text { Percentages } \geq 75^{\circ} \\
\text { Percentages betwe } \\
\text { Percentages }<50^{\circ}\end{array}$ & $\begin{array}{l}\text { scores ranging bety } \\
0-74 \% \square\end{array}$ & $7-9$ in the & per criterion & & \\
\hline \multicolumn{6}{|c|}{ Comments for Antibiotic Cycling: } \\
\hline \multicolumn{6}{|c|}{ "It is important to limit antimicrobial use, not just change it." } \\
\hline \multicolumn{6}{|c|}{$\begin{array}{l}\text { "Although I understand that one article suggested this as an effective intervention, the } \\
\text { literature in general has not supported cycling as an effective intervention. I don't think it } \\
\text { is a high yield strategy and would not prioritize it highly." }\end{array}$} \\
\hline \multicolumn{6}{|c|}{$\begin{array}{l}\text { "May not be very feasible to do this in multiple facilities with many different staff, } \\
\text { cultures etc." }\end{array}$} \\
\hline
\end{tabular}




\subsubsection{Formulary Automatic Substitution}

Formulary automatic substitution interventions received consensus for disagreement across all evaluative criteria: scientific merit, 22\%; impact, 33\%; feasibility, 33\%; accountability, 20\%; and importance, $20 \%$ (Table 14).

Table 14. Survey 1 Results for Formulary Automatic Substitution Across Five Evaluative Criteria ${ }^{\text {a b }}$

\begin{tabular}{|c|c|c|c|c|c|}
\hline Intervention & Scientific Merit & Impact & Feasibility & Accountability & Importance \\
\hline FAS $^{\mathrm{c}}$ & $22 \%$ & $33 \%$ & $33 \%$ & $20 \%$ & $20 \%$ \\
\hline
\end{tabular}

Comments for Formulary Automatic Substitution:

"It's too risky - it's important to have consultation. Where's the trust? You have to know the person and trust the person to do what they've said - prescribed."

"How many NH have enough pharmacists to do this? Will they be repeatedly overrided?"

"Too many players to make this very feasible (i.e. too many physicians in too many different facilities including on-call physicians who may not be familiar with this autosub policy)”

"Prescribing needs constant monitoring to ensure that there is no adverse interaction."

"Will only work if the pharmacist is included in the team and has access to necessary information to make a clinical decision." 


\title{
12.2.13 Formulary Review
}

Formulary review interventions received consensus for disagreement across all of the evaluative criteria: scientific merit, 33\%; impact, 40\%; feasibility, 47\%; accountability, 33\%; and importance, $47 \%$ (Table 15).

Table 15. Survey 1 Results for Formulary Review Across Five Evaluative Criteria ${ }^{\text {a, }}$

\begin{tabular}{lccccc}
\hline Intervention & Scientific Merit & Impact & Feasibility & Accountability & Importance \\
\hline Formulary Review & $33 \%$ & $40 \%$ & $47 \%$ & $33 \%$ & $47 \%$ \\
\hline
\end{tabular}

\footnotetext{
${ }^{a}$ Percentages represent scores ranging between $7-9$ in the survey per criterion

${ }^{\mathrm{b}}$ Percentages $\geq 75 \%$

Percentages between $50-74 \%$

Percentages $<50 \%$
}

Comments for Formulary Restriction:

\begin{abstract}
"Crucial"
"Important to avoid duplication, just not convinced that it will make a difference in terms of inappropriate use."

"Sceptical that this is going to have a meaningful impact on antibiotic resistance."

"The right prescription may be different for each person and needs to be prescribed accordingly to diagnosis and other diagnosis to be aware of adverse reactions."

"Most physicians covering patients in nursing homes, being family physicians are not used to the formulary system and its limitations. This would be a practice change - and enforcing it would be a challenge. Who would enforce it?"
\end{abstract}

\subsubsection{IV to Oral Transition}

Results for IV to oral transition interventions were consensus for disagreement across all criteria: scientific merit, 33\%; impact, 40\%; feasibility, 40\%; accountability, 40\%; and importance, 27\% (Table 16).

Table 16. Survey 1 Results for IV to Oral Transition Across Five Evaluative Criteria a, b

\begin{tabular}{lccccc}
\hline Intervention & Scientific Merit & Impact & Feasibility & Accountability & Importance \\
\hline IV to Oral Transition & $33 \%$ & $40 \%$ & $40 \%$ & $40 \%$ & $27 \%$ \\
\hline${ }^{\text {a }}$ Percentages represent scores ranging between $7-9$ in the survey per criterion & & \\
${ }^{\mathrm{b}}$ Percentages $\geq 75 \% \square$ \\
Percentages between $50-74 \% \square$ \\
Percentages $<50 \% \square$
\end{tabular}


Comments for IV to Oral Transition:

"I don't know the Ontario situation, but am presuming that a relatively small proportion of patients is on IV antibiotics and, as such, this will not have a big impact on use."

"Nursing home residents are rarely on IV antibiotics (as outpatient IV usually has to be arranged through CCAC, most nursing staff not used to doing IV). Typically if they are receiving outpatient IV antibiotics there usually is a good reason for it (e.g. osteomyelitis infection, endocarditis etc.). Rarely seen overall."

"Guidelines implementation to transition antibiotics from IV to oral is part of the monitoring of the prescription to a better outcome."

"With over 65\% of residents in LTC homes living with dementia, taking medication orally can be very difficult."

\subsection{Other Comments}

Panelists were invited to provide final comments following evaluation of the interventions, such as for interventions that were not included in the survey:

"We should not overlook the value and use of alternative medicine from an acknowledged profession - I used herbal alternative medication to reduce the intensity and duration of shingles and pneumonia"

"Communication/assessment tools. Some have been studied to help ensure appropriate resident evaluation for those with suspected infection. Since prescriber is offsite, nurse communication of resident assessment is very important."

"Discussion of good guidelines and resources that one can being to implement right now."

"I feel the importance of ongoing education in long-term care is so very important for quality of life in long-term care for residents, staff and families and volunteers. Anyone visiting or working in long-term care. Proper sanitization of hands, hand rails, and any equipment or tools that may be constantly handled at all times." 


\section{Panel Day}

\subsection{Survey 1 Review and Intervention Elimination Phase 1}

Following introductions and a brief background discussion, panelists were guided to review the Survey 1 results document (Appendix G). Attention was directed at ASP interventions receiving consensus for disagreement across all the evaluative criteria: antibiotic cycling, formulary automatic substitution, formulary review, and IV to oral transition. The facilitator explained that these interventions were to be eliminated due to their low scores from the first survey but opened the floor to discussion. All of interventions receiving consensus for disagreement were eliminated; however, discussion grew around Formulary Automatic Substitution interventions, which resulted in a decision to reserve the intervention for further discussion and Survey 2 (see comments below).

"Why did Formulary Automatic Substitution score so low? The system should be feasible for its inclusion."

"Pharmacies do not have their own formularies, would not be effective ... people tricking the system"

The remaining interventions were addressed in the order they were included in Survey 1.

Discussion for each intervention concluded in completion of Survey 2. The results for Survey 2 determined the inclusion of the intervention for resource discussion and prioritization. 


\subsection{Survey 2 and Intervention Elimination Phase 2}

\subsubsection{Guidelines for Empiric Prescribing}

Guidelines for empiric prescribing received consensus for agreement across all criteria: scientific merit, 75\%; impact, 81\%; feasibility, 88\%; accountability, $81 \%$; and importance, $100 \%$ (Table 17).

Table 17. Survey 2 Results for Guidelines for Empiric Prescribing Across Five Evaluative Criteria a, b, c

\begin{tabular}{lccccc}
\hline Intervention & Scientific Merit & Impact & Feasibility & Accountability & Importance \\
\hline $\mathrm{GEP}^{\mathrm{c}}$ & $75 \%$ & $81 \%$ & $88 \%$ & $81 \%$ & $100 \%$ \\
\hline
\end{tabular}

\footnotetext{
${ }^{\mathrm{b}}$ Percentages $\geq 75 \%$

Percentages between $50-74 \%$

Percentages $<50 \%$

${ }^{\mathrm{c}}$ Guidelines for Empiric Prescribing
}

${ }^{a}$ Percentages represent scores ranging between 7-9 in the survey per criterion

Discussion for Guidelines for Empiric Prescribing:

"Absolutely essential to have physicians involved in development of guidelines"

"Sometimes guidelines do more harm than good"

"NBI - needed but insufficient"

\subsubsection{Audit and Feedback}

Audit and feedback received greater consensus for agreement for Survey 2 than Survey 1: scientific merit, 100\%; impact, 81\%; feasibility, $69 \%$; accountability, $81 \%$; and importance, $88 \%$ (Table 18).

Table 18. Survey 2 Results for Audit and Feedback Across Five Evaluative Criteria ${ }^{a, b}$

\begin{tabular}{lccccc}
\hline Intervention & Scientific Merit & Impact & Feasibility & Accountability & Importance \\
\hline Audit and Feedback & $100 \%$ & $81 \%$ & $69 \%$ & $81 \%$ & $88 \%$ \\
\hline${ }^{\mathrm{a}}$ Percentages represent scores ranging between $7-9$ in the survey per criterion & & & \\
${ }^{\mathrm{b}}$ Percentages $\geq 75 \% \square$ & & & & \\
Percentages between $50-74 \% \square$ & & & \\
Percentages $<50 \% \square$ & & &
\end{tabular}

"First step is studying prescription patterns - study by prescriber, then feedback" "Studies show A\&F has high scientific merit. Has been well studied and shown to be beneficial; however, feasibility is an issue." 
"Skeptical about long-term sustainability. Lots of diagnostic uncertainty. Need more point-of-care testing”

\subsubsection{Communication Tools}

Communication Tools was unintentionally neglected from Survey 1: scientific merit, 56\%; impact, 75\%; feasibility, 94\%; accountability, 94\%; and importance, 88\% (Table 19).

Table 19. Survey 2 Results for Communication Tools Across Five Evaluative Criteria ${ }^{\text {a, }}$

\begin{tabular}{lccccc}
\hline Intervention & Scientific Merit & Impact & Feasibility & Accountability & Importance \\
\hline Communication Tools & $56 \%$ & $75 \%$ & $94 \%$ & $94 \%$ & $88 \%$ \\
\hline${ }^{\mathrm{a}}$ Percentages represent scores ranging between $7-9$ in the survey per criterion & & & \\
${ }^{\mathrm{b}}$ Percentages $\geq 75 \%$ \\
Percentages between $50-74 \% \square$ \\
Percentages $<50 \%$
\end{tabular}

Discussion for Communication Tools:

"This could be very beneficial to both home medical team, and family and resident."

"Easy to implement in LTC - can be paper-based, then easily integrated into technology solutions."

"This seems to me to be the same or very similar to structured communication forms and I'm not going to want to just go ahead with this in our home"

\subsubsection{De-Escalation (Renamed Scheduled Antibiotic Reassessment)}

Renamed "Scheduled Antibiotic Reassessment"; received greater consensus for agreement following Survey 2: scientific merit, 88\%; impact, 100\%; feasibility, 63\%; accountability, 94\%; and importance, $100 \%$ (Table 20).

Table 20. Survey 2 Results for Scheduled Antibiotic Reassessment Across Five Evaluative Criteria ${ }^{\text {a, b, c }}$

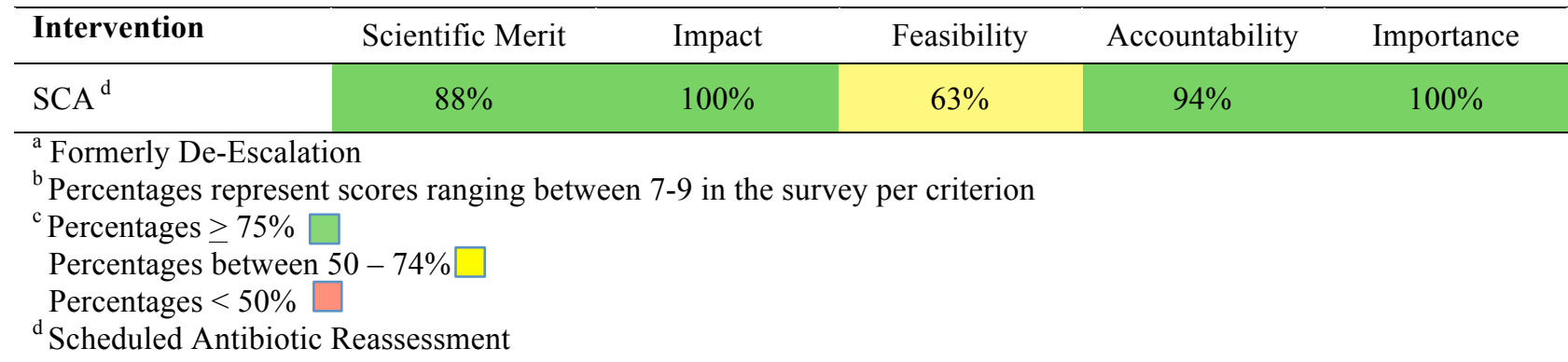


Discussion for Scheduled Antibiotic Reassessment:

"In LTC, prescribers often never sees patient and/or culture results"

"Need structured approach for reassessment; need to include in Policy and Procedures; need to know indication of start of antibiotics"

\subsubsection{Short Course Antibiotic Therapy}

Short Course Antibiotic Therapy received consensus for disagreement for scientific merit and consensus for agreement for accountability (unlike Survey 1) following Survey 2: scientific merit, 38\%; impact, 94\%; feasibility, 100\%; accountability, 94\%; and importance, $88 \%$ (Table 21).

Table 21. Survey 2 Results for Short Course Antibiotic Therapy Across Five Evaluative Criteria a, b

\begin{tabular}{lccccc}
\hline Intervention & Scientific Merit & Impact & Feasibility & Accountability & Importance \\
\hline SCAT $^{\mathrm{c}}$ & $38 \%$ & $94 \%$ & $100 \%$ & $94 \%$ & $88 \%$ \\
\hline${ }^{\mathrm{a}} \mathrm{Per}^{2}$ & & & & $94 \%$ & \\
\hline
\end{tabular}

\footnotetext{
${ }^{\mathrm{b}}$ Percentages $\geq 75 \%$

Percentages between $50-74 \%$

Percentages $<50 \%$

${ }^{\mathrm{c}}$ Short Course Antibiotic Therapy
}

${ }^{\mathrm{a}}$ Percentages represent scores ranging between 7-9 in the survey per criterion

Discussion for Short Course Antibiotic Therapy:

"If we can get some experts to endorse this, in back of evidence, to make prescribers feel this will work. Needs to be part of facility's Policy and Procedures"

"Education is strongly linked to this because residents and family members often feel that finishing the entire long round of antibiotics is necessary"

"RCTs would be worthwhile"

"I am comfortable extrapolating from other populations to say high scientific merit" 


\subsubsection{Clinical Decision Support Systems}

Clinical decision support systems received greater consensus for agreement following Survey 2: scientific merit, 56\%; impact, 94\%; feasibility, 38\%; accountability, 81\%; and importance, $75 \%$ (Table 22).

Table 22. Survey 2 Results for Clinical Decision Support Systems Across Five Evaluative Criteria ${ }^{\text {a, } b}$

\begin{tabular}{lccccc}
\hline Intervention & Scientific Merit & Impact & Feasibility & Accountability & Importance \\
\hline CDSS $^{\mathrm{c}}$ & $56 \%$ & $94 \%$ & $38 \%$ & $81 \%$ & $75 \%$ \\
\hline
\end{tabular}

${ }^{\mathrm{a}}$ Percentages represent scores ranging between $7-9$ in the surv
${ }^{\mathrm{b}}$ Percentages $\geq 75 \%$
Percentages between $50-74 \% \square$
Percentages $<50 \% \square$
${ }^{\mathrm{c}}$ Clinical Decision Support Systems
Discussion for Clinical Decision Support Systems:

"MOHLTC funding would be absolutely necessary for this"

"Hugely feasible once the ideal connected and user friendly EMR is finally implemented"

"I think this will ultimately be crucial; today, feasibility is a major stumbling block"

\subsubsection{Education}

Education received greater consensus for disagreement following Survey 2 and was eliminated from discussing resourcing and prioritization: scientific merit, 19\%; impact, 25\%; feasibility, 69\%; accountability, $31 \%$; and importance, $44 \%$ (Table 23 ).

Table 23. Survey 2 Results for Education Across Five Evaluative Criteria ${ }^{a, b}$

\begin{tabular}{|c|c|c|c|c|c|}
\hline Intervention & Scientific Merit & Impact & Feasibility & Accountability & Importance \\
\hline Education & $19 \%$ & $25 \%$ & $69 \%$ & $31 \%$ & $44 \%$ \\
\hline $\begin{array}{l}{ }^{\mathrm{a}} \text { Percentages } \mathrm{r} \\
{ }^{\mathrm{b}} \text { Percentages } \\
\text { Percentages } \mathrm{b} \\
\text { Percentages }\end{array}$ & $\begin{array}{l}\text { cores ranging bet } \\
-74 \% \square\end{array}$ & $7-9$ in the & per criterion & & \\
\hline \multicolumn{6}{|c|}{ Discussion for Education: } \\
\hline \multicolumn{6}{|c|}{ "General education is necessary but insufficient (NBI)" } \\
\hline \multicolumn{6}{|c|}{ "Does not work when done alone for health care workers" } \\
\hline “Edr & elated to spect & erventi & l be need & & \\
\hline
\end{tabular}




\subsubsection{Antibiograms}

Antibiograms received consensus for disagreement across all criteria for Survey 2 and was eliminated from discussing resourcing and prioritization: scientific merit, $6 \%$; impact, $6 \%$; feasibility, $6 \%$; accountability, $6 \%$; and importance, $6 \%$ (Table 24 ).

Table 24. Survey 2 Results for Antibiograms Across Five Evaluative Criteria ${ }^{\text {a, }}$

\begin{tabular}{lccccc}
\hline Intervention & Scientific Merit & Impact & Feasibility & Accountability & Importance \\
\hline Antibiograms & $6 \%$ & $6 \%$ & $6 \%$ & $6 \%$ & $6 \%$ \\
\hline
\end{tabular}

\footnotetext{
${ }^{a}$ Percentages represent scores ranging between 7-9 in the survey per criterion

${ }^{\mathrm{b}}$ Percentages $\geq 75 \%$

Percentages between $50-74 \%$

Percentages $<50 \%$

Discussion for Antibiograms:
}
"Infection trends and antibiotic usage are reported in quarterly reports; no indication data”

"Three most common infections in NHs: SSTI, RTI, UTI. Antibiograms only help with UTIs, since SSTIs and RTIs do not have cultures"

\subsubsection{Formulary Automatic Substitution}

Formulary automatic substitution received consensus for disagreement across all criteria following Survey 2 and was eliminated from discussing resourcing and prioritization: scientific merit, 25\%; impact, 6\%; feasibility, 0\%; accountability, 13\%; and importance, 13\% (Table 25).

Table 25. Survey 2 Results for Formulary Automatic Substitution Across Five Evaluative Criteria a, b

\begin{tabular}{lccccc}
\hline Intervention & Scientific Merit & Impact & Feasibility & Accountability & Importance \\
\hline FAS $^{\mathrm{c}}$ & $25 \%$ & $6 \%$ & $0 \%$ & $13 \%$ & $13 \%$ \\
\hline
\end{tabular}

${ }^{a}$ Percentages represent scores ranging between 7-9 in the survey per criterion

${ }^{\mathrm{b}}$ Percentages $\geq 75 \%$

Percentages between $50-74 \%$

Percentages $<50 \%$

${ }^{\mathrm{c}}$ Formulary Automatic Substitution

Discussion for Formulary Automatic Substitution:

"Generic substitutions are done as an automatic substitution - can this be expanded to appropriate prescribing?"

"If you have guidelines that everyone adheres to, then this isn't necessary"

"Done in acute care as a medical directive - not feasible in LTC" 


\subsubsection{Formulary Restriction}

Formulary restriction received consensus for disagreement across all criteria following Survey 2 and was eliminated from discussing resourcing and prioritization: scientific merit, 19\%; impact, $31 \%$; feasibility, $6 \%$; accountability, $25 \%$; and importance, $13 \%$ (Table 26 ).

Table 26. Survey 2 Results for Formulary Restriction Across Five Evaluative Criteria ${ }^{a, b}$

\begin{tabular}{lccccc}
\hline Intervention & Scientific Merit & Impact & Feasibility & Accountability & Importance \\
\hline Formulary Restriction & $19 \%$ & $31 \%$ & $6 \%$ & $25 \%$ & $13 \%$ \\
\hline
\end{tabular}

\footnotetext{
${ }^{a}$ Percentages represent scores ranging between 7-9 in the survey per criterion

${ }^{\mathrm{b}}$ Percentages $\geq 75 \%$

Percentages between $50-74 \%$

Percentages $<50 \%$
}

Discussion for Formulary Restriction:

"Could have huge impact but would need to be implemented provincially by Ontario Drug Benefit Program"

\subsubsection{Targeting CDI}

Interventions for Targeting CDI received consensus for disagreement across all criteria: scientific merit, $31 \%$; impact, $13 \%$; feasibility, $13 \%$; accountability, $13 \%$; importance $25 \%$ (Table 27 ).

Table 27. Survey 2 Results for Targeting CDI Across Five Evaluative Criteria ${ }^{\text {a, }}$

\begin{tabular}{lccccc}
\hline Intervention & Scientific Merit & Impact & Feasibility & Accountability & Importance \\
\hline Targeting CDI & $31 \%$ & $13 \%$ & $13 \%$ & $13 \%$ & $25 \%$ \\
\hline${ }^{\mathrm{a}}$ Percentages represent scores ranging between $7-9$ in the survey per criterion & & & \\
${ }^{\mathrm{b}}$ Percentages $\geq 75 \% \square$ & & & \\
Percentages between $50-74 \% \square$ & & & \\
Percentages $<50 \% \square$ & & &
\end{tabular}

"Other interventions will address this. Good antibiotic use will automatically result in reduced CDI occurrences"

"Focus on prevention - which is covered by other interventions" 


\subsubsection{Multifaceted Interventions}

Prior to completing Survey 2, the panel came to a consensus that multifaceted interventions were necessary and likely an effective intervention; however, due to feasibility concerns, implementation in nursing homes would be impossible. Therefore, multifaceted interventions were eliminated from Survey 2 and further discussion.

\subsection{Resourcing}

Of the original 14 ASP interventions (plus the addition of Communication Tools), only 6 remained for discussing resourcing and prioritization. This section will describe the resources suggested by panel members for the 6 interventions deemed necessary and essential for nursing homes.

\subsubsection{Audit and Feedback}

"Provincial report is essential - an EMR capturing patient-level prescriptions."

"Need some form of central data repository."

"Team for creating reports."

"Report on antibiotics as part of public reporting of LTC quality."

\subsubsection{Clinical Decision Support Systems}

"Implementing EMR in LTC is hugely necessary."

"Some overlap with guidelines."

\subsubsection{Scheduled Reassessment of Antibiotics}

"EMR - long-term solution."

"SBAR [situation, background, assessment, recommendation] - add at 3-day review."

"May need more nursing time."

"Continuity of care is a big issue. Staffing issues. Very difficult to do with nursing home staffing. Staffing not keeping up with change. Looks like another duty."

"Need to change the system. Need funding from MOH to make this happen."

\subsubsection{Guidelines for Empiric Prescribing}

"CPG [Clinical Practice Guidelines] team. Lead MD in LTC would be part of the team. Nurse managers. ID specialists. Residents." 
"Look at existing guidelines and see what would apply"

"HQO is currently looking into this - synthesizing existing guidelines into simple statements"

"Provincial working group to guide local areas using antibiograms"

"Centralized process to house guidelines, then distribute them and assess their usefulness at the local level"

"A group tasked with summarizing current guidelines and deciding which are applicable to LTC"

\subsubsection{Short Course Antibiotic Therapy}

"Lack of scientific merit - need to develop evidence base"

"Legislative support for shortening length of therapy"

"Electronic prescriptions for LTC"

"Medical directives, automatic stop dates for specific indications"

\subsubsection{Communication Tools}

"Guideline developed by SHEA is compatible with this"

"Multipronged approach"

"Need more resources and funding allocated to ASPs"

\subsection{Prioritization}

The final 6 ASP interventions were prioritized for necessity and relevance to nursing homes; each intervention was ranked by a number from 1-6. Posters (one per intervention) were placed around the room and panelists were provided time to place a ranking number for each intervention. Scores for each intervention were tabulated and mean scores were compared (Table 28).

Guidelines for empiric prescribing received the highest priority score (mean score of 1.3). Audit and feedback and communication tools tied for second highest priority (mean score of 3.4). Short course antibiotic therapy received third priority (mean score of 3.9). Scheduled antibiotic reassessment received fourth priority (mean score of 4.4). Clinical decision support systems received fifth priority (mean score of 4.6). 
Table 28. Prioritization of ASP Interventions for Nursing Homes

\begin{tabular}{llc}
\hline Interventions & Raw Score & Mean \\
\hline Guidelines for Empiric Prescribing & $1,1,1,1,1,1,1,1,1,1,1,1,1,3,4,1$ & $\mathbf{1 . 3}$ \\
Audit and Feedback & $3,3,4,2,1,3,3,3,4,5,6,5,5,4,2,2$ & $\mathbf{3 . 4}$ \\
Communication Tools & $2,2,4,6,5,6,4,3,2,3,1,3,4,2,4,4$, & $\mathbf{3 . 4}$ \\
Short Course Antibiotic Therapy & $2,2,4,5,2,2,6,6,6,3,3,2,2,5,6,6$ & $\mathbf{3 . 9}$ \\
$\begin{array}{l}\text { Scheduled Antibiotic Reassessment } \\
\text { (formerly De-escalation) }\end{array}$ & $4,2,5,3,5,5,5,6,5,3,6,3,2,6,5,5$ & $\mathbf{4 . 4}$ \\
Clinical Decision Support Systems & $6,6,5,4,6,3,4,4,6,5,5,6,2,4,4,3$ & $\mathbf{4 . 6}$ \\
\hline
\end{tabular}

${ }^{\mathrm{a}}$ Panelists instructed to rank interventions from 1-6

\subsection{End of Day Survey}

Panelists were invited to complete an End of Day survey to summarize their experience throughout the Modified Delphi Process (Table 29). The survey reviewed experiences from before and during the Delphi panel meeting. Pre-meeting questions addressed the background document, instructions for Survey 1, and expectations for the panel meeting. Questions referring to the panel meeting included feelings of free expression of opinion, feeling respected, heard, fair time allowance for each panelist, and easy-to-follow conversation. Panelists were asked three questions referring to Survey 2: if intimidation caused opinions to change; if unclear discussion resulted in unchanged answers; and if their safety or reputation felt at stake.

Results for the pre-meeting component of the survey were largely positive. Most panelists (79\%) agreed that the background information was sufficient and offered enough support; some (21\%) were uncertain and none disagreed. All panelists (100\%) agreed that the instructions were clear and easy to follow. All panelists (100\%) agreed that the expectations were well laid out. Most panelists (77\%) agreed that they were able to understand and complete all questions; some (23\%) were uncertain and none disagreed. Most panelists (93\%) agreed that the format was easy and unobtrusive.

Results for the in-person Delphi meeting component of the survey were more mixed but largely positive. All panelists (100\%) agreed that they felt their opinion mattered. Most panelists (93\%) disagreed that they felt disrespected; some (7\%) agreed. All panelists (100\%) agreed that their 
voice was heard and they could freely share their thoughts. Most panelists (71\%) disagreed that too much time was given to one panelist; some (21\%) were uncertain and some (7\%) agreed. All panelists $(100 \%)$ agreed that they were able to follow discussion. Most panelists $(79 \%)$ disagreed that they changed their answers due to intimidation; some (21\%) agreed and none were uncertain. Most panelists (93\%) disagreed that they kept their answers due to not understanding discussion; some (7\%) were uncertain and none agreed. All panelists (100\%) disagreed that they felt their safety and/or reputation were at stake.

Additional comments were invited with three questions: Was anything not addressed? Where is this research heading? And what would you change? Lastly, open space was provided for last thoughts.

The following are responses for items not addressed during the meeting:

"Maybe could have included more interventions"

"What were the successes in areas ASP is already implemented? i.e Acute Care so LTC can learn from this"

"I kind of wish there were things I could take away that I could implement right away"

The following are responses for where the research is heading:

"Development of guidelines for ASP in LTC”

"Coordinated approach to ASP in LTC"

"Believable quality improvement"

"Pragmatic research that evaluates a few specific outcomes (AMR rates, days of abx, classes of antimicrobials, mortality) in relation to interventions"

"Future research into the effectiveness of SCAT"

The following are responses to what panellists would change:

"Perhaps include another LTC physician"

"Some of the criteria were hard to interpret"

"Maybe increase anonymity to avoid "peer pressure" i.e. with prioritization. Some influence from facilitator that might have changed group's opinion"

The following are additional thoughts:

"I feel that many of the interventions were very ... on ... so it was hard to rate separately. i.e. most of the tools will be an enabler for guideline implementation 
Table 29. End of Day Survey Results

\begin{tabular}{|c|c|c|c|c|c|c|c|}
\hline Section & Question & $\% 1-2^{a}$ & Count $1-2$ & $\% 3^{\mathrm{b}}$ & Count 3 & $\% 4-5^{c}$ & Count $4-5$ \\
\hline \multirow{6}{*}{$\begin{array}{c}\text { Pre- } \\
\text { Meeting }\end{array}$} & $\begin{array}{l}\text { Background information } \\
\text { provided offered enough support }\end{array}$ & $0 \%$ & 0 & $21 \%$ & 3 & $79 \%$ & 11 \\
\hline & $\begin{array}{l}\text { Instructions were clear and easy } \\
\text { to follow }\end{array}$ & $0 \%$ & 0 & $0 \%$ & 0 & $100 \%$ & 14 \\
\hline & Expectations were well laid out & $0 \%$ & 0 & $0 \%$ & 0 & $100 \%$ & 14 \\
\hline & $\begin{array}{l}\text { Survey took appropriate time to } \\
\text { complete }\end{array}$ & $0 \%$ & 0 & $7 \%$ & 1 & $93 \%$ & 13 \\
\hline & $\begin{array}{l}\text { You were able to understand and } \\
\text { complete all questions }\end{array}$ & $0 \%$ & 0 & $23 \%$ & 3 & $77 \%$ & 10 \\
\hline & $\begin{array}{l}\text { Format was easy and } \\
\text { unobtrusive }\end{array}$ & $0 \%$ & 0 & $7 \%$ & 1 & $93 \%$ & 13 \\
\hline \multirow{8}{*}{$\begin{array}{l}\text { In-person } \\
\text { Delphi } \\
\text { Meeting }\end{array}$} & Opinion mattered & $0 \%$ & 0 & $0 \%$ & 0 & $100 \%$ & 14 \\
\hline & Felt disrespected & $93 \%$ & 13 & $0 \%$ & 0 & $7 \%$ & 1 \\
\hline & $\begin{array}{l}\text { Felt voice was heard and could } \\
\text { share thoughts }\end{array}$ & $0 \%$ & 0 & $0 \%$ & 0 & $100 \%$ & 14 \\
\hline & $\begin{array}{l}\text { Too much time was given to one } \\
\text { panellist }\end{array}$ & $71 \%$ & 10 & $21 \%$ & 3 & $7 \%$ & 1 \\
\hline & Able to follow discussion & $0 \%$ & 0 & $0 \%$ & 0 & $100 \%$ & 14 \\
\hline & $\begin{array}{l}\text { Changed answers because } \\
\text { intimidated }\end{array}$ & $79 \%$ & 11 & $0 \%$ & 0 & $21 \%$ & 3 \\
\hline & $\begin{array}{l}\text { Retained answers because could } \\
\text { not understand discussion }\end{array}$ & $93 \%$ & 13 & $7 \%$ & 1 & $0 \%$ & 0 \\
\hline & Safety and reputation at stake & $100 \%$ & 14 & $0 \%$ & 0 & $0 \%$ & 0 \\
\hline
\end{tabular}

\footnotetext{
${ }^{a}$ Percentage of scores for disagreement

${ }^{\mathrm{b}}$ Percentage of scores for somewhat agree/uncertainty

${ }^{\mathrm{c}}$ Percentage of scores for agreement
} 


\section{Chapter 4 Discussion}

\section{Key Objectives}

(1) To provide an overview of the survey results from the Modified Delphi Panel, including resourcing and prioritization, and an interpretation of such results in relation to the literature,

(2) To describe the results from the End of Day Survey, and

(3) To discuss strengths and limitations of the study, and future directions of the research. 


\section{Overview}

Many interventions for administering ASP interventions have been designed and trialed in acute hospital and ambulatory care; however, nursing homes have remained a relatively neglected focus for ASP research (Sloane et al., 2016; Daneman et al., 2011; Jump et al., 2012). As such research is limited, nursing home administrators attempting to implement ASP interventions often struggle with the selection of an intervention that will be feasible and effective (Malani et al., 2016). This thesis utilized the Modified Delphi Method to organize and evaluate a collection of ASP strategies (identified from previous literature and guidelines) for nursing home facilities. Thus, nursing homes are provided with a prioritized list of ASP interventions and a discussion of resources required for successful implementation.

\section{Summary of Results}

We organized a Modified Delphi panel to recruit individuals with expertise with ASPs and nursing home care for a daylong consensus panel with the intention of evaluating and prioritizing ASP interventions for nursing homes. Potential panelists were identified through contact with various health care organizations, such as FCO, OLTCA/OLTCC/OLTCP, and OARC, as well as identification from similar previous Modified Delphi panels (Morris et al., 2012; Dresser et al., 2017). Through this method, 36 candidate panel members were identified. This list was narrowed to 23 individuals after examination of the list of candidate panel members by members of the research team unaffiliated with the identification process. An official invitation of participation was sent to the 23 candidates; 16 accepted the invitation and attended the in-person meeting and completed Survey 2; 15 completed Survey 1 (Figure 5, Table 2).

This Modified Delphi process successfully evaluated ASP interventions for nursing home settings and narrowed interventions to those considered appropriate and necessary for nursing homes. Prior to Survey 1, 14 interventions were identified from the literature (Table 1), including sources such as IDSA Stewardship Guidelines and PHO's ASP Strategies (Barlam et al., 2016; PHO, 2016). Following Survey 2, 5 of the original 14 interventions plus 1 additional intervention were determined to be appropriate and relevant to nursing homes and were included for discussion of resourcing and prioritization: audit and feedback, clinical decision support systems, communication tools, guidelines for empiric prescribing, scheduled antibiotic reassessment 
(formerly de-escalation), and short course antibiotic therapy. The remaining ASP interventions were prioritized (Table 28): guidelines for empiric prescribing scored the highest priority (mean score of 1.3), audit and feedback and communication tools tied for second (mean score of 3.4), followed by short course antibiotic therapy (mean score of 3.9), scheduled antibiotic reassessment (mean score of 4.4), and clinical decision support systems (mean score of 4.9).

\section{Elimination of ASP Interventions}

Elimination of inappropriate and/or irrelevant ASP interventions for nursing homes was a critical component of this thesis. Two rounds of surveys were utilized to evaluate ASP interventions for nursing homes across five criteria: (1) scientific merit, (2) impact, (3) feasibility, (4) accountability, and (5) importance. Thus, following each discussion round was an opportunity to eliminate the intervention from discussing resourcing and prioritization.

\subsection{Elimination - Phase 1}

Following completion of Survey 1, which took place online prior to the in-person Delphi meeting, several ASP interventions were designated for elimination due to consensus for disagreement across all the evaluative criteria (scores between 7-9 accounting for less than 50\%): antibiotic cycling, formulary automatic substitution, formulary review, and IV to oral transition. Elimination of these interventions was deliberated with the panel during the in-person Delphi meeting; all were eliminated from discussion and Survey 2 excepting formulary automatic substitution. Disagreement regarding the application of present formulary automatic restriction for generic medication and the future extension to restricted antibiotics (explained further in the following section) warranted further discussion and inclusion for Survey 2.

\subsection{Elimination - Phase 2}

The remaining ASP interventions were discussed individually and evaluated through Survey 2 with the exception of multifaceted interventions. Multifaceted interventions were discussed briefly and, although receiving consensus for agreement for scientific merit and impact, the panel agreed that such an initiative would be infeasible for nursing homes. The panel thus decided that multifaceted interventions should be excluded from Survey 2 and eliminated from further discussion. This decision is in line with previous research that has indicated that multifaceted 
interventions are capable of significantly impacting inappropriate antibiotic prescriptions (Zabarsky et al., 2008; Arnold \& Straus, 2005); however, such interventions require extensive resources, which most nursing homes lack (Kennedy et al., 2014).

Upon completion and analysis of Survey 2 for the remaining 11 ASP interventions, discussion took place to determine which interventions ought to be considered appropriate and necessary for nursing homes and, thus, would be reserved for resourcing and prioritizing. Panelists were encouraged to express their opinions and feel free to disagree with the other panelists. Following discussion for each intervention, panelists were invited to complete Survey 2 for that specific intervention. Many interventions received lower scores across the evaluative criteria for Survey 2 than received for Survey 1 and, thus, were eliminated.

In Survey 1, antibiograms received scores for uncertainty for four out of the five criteria, whereas, in Survey 2, all five criteria received consensus for disagreement (Table 3 and 17). Thus, antibiograms were eliminated from further discussion. The decision to eliminate antibiograms fits well with previous literature. Nursing homes are generally lacking in access to standardized antibiograms (Kullar et al., 2017) and, although development of antibiograms is recommended by the IDSA Guidelines for ASP implementation (though a weak recommendation) (Barlam et al., 2016), such a practice would not be suited for nursing home settings and is preferential for acute hospital care. As referenced by a panelist, the most prominent infections in nursing homes include skin and soft-tissue infections (SSTIs), respiratory tract infections (RTIs) and, as mentioned earlier, UTIs (Nicolle at al., 1996). However, antibiograms would only beneficial for UTIs, as the other two infections do not include cultures (Furuno et al., 2014). Indeed, antibiograms are a valuable ASP initiative for acute care facilities; however, they are not yet practical for nursing homes.

Interventions for improving antibiotic use in health care facilities often rely on education. Educational interventions are a component of the CDC's 7 Core ASP Elements in LTC; however, nursing homes often lack educational resources (Kaki et al., 2011; Dyar et al., 2015). Nevertheless, in Survey 2, educational interventions received consensus for disagreement across all evaluative criteria, excepting feasibility (Table 21), and were eliminated from discussing resourcing and prioritization. Discussion surrounding educational interventions settled in agreement that such interventions are NBI - necessary but insufficient. Educational interventions 
have been studied extensively, and the general conclusion is that their effects are limited, especially when compared to other ASPs such as guideline implementation and audit and feedback (Freemantle et al., 1998; Arnold \& Straus, 2005). Education ultimately needs to be a component of any ASP intervention in nursing home settings; however, it alone is unlikely to spur lasting antibiotic prescribing changes (Schwartz et al., 2007).

Formulary automatic substitution received consensus for disagreement across all the evaluative criteria but was not eliminated during Phase 1 due to disagreement between panelists. Following discussion, panelists were invited to complete Survey 2 for formulary automatic substitution. Again, the intervention received consensus for disagreement across all criteria (Table 22). The ideology surrounding formulary automatic substitution is for pharmacists to automatically replace certain antibiotics with other, predetermined, antibiotics. The benefit for such an intervention is that restricted antibiotics are abandoned without prescribers actively replacing the antibiotic (PHO, 2016; Haas \& Bonczar, 1996). A form of formulary automatic substitution is already implemented in nursing home facilities - the automatic replacement of brand name medication with generic medication. Although such practices are not directly relevant to the automatic replacement of restricted antibiotics with similar unrestricted antibiotics, it strengthens the argument that such an intervention could possibly be effective in nursing homes. Unfortunately, evidence for the implementation of formulary automatic substitution in nursing homes is insufficient and, thus, cannot be a recommended intervention for such settings.

Much of the discussion for formulary restriction revolved around the potential for impact that such an intervention could have on antibiotic prescriptions. Implementing formulary restriction interventions in nursing home settings, however, would require provincial support, such as through Ontario Drug Benefit; an undertaking that would render implementation likely infeasible. Therefore, formulary restriction was eliminated from discussing resourcing and prioritization.

Following Survey 1, ASP interventions targeted for CDI received scores for uncertainty across all evaluative criteria. Discussion for the intervention was held and the panel proceeded to Survey 2, which resulted in consensus for disagreement across all evaluative criteria (Tables 16 and 26). Rates of CDI are significant in nursing homes, with $30 \%$ of residents treated with antibiotics ultimately acquiring CDI (Campbell et al., 2009; Thomas et al., 1990). Few panelists 
referred to their past experience with CDI in nursing homes, referring to its infrequency. It is possible that such anecdotal testimony is not representative of most nursing home facilities; however, further research is needed to confirm CDI prevalence in nursing homes.

\section{Resourcing}

\subsection{Communication Tools}

Among the initial 14 ASP interventions evaluated for implementation in nursing homes, 5 were determined to be appropriate and necessary, and were reserved for discussing resourcing and prioritization: (1) audit and feedback, (2) clinical decision support systems, (3) scheduled antibiotic reassessment, (4) guidelines for empiric prescribing, and (5) short course antibiotic therapy. A panel member suggested an additional intervention: communication tools.

"Structured communication forms can have a significant impact as an ASP, especially in nursing homes considering the contributing factor of miscommunication toward inappropriate prescribing",

Communication tools are interventions that target improved communication between providers, specifically, nurses, physicians, and pharmacists (McElligott et al., 2017; Gurwitz et al., 1990). As previously mentioned, a critical contributor to inappropriate antibiotic prescriptions in nursing homes is the lack of clear communication between nurses and physicians. Physicians are often off site and rely on nurses to accurately describe a resident's condition over the telephone. Ineffective communication often leads to misunderstanding and incorrect diagnoses of infections (Crnich et al., 2015; Katz \& Karuza, 2005). Structured communication tools provide nurses with a checklist of information to describe to physicians over the phone, such as a symptoms and vital signs (Haig KM et al., 2006).

An example of a structured communication tool that has been implemented in many health care settings is SBAR (Situation-Background-Assessment-Recommendation). SBAR consists of 7 components: (1) Situation, (2) Relevant Info, (3) Vital Signs, (4) Non-specific Signs and Symptoms (S/S), (5) Specific S/S, (6) Assessment, and (7) Review, Recommendation, and Notify (Haig et al., 2006; Sloane et al., 2016). Interventions targeting improved standardized assessment and interdisciplinary communication have been shown to reduce antibiotic use (Zimmerman et al., 2014; Katz et al., 2017). 
The panel evaluated communication tools interventions to be feasible for nursing homes (i.e. received consensus for agreement for feasibility) (Table 27). The feasibility and utility of implementing SBAR communication tools was studied in 137 nursing homes in Pennsylvania (Renz et al., 2013). Although the study did not report a conclusion regarding the feasibility of SBAR in nursing homes, the utility of SBAR was largely positive. Most nurses and physicians reported increased satisfaction with communication after implementation of SBAR. Nurses described feeling that SBAR provides structure for communication and "takes the guesswork out of the equation". McMaughan et al. (2016) reviewed the effectiveness of an SBAR decisionmaking aid in nursing homes for reducing antibiotic prescriptions for treating asymptomatic UTIs. The decision-making aid was associated with significantly reduced antibiotic prescriptions for suspected UTIs occurring without symptoms. Thus, although the effectiveness of some communication tool interventions is supported, the feasibility of such interventions is unclear. Further evidence is needed to conclude the feasibility of SBAR and other communication tools in nursing homes.

\subsection{Audit and Feedback}

Given a strong recommendation with moderate quality of evidence by the IDSA (Barlam et al., 2016), audit and feedback is an ASP intervention that has been reasonably investigated in health care, including acute hospital, ambulatory, and nursing home care (Brink et al., 2017; Arnold \& Straus, 2005). A resource that was discussed for audit and feedback was also referred for other interventions: better EMR ${ }^{1}$ implementation.

An EMR is a tool for collecting patient data in an electronic format, such as demographics and medical history, which can then be shared easily through a network system. EMRs are capable of significantly improving quality of care by reducing error, improving decision making and the sharing of health information (Gunter \& Terry, 2005; Kruse et al., 2017). A systematic review of EMRs in nursing homes found that implementation resulted in more positive (health, cost, patient satisfaction, etc.) than negative outcomes (decreased workflow and productivity) (Kruse et al., 2017). Although the need for improved use of EMR in many health care settings has been well established, implementation has been slower in nursing homes than that of acute hospital

\footnotetext{
${ }^{1}$ Electronic Medical Record. Or, alternatively, Electronic Health Record (EHR). There are slight differences between the two terms but this thesis uses the terms interchangeably.
} 
care and other health care settings (Broughton et al., 2013). A possible cause for the delayed implementation of EMRs into nursing homes is the cost of implementation and the lack of literature illustrating improved quality of life and decreased costs following implementation (Ajami \& Bagheri-Tadi, 2013). Other factors for the hesitant adoption of EMRs in nursing homes include perceptions of impact and the implementation process. Administrative and clinical user perceptions are major barriers toward implementing EMRs. Perceptions include misunderstanding the benefits of such tools, fear of change, belief of ineffectiveness, and concern for reduced time of physicians with residents. Furthermore, the process for implementing EMRs can be lengthy. A strategic plan must account for the size and needs of the facility, cost, and regulatory demands. An implementation team is an important factor for smooth implementation of an EMR (Kruse et al., 2015).

In addition to EMR, other resources required for implementation of audit and feedback interventions include a central data repository and a team for development of reports. Audit and feedback utilizes physician-prescribing data for peer comparison and improved antibiotic prescriptions. A central repository is needed in order to report on prescribing data. Furthermore, incorporating the data into a feedback document requires a team to organize the data.

Audit and feedback interventions are supported by the literature for being capable of reducing inappropriate antibiotic prescriptions (Arnold \& Straus, 2005). Audit and feedback was the only intervention to receive $100 \%$ consensus for agreement for scientific merit; however, it also scored for uncertainty for feasibility (Table 18). Thus, although likely capable of producing lasting change, such an intervention is resource-intensive and may not be feasible for all nursing home settings (Nicolle, 2014; McElligott et al., 2017).

\subsection{Clinical Decision Support Systems}

Clinical decision support system interventions are capable of effectively reducing inappropriate antibiotic prescriptions (Corsonello et al., 2015); the concern with the intervention is its feasibility. The panel agreed that clinical decision support systems are generally not feasible for nursing homes (received consensus for disagreement for feasibility) (Table 19). As aforementioned, EMRs provide health care staff access to extensive medical and demographic data. Such information is critical for implementing feasible decision support systems; however, 
until such an EMR is implemented, clinical decision support systems are likely infeasible for most nursing homes. Funding from MOHLTC is essential for future implementation.

\subsection{Scheduled Antibiotic Reassessment}

Implementing scheduled antibiotic reassessment interventions, where prescriptions of antibiotics are regularly reviewed and discontinued when necessary, would be a resource-intensive intervention (Pakyz AL et al., 2015). However, scheduled antibiotic reassessment received largely positive scores following Survey 2 . The only criterion that scheduled antibiotic reassessment did not receive consensus for agreement in Survey 2 was for feasibility, which scored for uncertainty (Table 20). Again, an EMR is a long-term solution, especially one that utilizes SBAR as such a tool would provide the necessary tracking of antibiotics and improve communication between staff. Additionally, as access to patient level data is central toward implementing scheduled antibiotic reassessment interventions, an EMR significantly increases the feasibility of such an intervention.

Concerns for implementation of scheduled antibiotic reassessment interventions in nursing homes include effective antibiotic tracking (i.e. continuity of care) and staffing issues (Kullar et al., 2017; Malani et al., 2016). Nursing home residents are frequently transferred between the facility and acute hospital care. During their time spent in acute care, many will receive antibiotic prescriptions that are not accounted for in the nursing home (Kullar et al., 2017). Thus, tracking all antibiotics prescribed to residents in nursing homes is a challenging task that requires interfacility, transferable patient-related medical data.

Beyond technical resources, human resources are an important requirement for implementing scheduled antibiotic reassessment interventions. Nursing homes are limited with staffing. In a review of nursing homes across Michigan, staffing constraints were a significant obstacle toward implementing ASP interventions (Malani et al., 2016). Staffing resources cited by panel members highlighted the need to include nursing home staff as ASP champions to ensure orders are reviewed effectively and consistently. A standardized procedure must be instituted, such as automatic nurse-led review of antibiotic prescriptions at day 3 of treatment. Funding from government agencies, such as MOHLTC, is a critical component for implementation of scheduled antibiotic reassessment interventions. 


\subsection{Guidelines for Empiric Prescribing}

Guideline development is an ongoing iterative process that has long been the method for improved quality of health care. Guidelines are constantly being developed for improved health care; however, guidelines are often targeted for acute hospital care. Furthermore, guidelines developed to target nursing homes are often not sufficiently adhered to and are often not implemented into policy (Sloane et al., 2016; Schuster et al., 1998; Davis \& Taylor-Vaisey, 1997). Guidelines are the backbone of many ASP initiatives but evidence supporting their effectiveness as a singular intervention is limited, which is the reason the panel designated the intervention as NBI (Katz et al., 2017; Nicolle, 2014). Antibiotic prescriptions for UTI treatment in nursing homes have been successfully improved using the Modified Loeb Criteria (Loeb et al., 2005). Guidelines are implemented to ensure that IV antibiotics are transitioned to oral antibiotics and that duration of therapy is not excessive (Naughton et al., 2001).

The panel agreed that implementing guidelines for empiric prescribing was feasible for nursing homes. In fact, it was the only intervention to receive consensus for agreement for all criteria in Survey 2 (Table 24). However, guidelines for empiric therapy vary and have generally only been reviewed for acute hospital care (File et al, 2004). Efforts to implement guidelines targeting empiric therapy in nursing homes have shown significant improvements for clinical outcomes (including mortality), adverse events, and costs; however, such guidelines are lacking in quality of evidence (Schuts et al., 2016).

Developing guidelines for improving empiric prescribing requires several resources. The panel suggested the following: a clinical practice guidelines (CPG) team ought to be recruited. The team would consist of a lead MD, nurse managers, an ID specialist, and nursing home residents and their advocates to ensure that the guidelines have a high degree of acceptability. Developing guidelines does not require starting from scratch; existing guidelines should be used as a foundation and expanded. Incorporating a centralized process to house guidelines, which would then be distributed and assessed for effectiveness at the local level, would benefit the feasibility of such interventions. The role of the CPG would also be to summarize current guidelines and prioritize those that are applicable to nursing home settings. It is unlikely that an initiative to improve guidelines for empiric prescribing will be national; provincial focus is the likely alternative. A representative from $\mathrm{HQO}$ explained that their organization is working toward 
synthesizing existing guidelines into simple statements. Once guidelines are organized, dissemination to nursing homes is necessary and would include webinars, electronic documents, and apps.

\subsection{Short Course Antibiotic Therapy}

Short course antibiotic therapy interventions have been relatively reviewed in nursing home settings; however, the intervention is lacking in overall scientific merit (i.e. received consensus for disagreement for scientific merit) (Table 25). A concern addressed by a panelist was prescribers' hesitancy to implement short course antibiotic therapy due to lack of evidence supporting its effectiveness. A small number of studies were identified from the literature reviewing short course antibiotic therapy, many of which examined implementation outside of nursing homes; those reviewing such interventions in nursing homes assessed for specific infections (UTIs). The evidence suggests that the intervention has high potential for reducing antibiotic consumption for specific infections. Short course therapy interventions for treatment of uncomplicated symptomatic UTIs for older adult women in nursing homes have seen success (Lutters \& Vogt-Ferrier, 2008; Vogel et al., 2004). Further evidence, especially RCTs, is required to confirm the applicability of short course antibiotic therapy for other indications and populations.

Resourcing requirements for implementing short course antibiotic therapy are similar to those suggested previously. Like scheduled antibiotic reassessment, short course antibiotic therapy interventions require effective monitoring of antibiotics. Nursing staff and physicians require access to prescription information, such as initiation date, class, and dose. Thus, an EMR could provide such requisite data and infection control nurses can be delegated to monitor prescriptions. Implementing such an intervention requires extensive legislative support. A panel member suggested that the Long Term Care Homes Act (LTCHA) must be updated to include ASPs. As of July 2010, all nursing homes in Ontario are governed by the LTCHA. The purpose of the LTCHA is to provide safe, consistent, and high-quality care for nursing home residents. Components of the Act include access to medicinal services, nutrition care, religious practices, etc. Access to medical services includes availability of medical services, individualized medical directives and orders, and attending physician or RN (LTCHA, 2007). ASPs are an element of 
many legislative organizations (CDC, PHO, IDSA, etc.). A component of the LTCHA must address the necessity of improving antibiotic use and implementation of ASP interventions.

\section{Prioritization}

A primary purpose of this thesis was to evaluate ASP interventions for implementation in nursing homes and prioritize the list of interventions to aid nursing homes in their selection of interventions. We successfully accomplished this task through the Modified Delphi Process. To our knowledge, no previous study has utilized the Modified Delphi Process to evaluate ASP interventions in nursing homes that could be applied nationally. The majority of research relating to ASPs has focused on acute hospital care and those that have addressed nursing homes have not provided a recommended implementation list. Extrapolating ASP research from hospital care to nursing homes is not a simple a task. Several factors limit the generalizability of translating evidence between hospital and nursing homes care, such as length of patient stay, age, cognitive impairment, and staffing (Sloane et al., 2016). Thus, the need for nursing home specific ASP research is critical.

In total, 15 interventions were evaluated and 6 were determined to be essential for nursing homes: (1) audit and feedback, (2) clinical decision support systems, (3) communication tools, (4) guidelines for empiric therapy, (5) scheduled antibiotic reassessment, and (6) short course antibiotic therapy. Using a ranking technique (using adhesive-backed posters set up around a room), panelists were able to rank the 6 interventions from most important to least. Raw scores for each intervention can be viewed in Table 28. Each intervention was given a score between 1 and 6. Mean scores were calculated for each.

The intervention given the highest priority was guidelines for empiric prescribing, which received a mean score of 1.3. This priority was expected. Guideline development represents a constantly evolving and fundamental aspect of policy and procedure in health care (Schuster et al., 1998; Grohl R \& Grimshaw J, 2003). Although, alone, guidelines are likely incapable of providing sustainable behaviour change, guideline development is foundational to the implementation of most ASP interventions (Charani et al., 2010). Local guideline development provides facility-specific guidance for antibiotic prescribing; however, local development is time 
consuming and infeasible for most nursing homes. Regional, provincial, and national guidelines are progressively more feasible and possibly more effective (Nicolle, 2014).

Empiric prescribing is almost always the pathway for initial treatment of suspected infections in nursing homes (Medina-Walpole \& McCormick, 1998). The panel determined that ASP interventions targeted at improving guidelines for empiric prescribing have enough scientific merit, have the most potential for impact, are generally feasible, are accountable, and are the priority for ASP interventions in nursing homes.

Two interventions tied for second priority, both receiving mean scores of 3.4: audit and feedback, and communication tools. Audit and feedback is a highly utilized intervention design in ASP research. A strong recommendation with moderate quality of evidence by the IDSA (Barlam et al., 2016), audit and feedback interventions have demonstrated a capacity to significantly improve antibiotic prescribing in acute hospital care and ambulatory settings (Arnold \& Straus, 2005). Audit and feedback in nursing homes, especially when incorporating peer comparison, have been shown to improve antibiotic prescriptions at the initiation, duration, and class level (Daneman et al., 2017). However, implementing audit and feedback into all nursing home settings is a resource intensive task that would greatly benefit from EMR and legislative support.

Communication is essential in all health care settings; however, in nursing homes, communication difficulties are abundant and transcend disciplines. Residents of nursing homes tend to be older with more cognitive impairments. Nursing staff often have trouble communicating with residents and may struggle to provide appropriate treatment (Dyar et al., 2015; Sloane et al., 2016; Genao \& Buhr, 2012). As discussed earlier, nursing homes frequently have off-site physicians who occasionally visit the facility. Nurses have to communicate residents' conditions over the phone in a manner that is concise and accurate to physicians. This places immense pressure on nurses. Nonstandardized telephone communication is a significant cause for inappropriate antibiotic prescriptions (Crnich et al., 2015). Additionally, poor communication between health care staff and pharmacy is another factor for inappropriate antibiotic prescriptions (Gurwitz et al., 1990). Thus, we highly recommend implementation of communication tool interventions that standardize communication, such as SBAR (Sloane et al., 2016). 
Short course antibiotic therapy interventions placed at third priority, receiving a mean score of 3.9. This priority is reasonable. The panel agreed that short course antibiotic therapy interventions have potential for impact and are useful for accountability purposes. The panel also agreed that such interventions are also feasible in nursing homes; short course antibiotic therapy was the only ASP to receive $100 \%$ consensus for agreement for feasibility. However, such an intervention is also considerably lacking in scientific merit. Most studies identified for short course antibiotic therapy in nursing homes were particular to uncomplicated symptomatic UTIs in older female adults, and many short course therapy studies are of poor quality of science (Lutters \& Vogt-Ferrier, 2008; Vogel et al., 2004). Thus, although short course antibiotic therapy is supported for specific instances in nursing homes, overall further evidence is needed (especially RCTs) to support its use across other indications (i.e. pneumonia, respiratory tract infection (RTIs)) and populations (i.e. older adult males).

Scheduled antibiotic reassessment interventions placed at fourth priority, receiving a mean score of 4.4. This ranking is slightly surprising, as the intervention received $100 \%$ consensus for agreement for impact and importance in Survey 2 (as well as high scores for accountability and scientific merit). "Antibiotic Timeouts", a component of scheduled antibiotic reassessment, are recommended in the literature. Such interventions have managed to significantly improve antibiotic use in hospitals (Crnich et al., 2015; Pakyz et al., 2015). Ultimately, the low ranking of scheduled antibiotic reassessment interventions in our priority list reflects the current feasibility of such interventions in nursing homes. It is reasonable to suspect that, once feasible, scheduled antibiotic reassessment interventions will be effective interventions for improving antibiotic prescriptions in nursing homes. However, until antibiotic tracking is improved, which requires better EMR implementation, it is unlikely that nursing homes will be able to implement scheduled antibiotic reassessment interventions.

Clinical decision support system interventions placed at fifth (and lowest) priority, receiving a mean score of 4.6. This placement is reasonable. Like scheduled antibiotic reassessment, clinical decision support systems are likely capable of producing lasting prescribing changes; the intervention received a strong consensus for agreement for impact (94\%) in Survey 2. However, the concern with clinical decision support system interventions is their feasibility. The panel arrived at consensus for disagreement for clinical decision support systems for feasibility. Although incorporating decision support tools would likely improve antibiotic prescriptions 
(Corsonello et al., 2015), implementation of such interventions into nursing homes is not currently feasible and, thus, is the lowest priority.

\section{Interpretation of Results}

\subsection{Panel Composition}

Our panel composition differed from the original Modified Delphi panel (RAND Method). The Modified Delphi method typically utilizes a panel composed entirely of experts in the field. Our panel, however, utilized non-experts as well as experts. The choice to include non-experts and diverge from the original Modified Delphi method was not simple. There was going to be a trade-off in the exclusion of potential experts, such as ASP and nursing home experts, for the purpose of including non-experts, such as residents, advocates, and councils. As aforementioned, residents and their advocates play an important role in the use of inappropriate antibiotic prescriptions. We believed it was essential to include residents and advocates in our Modified Delphi panel. Although ASP interventions require a high degree of acceptability for the prescribers and nursing staff, it is also critical that residents and advocates readily accept these interventions. The result of organizing a panel consisting of experts and non-experts ultimately led to the divergence of opinions noticeable in the comments following Survey 2.

\subsection{Divergence of Opinions}

Using the Modified Delphi Method, we managed to successfully evaluate ASP interventions for nursing homes. Although the process resulted in a prioritized list, the outcomes are not unquestionably clear. There remained some divergence of opinions regarding the final list of ASP interventions that are not reflective of the prioritized list. As previously mentioned, the constitution of the panel was heterogeneous and diverse. As such, differences of opinion between panelists are prominent in the comments following Survey 2, which may not be reflected in the final prioritized list.

Guidelines for empiric prescribing may have been the intervention to receive the highest priority; however, there remained some disagreement as to its potential for impact upon implementation. Most panel members view guidelines as foundational for effective ASP implementation, but the extent of its efficacy alone is unclear. One panelist suggested that for guidelines to be effective, 
they must be tailored to the local setting vs. regional setting vs. provincial setting vs. national setting. However, such effort would considerably limit the feasibility of the intervention.

Communication tools tied for second place priority, but some of the feedback following Survey 2 suggested it should have been placed lower. One particular comment referred to its similarity to structured communication forms, which that panel member concluded with "I'm not going to want to just go ahead with this in our home". This may be due to the lack of scientific merit supporting communication tool interventions in nursing homes. Communication tools surprisingly received such a high place priority with such opposing sentiment. Audit and feedback received consensus for agreement for scientific merit but was determined to have uncertain feasibility (the opposite for communication tools), which may explain the tied priority placement; however, this begs the question of equal weighting between criteria. Based on the final prioritized list of ASPs for nursing homes, it appears that feasibility should be considered the strongest criterion. Although an intervention may have the scientific evidence and potential impact to back up its implementation (i.e. multifaceted interventions), the feasibility of its implementation is the more important (policy implications-related) factor that determines its placement in the priority list.

As well, the feedback for short course antibiotic therapy was diverse. There was general agreement that the intervention had a high degree of feasibility. One panelist even suggested that this intervention "may be one of the easiest to implement". However, the divergence of opinion emerged in regards to the scientific merit. The results from the Survey 2 demonstrated that most panel members believed the intervention lacked scientific merit; however, one member commented that they were willing to set aside the lack of scientific evidence and extrapolate from other populations, such as acute hospital care. Again, the professional diversity of the panel is a likely reason for the divergence. Some panel members may be more experienced with the theoretical aspect of ASP research, whereas others may have more hands-on experience with implementation. It is possible that participants with experience in both acute care and nursing home settings may have felt more comfortable extrapolating evidence between health care settings. However, this contradicts much of the research highlighting the differences between nursing and acute care, particularly for ASP implementation (Sloane et al., 2016). Based on the placement of short course antibiotic therapy interventions in the priority list, it appears that the argument against its scientific merit trumped the desire to extrapolate evidence from other 
settings. Indeed, the final prioritized list leads us to conclude that feasibility is the determining factor for ASP implementation in nursing homes; however, what this means for policy implications may be different.

\subsection{Policy Implications}

Feasibility is arguably the most important factor to consider for intervention implementation in nursing homes (Kennedy et al., 2014). The reason that clinical decision support systems, an ASP that was determined to have a high potential for impact, received such a low priority ranking is explained by its feasibility. Nursing homes do not have the resources to implement clinical decision support system interventions. Furthermore, multifaceted interventions, which received high scores for both scientific merit and impact, was eliminated following Survey 1 because of feasibility concerns. Indeed, it seems that feasibility issues are capable of eliminating the most evidence-informed approach, which in this case is multifaceted interventions. As mentioned earlier, behaviour change is a challenging task, especially for prescribers with historical tendencies to consistently prescribe inappropriate rates, durations, and doses of antibiotics (Michie et al., 2011; Daneman et al., 2017). Arnold \& Straus' (2005) systematically reviewed ASP interventions in ambulatory care. Studies included were RCTs and quasi-RCTs, controlled before/after studies and interrupted time series studies. Multifaceted interventions were found to be more successful for reducing inappropriate antibiotic prescriptions than individual interventions. However, nursing homes generally do not have the resources available to implement multifaceted interventions. The question remains: assuming nursing homes have the resources to implement individual interventions, will there be noticeable changes in inappropriate antibiotic use after implementation?

Guidelines for empiric prescribing received the highest priority score and received consensus for agreement for potential for impact (as well for the remaining criteria). However, comments following Survey 2 questioned its capacity for prescribing changes. For instance, the intervention was ranked highest priority; yet, one comment received was that it was NBI - necessary but insufficient. The intervention may have been placed as highest priority as it is fundamental to all ASPs in nursing homes; however, if nursing homes cannot bundle the intervention with other components, there likely will not be significant prescribing changes. Thus, the only solution to 
this challenge (i.e. the need for multifaceted interventions while lacking resources to implement multifaceted interventions) may be to increase resources devoted to nursing home care.

This study managed to prioritize ASP interventions for nursing homes, but this is only beneficial if the facilities can incorporate other interventions to improve the potential for impact. In order to do so, nursing homes need better access to resources, particularly staffing, funding, and EMR implementation.

\section{Strengths and Limitations}

\subsection{Strengths}

Many studies have reviewed the effectiveness of several ASP interventions in acute hospital and ambulatory care (Davey et al., 2017; Arnold \& Straus, 2005). Many studies have examined the use of antibiotics in nursing homes, have argued for the need for ASPs, and have tested the effectiveness of individual and multifaceted interventions in nursing homes (Strausbaugh \& Joseph, 2000; Hughes, 2011; Jump et al., 2012). However, to our knowledge, no previous study has thoroughly examined and contrasted such a robust number of ASP interventions as we have, and prioritized the interventions for aiding nursing homes in the selection and implementation of ASP interventions to improve antibiotic prescriptions.

The use of the Modified Delphi Method has its strengths. Opinions for initiatives to improve health care are often diverse, especially for areas where the literature is inconclusive, such as ASP research in nursing homes. The Delphi Method is unique as it provides a tool for converging the various outlooks toward agreement, while also being highly feasible (Lyons, 1981). Utilizing the Modified Delphi Method, which included the in-person Delphi meeting, provided panelists with an environment that welcomed open-ended discussion.

The interdisciplinary representativeness of the panel was an asset to our study. The diversity of the panel provided expert and non-expert opinion, and shared personal experience. Renowned ASP researchers were present for the in-person Delphi meeting to discuss their research findings, describe the complexity of ASP implementation, and relate ASP literature from other health care settings to nursing homes. Furthermore, to provide more inclusive perspectives, residents of nursing homes and family council representatives were invited to - and participated in - the 
meeting. Thus, while ASP experts could speak to the effectiveness of ASP interventions from the literature, residents and family councils could provide perspective from their own experiences within nursing homes. Nurses, physicians, and pharmacists were an obvious inclusion. As nursing home staff are the usual target for ASP interventions, receiving their feedback and understanding their outlook for each intervention was an invaluable component of the panel. Moreover, implementing ASP interventions requires administrative and legislative support. Our panel included nursing home administration and representatives from government health care bodies, such as PHO and HQO.

Panelists were provided an opportunity to reflect on their panel experience with the End of Day Survey and the clear majority of responses were positive. All panelists agreed that instructions were clear and easy to follow and that expectations were well laid out. Moreover, all panelists agreed that their opinion mattered, their voice was heard, they were able to comprehend the discussion, and that they felt safe. Almost all panelists (93\%) believed the survey took an appropriate amount of time to complete, the format was easy to understand and follow, they never felt disrespected, and they did not retain answers due to not understanding the discussion. Most panelists (71-79\%) believed the background information was sufficient, they could understand and complete all questions, and not too much time was given to one panelist, and they were not intimidated to change their answers.

\subsection{Limitations}

Our study has limitations. The first limitation relates to the representation of the panel. Selection bias may have occurred. Although an interdisciplinary panel provides more diverse opinion, it is arguable that the discussion would have benefitted from more nursing home and ASP experts (rather than residents and their advocates). Our panel was limited (unintentionally) to one nursing home physician. Though two hospital physicians were present, our panel would have benefitted from additional nursing home physicians.

The intention of our study was to utilize a professionally and geographically diverse expert and non-expert panel to evaluate ASP interventions for nursing homes. Although the panel was professionally diverse, geographically it was not. The lack of geographical representation is a limitation to our study. The vast majority of the panel participants were located within the GTA (as used in previous, similar Modified Delphi Panels), three participants were recruited from 
within Ontario (outside of the GTA) and two were recruited from outside of Ontario (within Canada). Much of the reason for the lack of geographical diversity stems from feasibility constraints and a lack of identified potential participants from outside Ontario.

One of the main limitations this study faced was ensuring all panelists could understand the discussion and provide free input and opinions. It is likely that individual panel members steered the conversation more than others. It was inevitable that, due to prior knowledge and expertise, some strong personalities would overshadow others during the discussion. Panelists with ASP expertise were likely to be biased toward the interventions they have reviewed. We attempted to organize a comprehensive background package that was given to all panel participants to level the knowledge gap. However, following the End of Day Survey, some panelists (3) responded with uncertainty when asked if the background information was sufficient and whether they could understand and complete all questions. When asked if too much time was given to one panelist, 3 panelists responded with uncertainty and 1 agreed. When asked if panelists ever felt disrespected, the vast majority responded disagreement; however, there was one panelist who responded with agreement. If a panelist truly felt disrespected, it is essential that future Modified Delphi panels work to ensure it does not recur. For instance, by asking for feedback from panelists earlier in the process to allow for swift attention and correction. Additionally, more effort can be made to approach soft-spoken panelists to ensure that they have an equal opportunity to share their opinions.

Another limitation of this study was potential influence from the facilitator. The role of the facilitator is to encourage and help lead open discussion. It is possible that the facilitator may have implicitly encouraged certain opinion over others. Indeed, one panelist did refer to a potential bias being "some influence from facilitator that might have changed group's opinion".

Another limitation of this study is a potential bias during the prioritization component. The initial strategy was for panelists to rank interventions by filling out 6 small adhesive-backed paper cards (labeling each from 1-6) and placing them on the wall at designated areas. However, individual adhesive-backed cards were not available and the arrangement was changed to place 6 large posters on the walls, representing the 6 ASP interventions, and have the panelists approach each and write a number (from 1-6) directly on the poster. Although this method successfully resulted in a prioritized list of ASP interventions, it is possible that panelists were influenced by 
others to place similar rankings. Indeed, one panelist referred to this in the "additional comments" component of the End of Day Survey, and although the majority (79\%) of panelists disagreed that answers were changed due to intimidation, some (21\%) agreed. Overall, the results from the End of Day Survey suggest that social pressures did not meaningfully influence the outcomes of this study.

Another limitation of this study is the reliability and validity of the results. The Delphi Method has generally been criticized for arriving at conclusions that are dependent upon the composition of the panel. This concern is greatest when scientific evidence for the topic is lacking. Studies examining the reliability and validity of the Delphi Method have argued for the need of multidisciplinary representativeness to prevent groupthink (Leape et al, 1992; Coulter et al., 1995). Additionally, the methodology for prioritizing the ASP interventions has its limitations. Due to the low sample size, it is difficult to determine the significance of the mean score differences. Guidelines for empiric prescribing emerged with a mean score strikingly higher than other interventions, with a mean score of 1.3. The means scores for the remaining interventions were much closer in range. The second place interventions were tied with mean scores of 3.4, but the third place intervention received a score of 3.9, and the remaining were 4.4 and 4.6. Thus, while these mean scores provided us with a means of prioritizing the list of ASP interventions for nursing homes, the question as to whether these scores are representative of how such interventions ought to be prioritized is unclear.

\section{Conclusions}

This study sheds light on an overlooked area of research: antibiotic use in nursing homes. Antibiotic use in nursing homes is a complicated issue but, overall, antibiotic prescriptions remain largely inappropriate. The causes for such inappropriateness are variable. Several factors influence the increasing (and often inappropriate) use of antibiotics in nursing homes, such as residents' cognition and comorbidities, and communication between nurses and physicians. Internal efforts to improve antibiotic use are few and largely unsuccessful. Standardized, evidence-based approaches to antibiotic prescribing are needed to improve antibiotic utilization in nursing homes. 
Antimicrobial stewardship programs refer to coordinated health care initiatives and interventions that seek to optimize antimicrobial (i.e. antibiotic) use, whilst minimizing the occurrence of adverse drug events, costs of therapy, and preventing the spread of antimicrobial resistance. ASP interventions have been investigated and successfully implemented into some health care settings, such as acute hospital and ambulatory care. However, ASP implementation in nursing homes remains mostly unexplored, even though such facilities are in need for these programs. Furthermore, existing evidence for ASP implementation in nursing homes is inconsistent and may not be a suitable determining factor for ASP selection in such facilities. There are many strategies for implementing ASPs and nursing homes looking to organize ASP interventions often struggle with intervention selection and implementation of these programs.

This study utilized the Modified Delphi Process, a tool for arriving at consensus, to evaluate and prioritize ASP interventions for nursing homes and describe the resourcing required for implementation. An interdisciplinary and nationally representative panel was invited to participate in two rounds of surveys and an in-person Delphi meeting. The interdisciplinary and national representation of the panel ensures that the selected interventions are acceptable across all designations within the nursing home setting and across the country. The Delphi meeting took place in Toronto, ON, at the Sinai Health Care System, on March 20, 2018 at 9:00am.

A total of 15 interventions for administering ASPs were identified and evaluated; 6 were determined to be appropriate and necessary for nursing home settings and were prioritized in the following order: (1) guidelines for empiric prescribing, (2) audit and feedback and communication tools (tied), (3) short course antibiotic therapy, (4) scheduled antibiotic reassessment, and (5) clinical decision support systems. Resourcing requirements for implementing each intervention is highly variable, yet there are commonalities shared between, such as improving EMR implementation and acquiring funding and other support from government agencies, such as MOHLTC. Thus, through this study, nursing homes are provided with a tool for selecting an ASP intervention(s) that suits the institution's feasibility and a description of the resources required to implement such programs.

\section{Future Directions}

The future of ASP research in nursing homes is rich with potential. The current state of ASP research is mostly focused on acute hospital care settings. Most nursing home administrators are 
enthusiastic about implementing ASP interventions but few have been initiated due to a multitude of factors such as feasibility, inconsistency in the guidelines, and a general lack of knowledge relating to ASP selection and implementation. Soon, however, ASP interventions will be implemented in all nursing homes, antibiotic prescriptions will be more optimized, overall consumption will likely decrease, and the spread of AMR will be reduced.

\subsection{Improved Evidence Base}

Improving ASP implementation requires improving the scientific evidence for such interventions. Many ASP interventions are unsuccessfully implemented in nursing homes using evidence extrapolated from other health care settings. This study assessed ASP interventions in nursing homes using scientific merit as an evaluative criterion; however, not all ASP interventions have been thoroughly investigated in nursing home facilities. Such facilities have characteristics that distinguish them from other health care settings. Extrapolating ASP research evidence from other health care settings is problematic and is often unsuccessful for sustainably improving antibiotic use. Altering prescribing behaviours is a difficult task; however, clinicians are more likely to change their behaviour when interventions are well supported in the literature. Greater efforts are needed to assess ASP implementation in nursing homes to gain better insight into the barriers and facilitators of each intervention for improved comparisons. Particularly, high priority research, such as RCTs for short course interventions, is needed to support the implementation of potentially beneficial interventions (Barlam et al., 2016).

\subsection{Implications}

Future knowledge translation work will continue beyond this thesis to engage stakeholder groups for policy and procedure changes in nursing homes to accommodate ASP implementation. ASP implementation needs to be included within nursing home policy and procedure. As discussed earlier, the Long Term Care Homes Act (LTCHA) governs all nursing homes across Ontario and ensures residents receive safe, consistent, and high-quality care. ASPs are yet to be included in the LTCHA but should be if there is to be better ASP implementation across all nursing homes.

Meetings with stakeholders will focus on developing improved guidelines for administering antibiotic therapy in nursing homes, particularly targeting empiric therapy. Additionally, guidelines can be developed to improve implementation of short course therapy durations. 
Collaboration with government bodies, such as MOHLTC and HQO, will be beneficial for improving ASP funding and providing a coordinated approach to implement ASP interventions in nursing homes.

An important component in the effort to implement ASP interventions in nursing homes is improving the feasibility of such programs. One of the main criteria for an intervention to be effective for driving clinical practice change in nursing homes is the feasibility of its implementation (Loeb et al., 2002). Multifaceted interventions received moderately high scores for Survey 1, particularly for scientific merit and impact; however, the intervention was eliminated prior to Survey 2 due to feasibility restraints. Many other interventions may also have a high degree of potential for impact but, ultimately, nursing homes need to be able to implement the programs. Nursing homes do not have the same access to resources as other health care settings, such as acute hospital and ambulatory care, and, thus, are limited with which ASP interventions can be implemented (Kennedy et al., 2014).

As described in this thesis, a tool that will improve the feasibility of ASP interventions is the implementation of EMRs. EMRs provide access to extensive patient-related data. Such data access is essential for many ASP interventions, such as audit and feedback, clinical decision support systems, and scheduled antibiotic reassessment. However, EMR implementation has not been adequately evaluated in nursing homes; it is difficult to determine what outcomes will take place, such as nursing staff work time, after implementation. EMRs should provide nursing staff with alerts and notifications for changes in resident conditions, thus, improving nursing staff collaboration (Bowers \& Benford, 1991), but one study found that implementation only operated as a data repository. Communication practices did evolve over time from oral to electronic, implying that EMR implementation requires a lengthy time for adaptation (Munyisia et al., 2014). As EMRs continue to be implemented into more nursing homes, so too, many more ASP interventions will likely be more feasible.

\subsection{Novel Interventions}

Lastly, the development of novel, high-quality interventions is the next step in the effort to improve antibiotic utilization in nursing homes. Many ASP interventions have been developed and are moderately supported in the literature; 15 ASP interventions were identified for this study. However, the effectiveness of existing interventions is limited and inconsistent. The 
antibiotic prescribing process is complex. With every step in the process, there is potential for miscommunication and pressure that prompts the prescription of an inappropriate antibiotic. To meaningfully improve antibiotic use in nursing homes, novel and high-quality ASP interventions must be developed that target all areas of the prescribing process.

As discussed previously, to improve inappropriate antibiotic prescriptions in nursing homes likely requires implementation of some form of multifaceted intervention. However, it was also mentioned that nursing homes do not have the resources to implement multifaceted interventions. Thus, further research is needed to explore potential intervention bundles, where implementation of such a bundle is feasible for nursing homes and likely to result in sustainable prescribing changes. In this way, the bundle itself and the sequencing of the separate interventions would be informed by implementation and scaling considerations.

Pragmatic research is needed that evaluates specific outcomes of ASP interventions in nursing homes. A common outcome measure of ASP interventions is days of antibiotic therapy. Novel ASP interventions must be designed to target and improve mortality due to inappropriate antibiotic consumption, with respect to particular classes of antibiotics. An exciting future prospect is the design of ASP interventions that directly measure and decrease AMR rates. 


\section{Bibliography}

1. Gualerzi CO, Brandi L, Fabbretti A, Pon CL, editors. Antibiotics: Targets, mechanisms and resistance. John Wiley \& Sons; 2013 Sep 5.

2. Burki T. Antibiotic development pipeline slows to a trickle. The Lancet Infectious Diseases. 2017 Nov 1;17(11):1128-9.

3. Braine T. Race against time to develop new antibiotics. Bull World Health Organ. 2011;89(2):88-9. IDSA, 2004

4. Hughes JM. Preserving the lifesaving power of antimicrobial agents. JAMA. 2011;305(10):1027-8.

5. Wenzel RP. The antibiotic pipeline — challenges, costs, and values. New England Journal of Medicine. 2004 Aug 5;351(6):523-6.

6. Kohanski MA, Dwyer DJ, Collins JJ. How antibiotics kill bacteria: from targets to networks. Nature Reviews Microbiology. 2010 Jun;8(6):423.

7. Morrill HJ, Caffrey AR, Jump RL, Dosa D, LaPlante KL. Antimicrobial Stewardship in Long-Term Care Facilities: A Call to Action. J Am Med Dir Assoc. 2016;17(2):183.e1-16.

8. Roberts KD, Azad MA, Wang J, Horne AS, Thompson PE, Nation RL, et al. Antimicrobial Activity and Toxicity of the Major Lipopeptide Components of Polymyxin B and Colistin: Last-line Antibiotics against Multidrug-Resistant Gram-negative Bacteria. ACS Infect Dis. 2015;1(11):568-75.

9. Drinka P, Niederman MS, El-Solh AA, Crnich CJ. Assessment of risk factors for multidrug resistant organisms to guide empiric antibiotic selection in long term care: a dilemma. Journal of the American Medical Directors Association. 2011 Jun 1;12(5):321-5.

10. Spellberg B, Guidos R, Gilbert D, Bradley J, Boucher HW, Scheld WM, et al. The epidemic of antibiotic-resistant infections: a call to action for the medical community from the Infectious Diseases Society of America. Clin Infect Dis. 2008;46(2):155-64.

11. Centers for Disease Control and Prevention (CDC). Antibiotic resistance threats in the United States, 2013. Atlanta: CDC; 2013. 
12. Bonomo RA. Multiple antibiotic-resistant bacteria in long-term-care facilities: an emerging problem in the practice of infectious diseases. Clinical Infectious Diseases. 2000 Dec $1 ; 31(6): 1414-22$.

13. Nicolle LE. Urinary tract infection in long-term-care facility residents. Clin Infect Dis. 2000;31(3):757-61.

14. Turnbaugh PJ, Ley RE, Hamady M, Fraser-Liggett CM, Knight R, Gordon JI. The human microbiome project. Nature. 2007 Oct 17;449(7164):804.

15. Barbara G, Feinle-Bisset C, Ghoshal UC, Santos J, Vanner SJ, Vergnolle N, Zoetendal EG, Quigley EM. The intestinal microenvironment and functional gastrointestinal disorders. Gastroenterology. 2016 May 1;150(6):1305-18.

16. Cai T, Mazzoli S, Mondaini N, Meacci F, Nesi G, D'elia C, Malossini G, Boddi V, Bartoletti R. The role of asymptomatic bacteriuria in young women with recurrent urinary tract infections: to treat or not to treat?. Clinical infectious diseases. 2012 Jun 7;55(6):7717.

17. Iizumi T, Battaglia T, Ruiz V, Perez GI. Gut microbiome and antibiotics. Archives of medical research. 2017 Dec 6.

18. Drinka PJ, Stemper ME, Gauerke CD, Miller JE, Reed KD. Screening for methicillinresistant Staphylococcus aureus in a nursing home. Infection Control \& Hospital Epidemiology. 2004 Feb;25(2):95-6.

19. O'Neill J. Antimicrobial resistance: tackling a crisis for the health and wealth of nations. Rev. Antimicrob. Resist. 2014 Dec 11;20:1-6.

20. Adeyi OO, Baris JE, Olga B, Irwin A, Berthe FCJ; Le Gall FG, Marquez PV. Nikolic IA, Plante, CA, Schneidman MS, Donald E, Thiebaud A. 2017. Drug-resistant infections : a threat to our economic future (Vol. 2) : final report (English). Washington, D.C. : World Bank Group. http://documents.worldbank.org/curated/en/323311493396993758/finalreport

21. Centers for Disease Control and Prevention (CDC). Antibiotic resistance threats in the United States, Atlanta: U.S. Department of Health and Human Services, Centers for Disease Control and Prevention; 2013. [Internet] Available from: https://www.cdc.gov/drugresistance/threat-report-2013/pdf/ar-threats-2013-508.pdf\#page53 
22. European Centre for Disease Prevention and Control (ECDC). Summary of the latest data on antimicrobial resistance in EU. Stockholm: European Centre for Disease Prevention and Control; 2016. [Internet] Available from:

https://ecdc.europa.eu/sites/portal/files/documents/antibiotics-EARS-Net-summary2016_0.pdf

23. Roberts RR, Hota B, Ahmad I, Scott RD, Foster SD, Abbasi F, Schabowski S, Kampe LM, Ciavarella GG, Supino M, Naples J. Hospital and societal costs of antimicrobial-resistant infections in a Chicago teaching hospital: implications for antibiotic stewardship. Clinical infectious diseases. 2009 Oct 15;49(8):1175-84.

24. Costelloe C, Metcalfe C, Lovering A, Mant D, Hay AD. Effect of antibiotic prescribing in primary care on antimicrobial resistance in individual patients: systematic review and meta-analysis. BMJ. 2010;340:c2096.McCaig 1995

25. McCaig LF, Hughes JM. Trends in antimicrobial drug prescribing among office-based physicians in the United States. Jama. 1995 Jan 18;273(3):214-9.

26. Charani E, Cooke J, Holmes A. Antibiotic stewardship programmes--what's missing? J Antimicrob Chemother. 2010;65(11):2275-7.Harbath 2001

27. Harbarth S, Harris AD, Carmeli Y, Samore MH. Parallel analysis of individual and aggregated data on antibiotic exposure and resistance in gram-negative bacilli. Clinical Infectious Diseases. 2001 Nov 1;33(9):1462-8.

28. Donnan PT, Wei L, Steinke DT, Phillips G, Clarke R, Noone A, et al. Presence of bacteriuria caused by trimethoprim resistant bacteria in patients prescribed antibiotics: multilevel model with practice and individual patient data. BMJ. 2004;328(7451):1297.

29. Canadian institution for Health Information. Health Spending - Nursing Homes. 2014. Available from: https://secure.cihi.ca/free_products/infosheet_Residential_LTC_Financial_EN.pdf

30. Ribbe MW, Ljunggren G, Steel K, Topinková E, Hawes C, Ikegami N, et al. Nursing homes in 10 nations: a comparison between countries and settings. Age Ageing. 1997;26 Suppl 2:3-12.

31. National Center for Health Statistics (US). The National Nursing Home Survey: 2004 overview. Dept. of Health and Human Services Centers for Disease Contr; 2009.

32. Mody L, Langa KM, Saint S, Bradley SF. Preventing infections in nursing homes: a survey of infection control practices in southeast Michigan. Am J Infect Control. 2005;33(8):489-92. 
33. Long-Term Care Utilization Report, February 2018, Ontario Ministry of Health and LongTerm Care; Ontario Long Term Care Association, internal database, 2018.

34. Smith PW, Bennett G, Bradley S, Drinka P, Lautenbach E, Marx J, Mody L, Nicolle L, Stevenson K. SHEA/APIC guideline: infection prevention and control in the long-term care facility. Infection Control \& Hospital Epidemiology. 2008 Sep;29(9):785-814.

35. Mody L. Infection control issues in older adults. Clinics in geriatric medicine. 2007 Aug $1 ; 23(3): 499-514$.

36. Trick WE, Weinstein RA, DeMarais PL, Kuehnert MJ, Tomaska W, Nathan C, Rice TW, McAllister SK, Carson LA, Jarvis WR. Colonization of skilled-care facility residents with antimicrobial-resistant pathogens. Journal of the American Geriatrics Society. 2001 Mar; 49(3):270-6.

37. Corsonello A, Abbatecola AM, Fusco S, Luciani F, Marino A, Catalano S, et al. The impact of drug interactions and polypharmacy on antimicrobial therapy in the elderly. Clin Microbiol Infect. 2015;21(1):20-6.High 2009

38. High KP, Bradley SF, Gravenstein S, Mehr DR, Quagliarello VJ, Richards C, et al. Clinical practice guideline for the evaluation of fever and infection in older adult residents of long-term care facilities: 2008 update by the Infectious Diseases Society of America. Clin Infect Dis. 2009;48(2):149-71.

39. Nicolle LE. Antimicrobial stewardship in long term care facilities: what is effective? Antimicrob Resist Infect Control. 2014;3(1):6.Friedrich 2018

40. Friedrich MJ. Antibiotic Consumption Increasing Globally. Jama. 2018 May 15;319(19):1973-.

41. Klein EY, Van Boeckel TP, Martinez EM, Pant S, Gandra S, Levin SA, Goossens H, Laxminarayan R. Global increase and geographic convergence in antibiotic consumption between 2000 and 2015. Proceedings of the National Academy of Sciences. 2018 Mar 21:201717295.

42. Daneman N, Gruneir A, Newman A, Fischer HD, Bronskill SE, Rochon PA, et al. Antibiotic use in long-term care facilities. J Antimicrob Chemother. 2011;66(12):2856-63.

43. Nicolle LE, Bradley S, Colgan R, Rice JC, Schaeffer A, Hooton TM. Infectious Diseases Society of America guidelines for the diagnosis and treatment of asymptomatic bacteriuria in adults. Clinical Infectious Diseases. 2005 Mar 1:643-54. 
44. Peron EP, Hirsch AA, Jury LA, Jump RL, Donskey CJ. Another Setting for Stewardship: High Rate of Unnecessary Antimicrobial Use in a Veterans Affairs Long-Term Care Facility. Journal of the American Geriatrics Society. 2013 Feb;61(2):289-90.

45. Sergi G, De Rui M, Sarti S, Manzato E. Polypharmacy in the elderly. Drugs \& aging. 2011 Jul 1;28(7):509-18.

46. Medeiros EF, Moraes CF, Karnikowski M, Nóbrega OT, Karnikowski MG. An interdisciplinary intervention as a strategy for Rational Use of Drugs by the elderly. Ciencia \& saude coletiva. 2011 Jul;16(7):3139-49.

47. Ramage-Morin PL. Medication use among senior Canadians. Health Rep. 2009;20(1):37-44.

48. Fulton MM, Riley Allen E. Polypharmacy in the elderly: a literature review. Journal of the American Academy of Nurse Practitioners. 2005 Apr;17(4):123-32.

49. NCHS. Nursing and Related Care Homes as Reported From the 1986 Inventory of LongTerm Care Places. Advancedata . No. 147. DHHS Publ. No. (PHS) 88-1250. Public Health Service. Hyattsville, Md.: National Center For Health Statistics, January 22, 1988a.

50. van Buul LW, van der Steen JT, Veenhuizen RB, Achterberg WP, Schellevis FG, Essink RT, et al. Antibiotic use and resistance in long term care facilities. J Am Med Dir Assoc. 2012;13(6):568.e1-13.

51. Field TS, Gurwitz JH, Avorn J, McCormick D, Jain S, Eckler M, et al. Risk factors for adverse drug events among nursing home residents. Arch Intern Med. 2001;161(13):162934.

52. Nolan L, O'Malley K. Prescribing for the elderly part I: sensitivity of the elderly to adverse drug reactions. Journal of the American Geriatrics Society. 1988 Feb;36(2):142-9.

53. Gurwitz JH, Field TS, Avorn J, McCormick D, Jain S, Eckler M, et al. Incidence and preventability of adverse drug events in nursing homes. Am J Med. 2000;109(2):87-94.

54. Gurwitz JH, Soumerai SB, Avorn J. Improving medication prescribing and utilization in the nursing home. J Am Geriatr Soc. 1990;38(5):542-52.Ilić 2015

55. Ilić D, Bukumirić Z, Janković S. Impact of educational intervention on prescribing inappropriate medication to elderly nursing homes residents. Srpski arhiv za celokupno lekarstvo. 2015;143(3-4):174-9. 
56. Katz PR, Beam TR, Brand F, Boyce K. Antibiotic use in the nursing home: Physician practice patterns. Archives of internal medicine. 1990 Jul 1;150(7):1465-8.

57. Loeb M. Antibiotic use in long-term-care facilities: many unanswered questions. Infection Control \& Hospital Epidemiology. 2000 Oct;21(10):680-3.

58. Forbes D, Withers G. Prophylactic therapy in cyclic vomiting syndrome. J Pediatr Gastroenterol Nutr. 1995;21 Suppl 1:S57-9.Pickard 2018.

59. Pickard R, Chadwick T, Oluboyede Y, Brennand C, von Wilamowitz-Moellendorff A, McClurg D, Wilkinson J, Ternent L, Fisher H, Walton K, McColl E. Continuous low-dose antibiotic prophylaxis to prevent urinary tract infection in adults who perform clean intermittent self-catheterisation: the AnTIC RCT. Health Technology Assessment. 2018.

60. Daneman N, Bronskill SE, Gruneir A, Newman AM, Fischer HD, Rochon PA, et al. Variability in Antibiotic Use Across Nursing Homes and the Risk of Antibiotic-Related Adverse Outcomes for Individual Residents. JAMA Intern Med. 2015;175(8):1331-9.

61. Benoit SR, Nsa W, Richards CL, Bratzler DW, Shefer AM, Steele LM, et al. Factors associated with antimicrobial use in nursing homes: a multilevel model. J Am Geriatr Soc. 2008;56(11):2039-44.

62. Mylotte JM. Antimicrobial prescribing in long-term care facilities: prospective evaluation of potential antimicrobial use and cost indicators. American journal of infection control. 1999 Feb 1;27(1):10-9.

63. Carter RR, Montpetite MM, Jump RLP. Mixed-Methods Pilot Study to Assess Perceptions of Antimicrobial Stewardship in Nursing Homes. J Am Geriatr Soc. 2017;65(5):1073-8.

64. Dyar OJ, Pagani L, Pulcini C. Strategies and challenges of antimicrobial stewardship in long-term care facilities. Clin Microbiol Infect. 2015;21(1):10-9.

65. Crnich CJ, Jump R, Trautner B, Sloane PD, Mody L. Optimizing Antibiotic Stewardship in Nursing Homes: A Narrative Review and Recommendations for Improvement. Drugs Aging. 2015;32(9):699-716.

66. Lam JM, Anderson GM, Austin PC, Bronskill SE. Family physicians providing regular care to residents in Ontario long-term care homes: Characteristics and practice patterns. Canadian Family Physician. 2012 Nov 1;58(11):1241-8. 
67. Sloane PD, Zimmerman S, Brown LC, Ives TJ, Walsh JF. Inappropriate medication prescribing in residential care/assisted living facilities. Journal of the American Geriatrics Society. 2002 Jun;50(6):1001-11.

68. Squillace MR, Remsburg RE, Harris-Kojetin LD, Bercovitz A, Rosenoff E, Han B. The national nursing assistant survey: Improving the evidence base for policy initiatives to strengthen the certified nursing assistant workforce. The Gerontologist. 2009 Apr 1;49(2):185-97.

69. Warren JW, Palumbo FB, Fitterman L, Speedie SM. Incidence and characteristics of antibiotic use in aged nursing home patients. Journal of the American Geriatrics Society. 1991 Oct 1;39(10):963-72.

70. Staw BM. The consequences of turnover. Journal of occupational Behaviour. 1980 Oct $1: 253-73$.

71. Robertson JF, Herth KA, Cummings CC. Long-term care: retention of nurses. Journal of Gerontological Nursing. 1994 Nov 1;20(11):4-9.

72. O'Brien-Pallas LG, Tomblin M, Shamian J. Final report: Understanding the costs and outcomes of nurses' turnover in Canadian hospitals. University of Toronto Nursing Health Services Research Unit: Toronto, ON, Canada (2008).

73. Mitchell JB. Physician visits to nursing homes. The Gerontologist. 1982 Feb 1;22(1):45-8.

74. Sloane PD, Huslage K, Kistler CE, Zimmerman S. Optimizing Antibiotic Use in Nursing Homes Through Antibiotic Stewardship. N C Med J. 2016;77(5):324-9.

75. World Health Organization. 2015.

76. Haley RW, Culver DH, White JW, Morgan WM, Emori TG, Munn VP, et al. The efficacy of infection surveillance and control programs in preventing nosocomial infections in US hospitals. Am J Epidemiol. 1985;121(2):182-205.

77. Stuart RL, Marshall C, Orr E, Bennett N, Athan E, Friedman D, et al. Survey of infection control and antimicrobial stewardship practices in Australian residential aged-care facilities. Intern Med J. 2015;45(5):576-80.

78. Thompson BL, Dwyer DM, Ussery XT, Denman S, Vacek P, Schwartz B. Handwashing and glove use in a long-term-care facility. Infect Control Hosp Epidemiol. 1997;18(2):97-103. 
79. Matheï C, Niclaes L, Suetens C, Jans B, Buntinx F. Infections in residents of nursing homes. Infectious disease clinics of North America. 2007 Sep 1;21(3):761-72.

80. Mody L, Saint S, Galecki A, Chen S, Krein SL. Knowledge of evidence-based urinary catheter care practice recommendations among healthcare workers in nursing homes. Journal of the American Geriatrics Society. 2010 Aug;58(8):1532-7.

81. Genao L, Buhr GT. Urinary Tract Infections in Older Adults Residing in Long-Term Care Facilities. Ann Longterm Care. 2012;20(4):33-8.

82. Valderas JM, Starfield B, Sibbald B, Salisbury C, Roland M. Defining comorbidity: implications for understanding health and health services. Ann Fam Med. 2009;7(4):35763.

83. Sakuma M, Morimoto T, Matsui K, Seki S, Kuramoto N, Toshiro J, Murakami J, Fukui T, Saito M, Hiraide A, Bates DW. Epidemiology of potentially inappropriate medication use in elderly patients in Japanese acute care hospitals. Pharmacoepidemiology and drug safety. 2011 Apr 1;20(4):386-92.

84. Nicolle LE, Strausbaugh LJ, Garibaldi RA. Infections and antibiotic resistance in nursing homes. Clin Microbiol Rev. 1996;9(1):1-17.

85. Martin CM. Antibiotic stewardship in long-term care: a call to action. The Consultant Pharmacist@. 2017 May 1;32(5):10-6.

86. M, Mody L. Colonization with multidrug-resistant organisms in nursing homes: scope, importance, and management. Current geriatrics reports. 2015 Mar 1;4(1):87-95.

87. Loeb M, Brazil K, Lohfeld L, McGeer A, Simor A, Stevenson K, et al. Optimizing antibiotics in residents of nursing homes: protocol of a randomized trial. BMC Health Serv Res. 2002;2(1):17.

88. Nicolle LE. Asymptomatic bacteriuria in the elderly. Infectious disease clinics of North America. 1997 Sep 1;11(3):647-62.

89. Ouslander JG, Schapira M, Schnelle JF, Uman G, Fingold S, Tuico E, Nigam JG. Does eradicating bacteriuria affect the severity of chronic urinary incontinence in nursing home residents?. Annals of internal medicine. 1995 May 15;122(10):749-54.

90. Abrutyn E, Mossey J, Berlin JA, Boscia J, Levison M, Pitsakis P, Kaye D. Does asymptomatic bacteriuria predict mortality and does antimicrobial treatment reduce mortality in elderly ambulatory women?. Annals of internal medicine. 1994 May $15 ; 120(10): 827-33$. 
91. Nicolle LE, Bjornson J, Harding GK, MacDonell JA. Bacteriuria in elderly institutionalized men. New England Journal of Medicine. 1983 Dec 8;309(23):1420-5.

92. Nicolle LE, Mayhew WJ, Bryan L. Prospective randomized comparison of therapy and no therapy for asymptomatic bacteriuria in institutionalized elderly women. The American journal of medicine. $1987 \mathrm{Jul}$ 1;83(1):27-33.

93. Pickering TD, Gurwitz JH, Zaleznik D, Noonan JP, Avorn J. The appropriateness of oral fluoroquinolone-prescribing in the long-term care setting. Journal of the American Geriatrics Society. 1994 Jan;42(1):28-32.

94. Nicolle LE. Urinary infections in the elderly: symptomatic or asymptomatic?. International journal of antimicrobial agents. 1999 May 1;11(3-4):265-8.

95. Loeb M, Bentley DW, Bradley S, Crossley K, Garibaldi R, Gantz N, et al. Development of minimum criteria for the initiation of antibiotics in residents of long-term-care facilities: results of a consensus conference. Infect Control Hosp Epidemiol. 2001;22(2):120-4.

96. Jones J, Hunter D. Consensus methods for medical and health services research. BMJ. 1995;311(7001):376-80.

97. Loeb M, Brazil K, Lohfeld L, McGeer A, Simor A, Stevenson K, et al. Effect of a multifaceted intervention on number of antimicrobial prescriptions for suspected urinary tract infections in residents of nursing homes: cluster randomised controlled trial. BMJ. 2005;331(7518):669.

98. European Surveillance of Antimicrobial Consumption (ESAC). Results from the national survey of characteristics of nursing homes. 2009. Available from: http://www.ecdc.europa.eu/en/activities/surveillance/ESAC-Net/publications

99. Najib Ayas MD. A Systematic Review of Research Evidence on:(a) 24-hour Registered Nurse Availability in Long-term Care, and (b) the Relationship between Nurse Staffing and Quality in Long-term Care.

100. Zabarsky TF, Sethi AK, Donskey CJ. Sustained reduction in inappropriate treatment of asymptomatic bacteriuria in a long-term care facility through an educational intervention. Am J Infect Control. 2008;36(7):476-80. 
101. Lim CJ, Kwong M, Stuart RL, Buising KL, Friedman ND, Bennett N, Cheng AC, Peleg AY, Marshall C, Kong DC. Antimicrobial stewardship in residential aged care facilities: need and readiness assessment. BMC infectious diseases. 2014 Dec;14(1):410.

102. Lim CJ, Kwong MW, Stuart RL, Buising KL, Friedman ND, Bennett NJ, Cheng AC, Peleg AY, Marshall C, Kong D. Antibiotic prescribing practice in residential aged care facilitieshealth care providers' perspectives. The Medical Journal of Australia. $2014 \mathrm{Jul}$ $21 ; 201(2): 101-5$.

103. Mylotte JM. Measuring antibiotic use in a long-term care facility. American journal of infection control. 1996 Jun 1;24(3):174-9.

104. Barber ND, Alldred DP, Raynor DK, Dickinson R, Garfield S, Jesson B, Lim R, Savage I, Standage C, Buckle P, Carpenter J. Care homes' use of medicines study: prevalence, causes and potential harm of medication errors in care homes for older people. BMJ Quality \& Safety. 2009 Oct 1;18(5):341-6.

105. Levy SB. Multidrug resistance — a sign of the times. 1998.

106. Campbell RJ, Giljahn L, Machesky K, Cibulskas-White K, Lane LM, Porter K, Paulson JO, Smith FW, McDonald LC. Clostridium difficile infection in Ohio hospitals and nursing homes during 2006. Infection Control \& Hospital Epidemiology. 2009 Jun;30(6):526-33.

107. Rotjanapan P, Dosa D, Thomas KS. Potentially inappropriate treatment of urinary tract infections in two Rhode Island nursing homes. Archives of internal medicine. $2011 \mathrm{Mar}$ $14 ; 171(5): 438-43$.

108. Gruneir A, Bronskill SE, Newman A, Bell CM, Gozdyra P, Anderson GM, et al. Variation in Emergency Department Transfer Rates from Nursing Homes in Ontario, Canada. Healthc Policy. 2016;12(2):76-88.

109. Kahvecioglu D, Ramiah K, McMaughan D, Garfinkel S, McSorley VE, Nguyen QN, Yang M, Pugliese C, Mehr D, Phillips CD. Multidrug-resistant organism infections in US nursing homes: a national study of prevalence, onset, and transmission across care settings, October 1, 2010-December 31, 2011. Infection Control \& Hospital Epidemiology. 2014 Oct;35(S3):S48-55.

110. Van Dijk PT, Mehr DR, Ooms ME, Madsen R, Petroski G, Frijters DH, Margriet Pot A, Ribbe MW. Comorbidity and 1-year mortality risks in nursing home residents. Journal of the American Geriatrics Society. 2005 Apr;53(4):660-5. 
111. Shield RR, Wetle T, Teno J, Miller SC, Welch L. Physicians "Missing in Action": Family Perspectives on Physician and Staffing Problems in End-of-Life Care in the Nursing Home. Journal of the American Geriatrics Society. 2005 Oct;53(10):1651-7.

112. Reimann HA. The misuse of antimicrobics. Med Clin North Am. 1961;45:849-56.

113. So A, Furlong M, Heddini A. Globalisation and antibiotic resistance. 2010.

114. Bartlett JG. A call to arms: the imperative for antimicrobial stewardship. Clinical infectious diseases. 2011 Aug 15;53(suppl_1):S4-7.

115. Dellit TH, Owens RC, McGowan JE, Gerding DN, Weinstein RA, Burke JP, et al. Infectious Diseases Society of America and the Society for Healthcare Epidemiology of America guidelines for developing an institutional program to enhance antimicrobial stewardship. Clin Infect Dis. 2007;44(2):159-77.

116. Slayton RB, Toth D, Lee BY, Tanner W, Bartsch SM, Khader K, Wong K, Brown K, McKinnell JA, Ray W, Miller LG. Vital signs: estimated effects of a coordinated approach for action to reduce antibiotic-resistant infections in health care facilities-United States. MMWR. Morbidity and mortality weekly report. 2015 Aug 7;64(30):826.

117. Kaki R, Elligsen M, Walker S, Simor A, Palmay L, Daneman N. Impact of antimicrobial stewardship in critical care: a systematic review. Journal of antimicrobial chemotherapy. 2011 Apr 2;66(6):1223-30.

118. Nowak MA, Nelson RE, Breidenbach JL, Thompson PA, Carson PJ. Clinical and economic outcomes of a prospective antimicrobial stewardship program. Am J Health Syst Pharm. 2012;69(17):1500-8.

119. Grol R, Grimshaw J. From best evidence to best practice: effective implementation of change in patients' care. Lancet. 2003;362(9391):1225-30.

120. Barlam TF, Cosgrove SE, Abbo LM, MacDougall C, Schuetz AN, Septimus EJ, et al. Implementing an Antibiotic Stewardship Program: Guidelines by the Infectious Diseases Society of America and the Society for Healthcare Epidemiology of America. Clin Infect Dis. 2016;62(10):e51-77.

121. Strausbaugh LJ, Joseph CL. The burden of infection in long-term care. Infection Control \& Hospital Epidemiology. 2000 Oct;21(10):674-9. 
122. Jump RL, Olds DM, Seifi N, Kypriotakis G, Jury LA, Peron EP, et al. Effective antimicrobial stewardship in a long-term care facility through an infectious disease consultation service: keeping a LID on antibiotic use. Infect Control Hosp Epidemiol. 2012;33(12):1185-92.

123. Ibrahim OM, Polk RE. Antimicrobial use metrics and benchmarking to improve stewardship outcomes: methodology, opportunities, and challenges. Infectious Disease Clinics. 2014 Jun 1;28(2):195-214.

124. Thaler R, Sunstein C., Nudge: Improving Decisions About Health, Wealth and Happiness, 2008

125. Yale University Press

126. Michie S, van Stralen MM, West R. The behaviour change wheel: a new method for characterising and designing behaviour change interventions. Implement Sci. 2011;6:42.

127. Schuster MA, McGlynn EA, Brook RH. How good is the quality of health care in the United States?. The Milbank Quarterly. 1998 Dec;76(4):517-63.

128. Malani AN, Brennan BM, Collins CD, Finks J, Pogue JM, Kaye KS. Antimicrobial stewardship practices in Michigan long-term care facilities. infection control \& hospital epidemiology. 2016 Feb;37(2):236-7.

129. Colón-Emeric C, Toles M, Cary MP, Batchelor-Murphy M, Yap T, Song Y, et al. Sustaining complex interventions in long-term care: a qualitative study of direct care staff and managers. Implement Sci. 2016;11:94.

130. Ferlie EB, Shortell SM. Improving the quality of health care in the United Kingdom and the United States: a framework for change. The Milbank Quarterly. 2001 Jun;79(2):281315 .

131. Baur D, Gladstone BP, Burkert F, Carrara E, Foschi F, Döbele S, et al. Effect of antibiotic stewardship on the incidence of infection and colonisation with antibiotic-resistant bacteria and Clostridium difficile infection: a systematic review and meta-analysis. Lancet Infect Dis. 2017;17(9):990-1001.

132. Boyd CM, Darer J, Boult C, Fried LP, Boult L, Wu AW. Clinical practice guidelines and quality of care for older patients with multiple comorbid diseases: implications for pay for performance. JAMA. 2005;294(6):716-24. 
133. Dolan P, Hallsworth M, Halpern D, King D, Vlaev I. MINDSPACE: Influencing behaviour for public policy. 2010.

134. Michie S, Richardson M, Johnston M, Abraham C, Francis J, Hardeman W, et al. The behavior change technique taxonomy (v1) of 93 hierarchically clustered techniques: building an international consensus for the reporting of behavior change interventions. Ann Behav Med. 2013;46(1):81-95.

135. Davey P, Peden C, Charani E, Marwick C, Michie S. Time for action-Improving the design and reporting of behaviour change interventions for antimicrobial stewardship in hospitals: Early findings from a systematic review. Int J Antimicrob Agents. 2015;45(3):203-12.

136. Moullin JC, Sabater-Hernández D, Fernandez-Llimos F, Benrimoj SI. A systematic review of implementation frameworks of innovations in healthcare and resulting generic implementation framework. Health Research Policy and Systems. 2015 Dec;13(1):16.

137. Bond GR, Drake RE, McHugo GJ, Peterson AE, Jones AM, Williams J. Long-term sustainability of evidence-based practices in community mental health agencies. Administration and Policy in Mental Health and Mental Health Services Research. 2014 Mar 1;41(2):228-36.

138. Stirman SW, Kimberly J, Cook N, Calloway A, Castro F, Charns M. The sustainability of new programs and innovations: a review of the empirical literature and recommendations for future research. Implementation Science. 2012 Dec;7(1):17.

139. Septimus EJ, Owens Jr RC. Need and potential of antimicrobial stewardship in community hospitals. Clinical infectious diseases. 2011 Aug 15;53(suppl_1):S8-14.

140. Davey P, Brown E, Charani E, Fenelon L, Gould IM, Holmes A, Ramsay CR, Wiffen PJ, Wilcox M. Interventions to improve antibiotic prescribing practices for hospital inpatients. Cochrane Database Syst Rev. 2013 Jan 1;4(4).

141. Shlaes DM, Gerding DN, John Jr JF, Craig WA, Bornstein DL, Duncan RA, Eckman MR, Farrer WE, Greene WH, Lorian V, Levy S. Society for Healthcare Epidemiology of America and Infectious Diseases Society of America Joint Committee on the Prevention of Antimicrobial Resistance: guidelines for the prevention of antimicrobial resistance in hospitals. Clinical infectious diseases. 1997 Sep 1;25(3):584-99.

142. Atkins D, Eccles M, Flottorp S, Guyatt GH, Henry D, Hill S, Liberati A, O'Connell D, Oxman AD, Phillips B, Schünemann H. Systems for grading the quality of evidence and the strength of recommendations I: critical appraisal of existing approaches The GRADE Working Group. BMC health services research. 2004 Dec;4(1):38. 
143. Network UG. Approach and implications to rating the quality of evidence and strength of recommendations using the GRADE methodology.

144. Alonso-Coello P, Rigau D, Sanabria AJ, Plaza V, Miravitlles M, Martinez L. Quality and strength: the GRADE system for formulating recommendations in clinical practice guidelines. Archivos de Bronconeumología (English Edition). 2013 Jun 1;49(6):261-7.

145. Schünemann HJ, Jaeschke R, Cook DJ, Bria WF, El-Solh AA, Ernst A, Fahy BF, Gould MK, Horan KL, Krishnan JA, Manthous CA. An official ATS statement: grading the quality of evidence and strength of recommendations in ATS guidelines and recommendations. American journal of respiratory and critical care medicine. 2006 Sep $1 ; 174(5): 605-14$.

146. Kavanagh BP. The GRADE system for rating clinical guidelines. PLoS medicine. 2009 Sep 15;6(9):e1000094.

147. Public Health Ontario. Antimicrobial Stewardship Strategies. March 2016. https://www.publichealthontario.ca/en/BrowseByTopic/InfectiousDiseases/AntimicrobialSt ewardshipProgram/Pages/ASP-Strategies.aspx

148. Katz MJ, Gurses AP, Tamma PD, Cosgrove SE, Miller MA, Jump RLP. Implementing Antimicrobial Stewardship in Long-term Care Settings: An Integrative Review Using a Human Factors Approach. Clin Infect Dis. 2017.

149. Marshall LL, Peasah S, Stevens GA. Clostridium difficile Infection in Older Adults: Systematic Review of Efforts to Reduce Occurrence and Improve Outcomes. Consult Pharm. 2017;32(1):24-41.

150. Fleming A, Browne J, Byrne S. The effect of interventions to reduce potentially inappropriate antibiotic prescribing in long-term care facilities: a systematic review of randomised controlled trials. Drugs Aging. 2013;30(6):401-8.

151. Tonkin-Crine SK, Tan PS, van Hecke O, Wang K, Roberts NW, McCullough A, et al. Clinician-targeted interventions to influence antibiotic prescribing behaviour for acute respiratory infections in primary care: an overview of systematic reviews. Cochrane Database Syst Rev. 2017;9:CD012252.

152. Losier M, Ramsey TD, Wilby KJ, Black EK. A Systematic Review of Antimicrobial Stewardship Interventions in the Emergency Department. Ann Pharmacother. 2017;51(9):774-90. 
153. Arnold SR, Straus SE. Interventions to improve antibiotic prescribing practices in ambulatory care. Cochrane Database Syst Rev. 2005(4):CD003539.

154. Kullar R, Yang H, Grein J, Murthy R. A Roadmap to Implementing Antimicrobial Stewardship Principles in Long-Term Care Facilities (LTCFs): Collaboration Between an Acute-Care Hospital and LTCFs. Clin Infect Dis. 2017.

155. Joshi S. Hospital antibiogram: a necessity. Indian journal of medical microbiology. 2010 Oct 1;28(4):277.

156. Furuno JP, Comer AC, Johnson JK, Rosenberg JH, Moore SL, MacKenzie TD, et al. Using antibiograms to improve antibiotic prescribing in skilled nursing facilities. Infect Control Hosp Epidemiol. 2014;35 Suppl 3:S56-61.

157. Chopra TR, Rivard CM. Effective antibiotic stewardship programs at long-term care facilities: A Silver lining in the post-antibiotic era. Ann Long Term Care. 2015;23.

158. Jamtvedt G, Young JM, Kristoffersen DT, O'Brien MA, Oxman AD. Audit and feedback: effects on professional practice and health care outcomes. Cochrane Database Syst Rev. 2006(2):CD000259.

159. Daneman N, Campitelli MA, Giannakeas V, Morris AM, Bell CM, Maxwell CJ, Jeffs L, Austin PC, Bronskill SE. Influences on the start, selection and duration of treatment with antibiotics in long-term care facilities. Canadian Medical Association Journal. 2017 Jun 26;189(25):E851-60.

160. Hux JE, Melady MP, DeBoer D. Confidential prescriber feedback and education to improve antibiotic use in primary care: a controlled trial. CMAJ 1999;161:388-92.

161. Mainous AG III, Hueston WJ, Love MM, Evans ME, Finger R. An evaluation of statewide strategies to reduce antibiotic overuse. Family Medicine 2000;32:22-9. O’Connell 1999

162. Thursky K. The Use of Computerized Decision Support Systems to Support Antimicrobial Stewardship Programs. Antimicrobial Stewardship. 2017 Apr 5;2:99.

163. Schuts EC, Hulscher MEJL, Mouton JW, Verduin CM, Stuart JWTC, Overdiek HWPM, et al. Current evidence on hospital antimicrobial stewardship objectives: a systematic review and meta-analysis. Lancet Infect Dis. 2016;16(7):847-56.

164. Pakyz AL, Moczygemba LR, Wang H, Stevens MP, Edmond MB. An evaluation of the association between an antimicrobial stewardship score and antimicrobial usage. J Antimicrob Chemother. 2015;70(5):1588-91. 
165. Fleet E, Gopal Rao G, Patel B, Cookson B, Charlett A, Bowman C, et al. Impact of implementation of a novel antimicrobial stewardship tool on antibiotic use in nursing homes: a prospective cluster randomized control pilot study. J Antimicrob Chemother. 2014;69(8):2265-73.

166. Koo E, McNamara S, Lansing B, Olmsted RN, Rye RA, Fitzgerald T, et al. Making infection prevention education interactive can enhance knowledge and improve outcomes: Results from the Targeted Infection Prevention (TIP) Study. Am J Infect Control. 2016;44(11):1241-6.

167. Rawson TM, Moore LS, Tivey AM, Tsao A, Gilchrist M, Charani E, et al. Behaviour change interventions to influence antimicrobial prescribing: a cross-sectional analysis of reports from UK state-of-the-art scientific conferences. Antimicrob Resist Infect Control. 2017;6:11.

168. Angunawela II, Diwan VK, Tomson G. Experimental evaluation of the effects of drug information on antibiotic prescribing: a study in outpatient care in an area of Sri Lanka. International Journal of Epidemiology 1991;20:558-64.

169. Avorn J, Soumerai SB. Improving drug-therapy decisions through educational outreach: a randomized controlled trial of academically based "detailing". New England Journal of Medicine 1983;308:1457-63.

170. Schaffner W, Ray WA, Federspiel CF, Miller WO. Improving antibiotic prescribing in office practice: a controlled trial of three educational methods. JAMA 1983;250:1728-32.

171. The Institute for Clinical Evaluative Sciences. Health System Use by Frail Ontario Seniors: An In-Depth Examination of Four Vulnerable Cohorts. 2011. https://www.ices.on.ca/Publications/Atlases-and-Reports/2011/Health-System-Use

172. Haas DW, Bonczar T. Effect of replacing cefotaxime with ceftizoxime in a hospital where penicillin-resistant pneumococcal disease is prevalent. Journal of Antimicrobial Chemotherapy. 1996 Aug 1;38(2):293-9.

173. Bergstrom CT, Lo M, Lipsitch M. Ecological theory suggests that antimicrobial cycling will not reduce antimicrobial resistance in hospitals. Proceedings of the National Academy of Sciences. 2004 Sep 7;101(36):13285-90.

174. Brown EM, Nathwani D. Antibiotic cycling or rotation: a systematic review of the evidence of efficacy. Journal of antimicrobial chemotherapy. 2005 Jan 1;55(1):6-9. 
175. Medina-Walpole AM, McCormick WC. Provider practice patterns in nursing homeacquired pneumonia. J Am Geriatr Soc. 1998;46(2):187-92.

176. Schwartz DN, Abiad H, DeMarais PL, Armeanu E, Trick WE, Wang Y, et al. An educational intervention to improve antimicrobial use in a hospital-based long-term care facility. J Am Geriatr Soc. 2007;55(8):1236-42.

177. Griffin JP, editor. The textbook of pharmaceutical medicine. John Wiley \& Sons; 2009 Oct 15.

178. Goff DA, Bauer KA, Reed EE, Stevenson KB, Taylor JJ, West JE. Is the "low-hanging fruit" worth picking for antimicrobial stewaArdship programs? Clin Infect Dis. 2012;55(4):587-92.

179. Naughton BJ, Mylotte JM, Tayara A. Outcome of nursing home-acquired pneumonia: derivation and application of a practical model to predict 30 day mortality. Journal of the American Geriatrics Society. 2000 Oct 1;48(10):1292-9.

180. Craig P, Dieppe P, Macintyre S, Michie S, Nazareth I, Petticrew M. Developing and evaluating complex interventions: the new Medical Research Council guidance. Bmj. 2008 Sep 29;337:a1655.

181. Monette J, Miller MA, Monette M, Laurier C, Boivin JF, Sourial N, et al. Effect of an educational intervention on optimizing antibiotic prescribing in long-term care facilities. $\mathrm{J}$ Am Geriatr Soc. 2007;55(8):1231-5.

182. Feldstein D, Sloane PD, Feltner C. Antibiotic Stewardship Programs in Nursing Homes: A Systematic Review. J Am Med Dir Assoc. 2017.

183. Naughton BJ, Mylotte JM, Ramadan F, Karuza J, Priore RL. Antibiotic use, hospital admissions, and mortality before and after implementing guidelines for nursing homeacquired pneumonia. J Am Geriatr Soc. 2001;49(8):1020-4.

184. Hecker MT, Aron DC, Patel NP, Lehmann MK, Donskey CJ. Unnecessary use of antimicrobials in hospitalized patients: current patterns of misuse with an emphasis on the antianaerobic spectrum of activity. Arch Intern Med. 2003;163(8):972-8.

185. Lutters M, Vogt-Ferrier NB. Antibiotic duration for treating uncomplicated, symptomatic lower urinary tract infections in elderly women. Cochrane Database Syst Rev. 
186. Gray RP, Malone-Lee J. Review: urinary tract infection in elderly people--time to review management? Age Ageing. 1995;24(4):341-5.

187. Vogel T, Verreault R, Gourdeau M, Morin M, Grenier-Gosselin L, Rochette L. Optimal duration of antibiotic therapy for uncomplicated urinary tract infection in older women: a double-blind randomized controlled trial. CMAJ. 2004;170(4):469-73.

188. Daneman N, Gruneir A, Bronskill SE, Newman A, Fischer HD, Rochon PA, et al. Prolonged antibiotic treatment in long-term care: role of the prescriber. JAMA Intern Med. 2013;173(8):673-82.

189. Patton A, Davey P, Harbarth S, Nathwani D, Sneddon J, Marwick CA. Impact of antimicrobial stewardship interventions on Clostridium difficile infection and clinical outcomes: segmented regression analyses. J Antimicrob Chemother. 2017.

190. Jury LA, Tomas M, Kundrapu S, Sitzlar B, Donskey CJ. A Clostridium difficile infection (CDI) stewardship initiative improves adherence to practice guidelines for management of CDI. Infect Control Hosp Epidemiol. 2013;34(11):1222-4.

191. Welch HK, Nagel JL, Patel TS, Gandhi TN, Chen B, De Leon J, et al. Effect of an antimicrobial stewardship intervention on outcomes for patients with Clostridium difficile infection. Am J Infect Control. 2016;44(12):1539-43.

192. Cohen SH, Gerding DN, Johnson S, Kelly CP, Loo VG, McDonald LC, et al. Clinical practice guidelines for Clostridium difficile infection in adults: 2010 update by the society for healthcare epidemiology of America (SHEA) and the infectious diseases society of America (IDSA). Infect Control Hosp Epidemiol. 2010;31(5):431-55.

193. Wagner B, Filice GA, Drekonja D, Greer N, MacDonald R, Rutks I, Butler M, Wilt TJ. Antimicrobial stewardship programs in inpatient hospital settings: a systematic review. Infection Control \& Hospital Epidemiology. 2014 Oct;35(10):1209-28.

194. Fitch K, Bernstein SJ, Aguilar MD, Burnand B, LaCalle JR. The RAND/UCLA appropriateness method user's manual. RAND CORP SANTA MONICA CA; 2001 Jan.

195. Dalkey N, Helmer O. An experimental application of the Delphi method to the use of experts. Management science. 1963 Apr;9(3):458-67.

196. Moehring RW, Anderson DJ, Cochran RL, Hicks LA, Srinivasan A, Dodds Ashley ES, et al. Expert Consensus on Metrics to Assess the Impact of Patient-Level Antimicrobial Stewardship Interventions in Acute-Care Settings. Clin Infect Dis. 2017;64(3):377-83. 
197. Kahan JP, Morton SC, Farris HH, Kominski GF, Donovan AJ. Panel processes for revising relative values of physician work. A pilot study. Med Care. 1994;32(11):1069-85.

198. Habibi A, Sarafrazi A, Izadyar S. Delphi technique theoretical framework in qualitative research. The International Journal of Engineering and Science. 2014;3(4):8-13.

199. Morris AM, Brener S, Dresser L, Daneman N, Dellit TH, Avdic E, Bell CM. Use of a structured panel process to define quality metrics for antimicrobial stewardship programs. Infection Control \& Hospital Epidemiology. 2012 May;33(5):500-6.

200. Dresser LD, Bell CM, Steinberg M, Ferguson ND, Lapinsky S, Lazar N, Murphy P, Singh JM, Morris AM. Use of a structured panel process to define antimicrobial prescribing appropriateness in critical care. Journal of Antimicrobial Chemotherapy. 2017 Sep 26;73(1):246-9.

201. Boyd H, McKernon S, Mullin B, Old A. Improving healthcare through the use of co-design. N Z Med J. 2012;125(1357):76-87.

202. Kennedy CC, Thabane L, Ioannidis G, Adachi JD, Papaioannou A, Investigators V. Implementing a knowledge translation intervention in long-term care: feasibility results from the Vitamin D and Osteoporosis Study (ViDOS). J Am Med Dir Assoc. 2014;15(12):943-5.

203. Freemantle F, Harvey EL, Wolf, F et al.Printed educational materials to improve the behaviour of health care professionals and patient outcomes (Cochrane Review)

204. The Cochrane Library, Update Software, Oxford (1998) issue 3

205. Thomas DR, Bennett RG, Laughon BE, Greenough WB, Bartlett JG. Postantibiotic colonization with Clostridium difficile in nursing home patients. Journal of the American Geriatrics Society. 1990 Apr;38(4):415-20.

206. McElligott M, Welham G, Pop-Vicas A, Taylor L, Crnich CJ. Antibiotic Stewardship in Nursing Facilities. Infect Dis Clin North Am. 2017;31(4):619-38.

207. Katz PR, Karuza J. Physician practice in the nursing home: missing in action or misunderstood. J Am Geriatr Soc. 2005;53(10):1826-8.

208. Zimmerman S, Sloane PD, Bertrand R, Olsho LE, Beeber A, Kistler C, et al. Successfully reducing antibiotic prescribing in nursing homes. J Am Geriatr Soc. 2014;62(5):907-12.

209. Renz SM, Boltz MP, Wagner LM, Capezuti EA, Lawrence TE. Examining the feasibility and 
utility of an SBAR protocol in long-term care. Geriatr Nurs. 2013;34(4):295-301.

210. Brink AJ, Messina AP, Feldman C, Richards GA, Becker PJ, Goff DA, Bauer KA, Nathwani D, Van den Bergh D. Antimicrobial stewardship across 47 South African hospitals: an implementation study. The Lancet Infectious Diseases. 2016 Sep $1 ; 16(9): 1017-25$.

211. Gunter TD, Terry NP. The emergence of national electronic health record architectures in the United States and Australia: models, costs, and questions. J Med Internet Res. 2005;7(1):e3.

212. Kruse CS, Mileski M, Vijaykumar AG, Viswanathan SV, Suskandla U, Chidambaram Y. Impact of Electronic Health Records on Long-Term Care Facilities: Systematic Review. JMIR Med Inform. 2017;5(3):e35.

213. Broughton W, Lashlee H, Marcum C, Wilson GM. Health information technology: a new world of nursing homes. J Gerontol Geriatric Res. 2013;2:122.

214. McMaughan DK, Nwaiwu O, Zhao H, Frentzel E, Mehr D, Imanpour S, Garfinkel S, Phillips CD. Impact of a decision-making aid for suspected urinary tract infections on antibiotic overuse in nursing homes. BMC geriatrics. 2016 Dec;16(1):81.

215. Ajami S, Bagheri-Tadi T. Barriers for Adopting Electronic Health Records (EHRs) by Physicians. Acta Inform Med. 2013;21(2):129-34.

216. Kruse CS, Mileski M, Alaytsev V, Carol E, Williams A. Adoption factors associated with electronic health record among long-term care facilities: a systematic review. BMJ Open. 2015;5(1):e006615.

217. Davis DA, Taylor-Vaisey A. Translating guidelines into practice: a systematic review of theoretic concepts, practical experience and research evidence in the adoption of clinical practice guidelines. Canadian Medical Association Journal. 1997 Aug 15;157(4):408-16.

218. File TM, Garau J, Blasi F, Chidiac C, Klugman K, Lode H, et al. Guidelines for empiric antimicrobial prescribing in community-acquired pneumonia. Chest. 2004;125(5):1888-901.

219. Lyons H. Solutions by consensus. 1981. Health and Social Services Journal 2, 1515-1516.

220. Leape LL, Park RE, Kahan JP, Brook RH. Group judgments of appropriateness: the effect of panel composition. Qual Assur Health Care. 1992;4(2):151-9. 
221. Coulter I, Adams A, Shekelle P. Impact of varying panel membership on ratings of appropriateness in consensus panels: a comparison of a multi- and single disciplinary panel. Health Serv Res. 1995;30(4):577-91.

222. Bowers JM, Benford S, editors. New York: Eslevier Science Pub.Co; 1991. Studies in computer supported cooperative work: theory, practice, and design.

223. Munyisia E, Yu P, Hailey D. The effect of an electronic health record system on nursing staff time in a nursing home: a longitudinal cohort study. Australas Med J. 2014;7(7):285-93. 


\section{Appendix A. Background Package \\ Brief Literature Summary}

\section{Background:}

Nursing homes (typically known as long-term care facilities in Canada) are an important health care setting as they typically care for some of the most vulnerable and complex older adults in society. Older adult populations are increasing rapidly and are expected to continue growing over the next few decades. In Canada, older adults (aged 65 and over) are anticipated to account for $20.1 \%$ of the population by 2024 and $25 \%$ of the population by $2036 .{ }^{1-3}$ While not all older adults require nursing home care, they are provincially provided to all individuals who need rehabilitation from surgery, illness and injury. There are over 600 nursing homes located in Ontario, with over 77,000 beds. There are approximately 255,000 nursing home beds in Canada, a number that is expected to double by $2035 .^{4-6}$

Nursing home residents are highly susceptible to infection. This is because residents represent the oldest and most frail cohort within the overall population and reside in an environment highly prevalent with infectious agents. ${ }^{7}$ Previous research has demonstrated that approximately 3-15\% of nursing home residents will acquire an infection during their stay in a facility. ${ }^{6}$ Antibiotics are the most commonly prescribed medication in nursing homes, accounting for $40 \%$ of all prescribed medications; however, most (50-75\%) antibiotic prescriptions in nursing homes may be considered inappropriate. ${ }^{8}$ Inappropriate antibiotic use can take various forms including (but not limited to) the prescribing process, such as the choice of antibiotic (broad-spectrum vs. narrow-spectrum); common prescribing practices, such as the use of prophylaxis; the availability of diagnostic tools and the need for empiric antibiotic prescriptions (prescribing antibiotics without identifying the infecting organism). ${ }^{9-12}$ Inappropriate antibiotic use endangers the individual, the overall nursing home, and the community. ${ }^{9}$ Of particular worry is that inappropriate antibiotics increase a resident's likelihood of experiencing adverse drug events.

An even greater concern is the relationship between inappropriate antibiotic use and the spread of antibiotic resistance. Antibiotic use is the driving force for the promotion of antibiotic resistant bacteria. It is estimated that 1 in 3 nursing home residents will acquire a multidrug-resistant organism during their stay in a facility. ${ }^{8,10,11}$ Antibiotic resistance is directly linked to increased fatality, length of hospital stay, and cost of treatment. ${ }^{12}, 13$ Thus, reducing inappropriate antibiotic use is critical for improving patient outcomes and preventing the spread of antibiotic resistance. $^{14,15}$

Diagnosing infections in nursing homes, and treating them appropriately, is a challenging task even for the most experienced health care providers. Resident conditions, such as comorbidity, polypharmacy, and frailty, can potentially disrupt the process of identifying an infecting organism and discerning the cause of symptoms, such as those as a result of infection or medication side effects. ${ }^{16-18}$

Antimicrobial Stewardship Programs (ASPs) are ongoing institutional initiatives and interventions to optimize the use of antibiotics and improve patient outcomes, whilst reducing inappropriate antibiotics, adverse events, and overall cost of therapy. ${ }^{9}$ ASPs have been 
effectively implemented and reviewed in acute care hospital and ambulatory settings, but efforts for implementing ASPs in nursing homes are mostly unknown. ${ }^{19,20}$ Infectious Diseases Society of America and the Centers for Disease Control and Prevention recommend implementation of ASPs in nursing homes; however, little guidance is provided for selecting and initiating such programs. Selecting an ASP is a challenging task, as many strategies exist (Table 1) and the evidence based from the research literature on each is mixed. ${ }^{10,11,18,21-26}$ Therefore, clarification is needed to support nursing homes in optimizing their selection of ASPs and to describe the resource requirements for implementing different ASPs.

Our goal for this modified Delphi panel (supported by a larger grant from the Canadian Frailty Network) is to utilize a two-part survey to evaluate ASPs according to five (5) criteria: scientific merit (quality of the science), impact (capacity for change), feasibility (resource demand), accountability (public reporting), and importance for nursing homes. These criteria were chosen because, in combination, they provide a rounded evaluation of the interventions and have been successfully used in previous modified Delphi panels. ${ }^{27}$

The first round of the survey is provided concurrently with this background package. Participants will evaluate the use of the selected criteria (scientific merit, impact, etc.), followed by evaluation of the ASPs. Discussion of the results from the first round of the survey and completion of the second round of the survey will be take place on the day of the panel meeting (March 20, 2018). Following a discussion of the ASPs, resourcing implications for each ASP will be discussed and strategies will be prioritized.

The goal of this structured process is to be able to provide interested nursing homes with 2 tools for implementing effective ASPs:

1. A list of ASPs deemed applicable and necessary for nursing homes;

2. A list of associated resources required for implementation of interventions; and

3. A list of ASPs prioritized by their evaluation and resource-demand. 


\section{Systematic Reviews:}

The following is a brief summary of some systematic reviews describing ASP implementation in acute care hospital, ambulatory, emergency department, and nursing homes settings. The reviews were identified through PubMed, Medline, and librarian assistance from the Sidney Liswood Health Sciences Library at Mount Sinai Hospital, Toronto (see Appendix for search strategy).

This is not an exhaustive review, and should only be used as a supplement to your own experience and expertise.

Reference: Baur D, Gladstone BP, Burkert F, Carrara E, Foschi F, Döbele S, Tacconelli E. Effect of antibiotic stewardship on the incidence of infection and colonisation with antibioticresistant bacteria and Clostridium difficile infection: a systematic review and meta-analysis. The Lancet Infectious Diseases 17.9 (2017): 990-1001.

Summary: Systematic review and meta-analysis of ASPs in hospital (inpatient) settings for reducing incidence of antibiotic-resistant infections. A total of 32 studies were included in the meta-analysis. This review found three ASP strategies significantly effective for reducing antibiotic-resistant infections: antibiotic cycling, audit and feedback, and formulary restriction. Two ASP strategies were not significantly effective: guideline implementation, and focus on single antibiotic classes.

Reference: Katz, MJ, Gurses AP, Tamma PD, Cosgrove SE, Miller MA, \& Jump RL. Implementing Antimicrobial Stewardship in Long-term Care Settings: An Integrative Review Using a Human Factors Approach. Clinical Infectious Diseases 65.11 (2017): 1943-1951.

Summary: Integrative review of three ASP intervention strategies in long-term care facilities (nursing homes): human factors engineering approach, education, and multifaceted interventions. This review identified 20 studies. Interdisciplinary educational interventions, incorporating prescriber feedback was associated with reduced overall consumption of antibiotics without increased rates of adverse events. Thus, highlighting the importance of improved interdisciplinary communication.

Reference: Marshall LL, Samuel P, Gregg AS. Clostridium difficile infection in older adults: systematic review of efforts to reduce occurrence and improve outcomes. The Consultant Pharmacist@ 32.1 (2017): 24-41.

Summary: Systematic review of ASP interventions in community, assisted living, nursing home, and hospital settings. 105 articles were included for full review. Primary outcomes: influence of intervention for preventing, reducing, managing, and improving outcomes. Particular interest: intervention effectiveness for reduced rates of Clostridium difficile infection (CDI). Effective interventions: formulary restriction for broad-spectrum antibiotics for all infections except CDI; improved infection, prevention, and control programs (IPCs); audit and feedback; prescriber education.

Reference: Nicolle LE. Antimicrobial stewardship in long term care facilities: what is effective? Antimicrobial resistance and infection control 3.1 (2014): 6.

Summary: Systematic review of ASP interventions in nursing homes. Identified four studies: two were randomized control trials (RCTs) and two were multifaceted interventions. ASPs should have a set of core elements that are customizable for specific interventions. Engaging specialized physicians, such as infectious disease (ID) consultants and internists, was associated with larger and more consistent improvements. Effective interventions: targeting antibiotic 
treatment of asymptomatic urinary tract infections (UTIs). Less effective interventions: education, local guidelines, and prescriber feedback.

Reference: Fleming A, John B, Stephen B. The effect of interventions to reduce potentially inappropriate antibiotic prescribing in long-term care facilities: a systematic review of randomised controlled trials. Drugs \& aging 30.6 (2013): 401-408.

Summary: Systematic review of ASP interventions in nursing homes. Identified four studies: three were cluster-RCTs (cRCTS) and one was an RCT. Multifaceted interventions incorporating small-group educational sessions are acceptable to nurses and physicians. Development of guidelines utilizing local consensus procedures may provide for a more effective ASP intervention.

Reference: Tonkin-Crine S, et al. Clinician-targeted interventions to reduce antibiotic prescribing for acute respiratory infections in primary care: an overview of systematic reviews. (2016).

Summary: Overview synthesis of systematic reviews of clinician-targeted ASPs in primary care. Assessed interventions for improving antibiotics for treatment of acute respiratory infections (ARIs). Eight reviews were included in the qualitative analysis. Moderate evidence for reducing antibiotic use was established for three interventions: point-of-care tests (tool for immediate diagnostic information), guided management of procalcitonin, and shared decision-making. Conclusions could not be drawn from three interventions: multifaceted, clinical education, and patient education.

Reference: Losier, M, et al. A Systematic Review of Antimicrobial Stewardship Interventions in the Emergency Department. Annals of Pharmacotherapy 51.9 (2017): 774-790.

Summary: Systematic review of ASPs in emergency department settings. Identified 43 studies; most interventions targeted patient and provider education, and guideline development. Several significant improvements were found: adherence to guidelines, antibiotic appropriateness, and overall decreased antibiotic use. Organizing a multifaceted intervention is challenging; difficult to determine which intervention combinations are optimal.

Reference: Arnold SR, Sharon ES. Interventions to improve antibiotic prescribing practices in ambulatory care. The Cochrane Library (2005).

Summary: Systematic review of ASPs in ambulatory care. Outcomes of interest: improved selection, dose, and duration of antibiotics, and reduced incidence of resistant pathogens. 39 studies were identified and evaluated several intervention strategies: education materials for prescribers, audit and feedback, educational meetings and outreach visits, healthcare system changes, reminders, patient-directed, and multifaceted interventions. Printed educational material and audit and feedback were not very effective. Education was more effective when interactive. Mixed results for outreach visits and reminders. Multifaceted interventions that included physician, patient, and public education were the most effective for reducing inappropriate antibiotics. 


\section{Appendix B. Survey 1 (paper version)}

\section{ASP Evaluative Criteria}

In the following pages, you will be required to evaluate candidate ASPs in nursing homes across several criteria. In the background document, we explained a brief rationale for why each of these criteria were chosen.

Please review the following criteria and, if necessary, provide comments where indicated:

\section{Criteria}

Instructions: Please rate your agreement in applying the criteria below in the evaluation of ASPs. Please evaluate each of the following criterion on a 9-point scale (1 - Strongly Disagree, 9 - Strongly Agree)

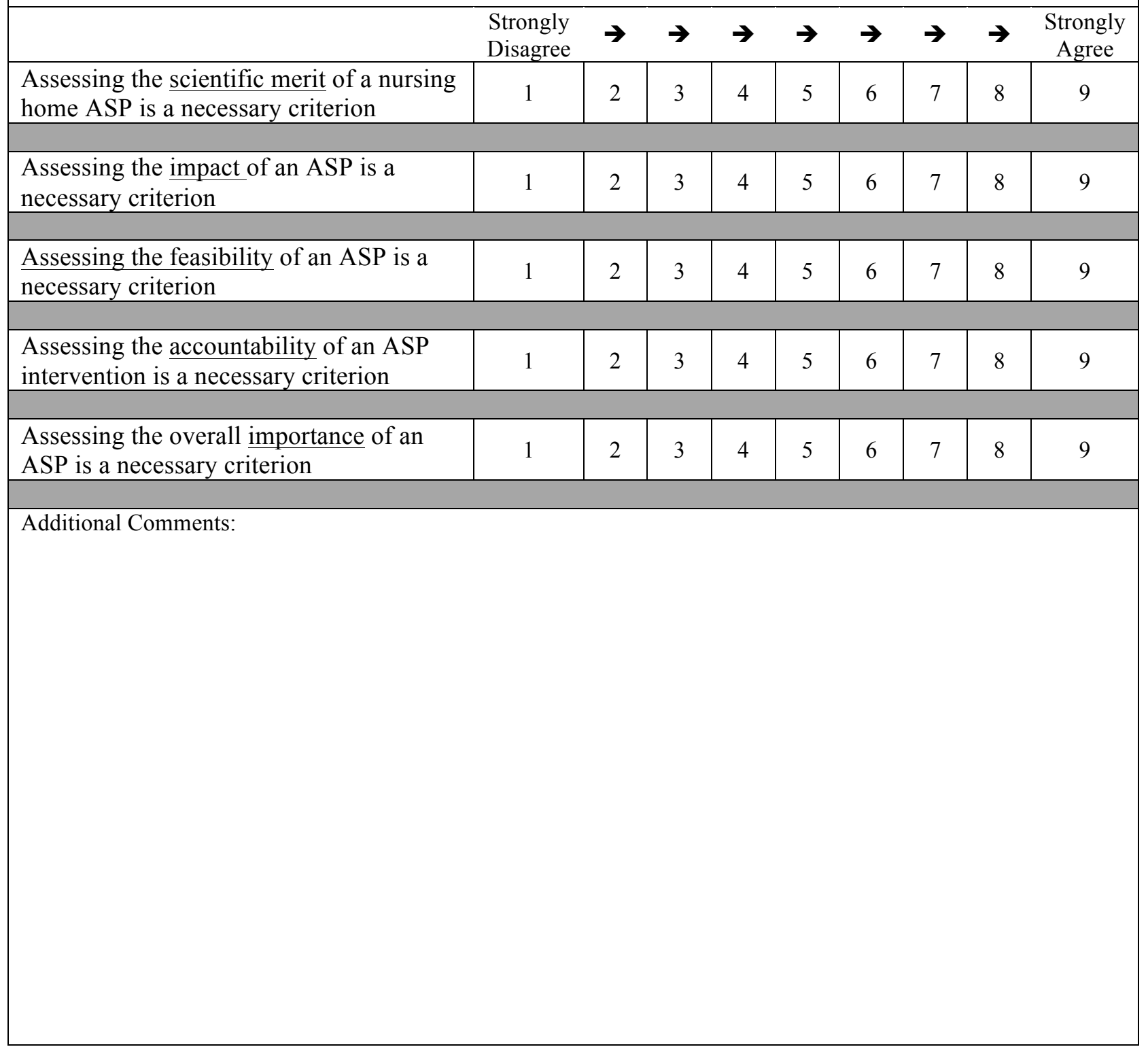




\section{Antibiograms}

Description: A tool for summarizing susceptibility of bacteria to antibiotics at a given point in time.

\section{Antibiograms}

Instructions: Please rate your agreement in applying each of the following criteria on a 9-point scale (1 - Strongly Disagree, 9 - Strongly Agree)

\begin{tabular}{|c|c|c|c|c|c|c|c|c|c|}
\hline & $\begin{array}{l}\text { Strongly } \\
\text { Disagree }\end{array}$ & $\rightarrow$ & $\rightarrow$ & $\rightarrow$ & & $\rightarrow$ & $\rightarrow$ & $\rightarrow$ & $\begin{array}{l}\text { Strongly } \\
\text { Agree }\end{array}$ \\
\hline $\begin{array}{l}\text { This intervention has a high degree of } \\
\text { scientific merit }\end{array}$ & 1 & 2 & 3 & 4 & 5 & 6 & 7 & 8 & 9 \\
\hline $\begin{array}{l}\text { Such an intervention has the potential for } \\
\text { significant impact in nursing homes }\end{array}$ & 1 & 2 & 3 & 4 & 5 & 6 & 7 & 8 & 9 \\
\hline $\begin{array}{l}\text { This intervention is feasible for nursing } \\
\text { homes }\end{array}$ & 1 & 2 & 3 & 4 & 5 & 6 & 7 & 8 & 9 \\
\hline $\begin{array}{l}\text { Such an intervention is useful } \\
\text { for accountability purposes }\end{array}$ & 1 & 2 & 3 & 4 & 5 & 6 & 7 & 8 & 9 \\
\hline $\begin{array}{l}\text { Implementation of this intervention in } \\
\text { nursing homes is important }\end{array}$ & 1 & 2 & 3 & 4 & 5 & 6 & 7 & 8 & 9 \\
\hline
\end{tabular}

Additional Comments: 


\section{Antibiotic Cycling}

Description: Repeated exchange, or cycling, of antibiotics of a certain class with those of another class. Eventual return to the original antibiotic class and continued cycling.

\section{Antibiotic Cycling}

Instructions: Please rate your agreement in applying each of the following criteria on a 9-point scale (1 - Strongly Disagree, 9 - Strongly Agree)

\begin{tabular}{|l|c|c|c|c|c|c|c|c|c|c|}
\hline & $\begin{array}{l}\text { Strongly } \\
\text { Disagree }\end{array}$ & $\rightarrow$ & $\rightarrow$ & $\rightarrow$ & $\rightarrow$ & $\rightarrow$ & $\rightarrow$ & \multicolumn{1}{c}{$\begin{array}{c}\text { Strongly } \\
\text { Agree }\end{array}$} \\
\hline $\begin{array}{l}\text { This intervention has a high degree of } \\
\text { scientific merit }\end{array}$ & 1 & 2 & 3 & 4 & 5 & 6 & 7 & 8 & 9 \\
\hline
\end{tabular}

\begin{tabular}{|c|c|c|c|c|c|c|c|c|c|}
\hline $\begin{array}{l}\text { Such an intervention has the potential for } \\
\text { significant impact in nursing homes }\end{array}$ & 1 & 2 & 3 & 4 & 5 & 6 & 7 & 8 & 9 \\
\hline $\begin{array}{l}\text { This intervention is feasible for nursing } \\
\text { homes }\end{array}$ & 1 & 2 & 3 & 4 & 5 & 6 & 7 & 8 & 9 \\
\hline $\begin{array}{l}\text { Such an intervention is useful } \\
\text { for accountability purposes }\end{array}$ & 1 & 2 & 3 & 4 & 5 & 6 & 7 & 8 & 9 \\
\hline $\begin{array}{l}\text { Implementation of this intervention in } \\
\text { nursing homes is important }\end{array}$ & 1 & 2 & 3 & 4 & 5 & 6 & 7 & 8 & 9 \\
\hline
\end{tabular}

Additional Comments: 


\section{Audit and Feedback}

Description: Summarization of a clinician's prescribing performance and recommendations for adjustment.

\section{Audit and Feedback}

Instructions: Please rate your agreement in applying each of the following criteria on a 9-point scale (1 - Strongly Disagree, 9 - Strongly Agree)

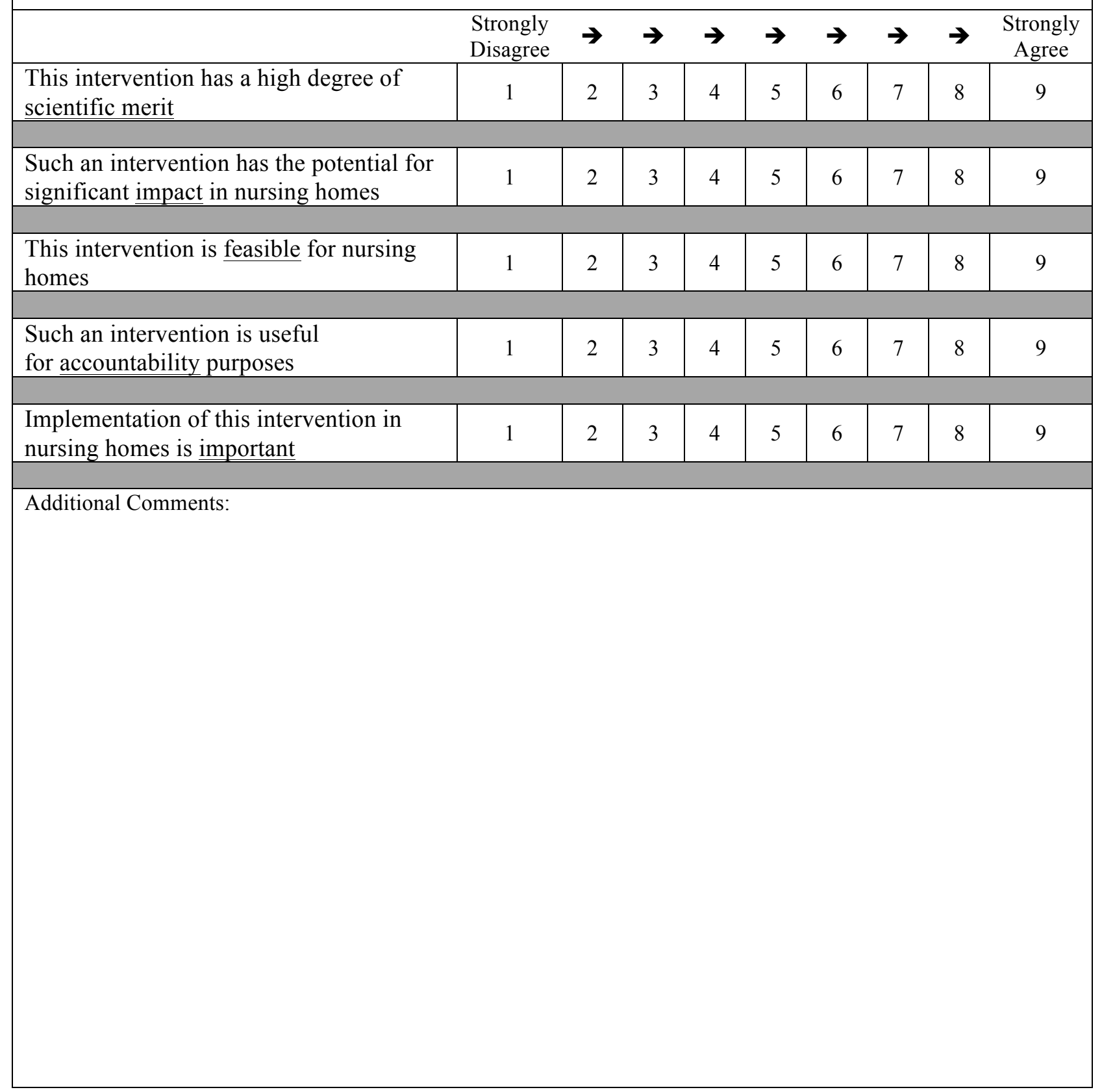




\section{Clinical Decision Support Systems}

Description: Tool (computerized) for providing clinicians with relevant information for arriving at correct diagnosis and selection of antibiotics.

\section{Clinical Decision Support Systems}

Instructions: Please rate your agreement in applying each of the following criteria on a 9-point scale (1 - Strongly Disagree, 9 - Strongly Agree)

\begin{tabular}{|c|c|c|c|c|c|c|c|c|c|}
\hline & $\begin{array}{l}\text { Strongly } \\
\text { Disagree }\end{array}$ & $\rightarrow$ & $\rightarrow$ & $\rightarrow$ & $\rightarrow$ & $\rightarrow$ & $\rightarrow$ & $\rightarrow$ & $\begin{array}{l}\text { Strongly } \\
\text { Agree }\end{array}$ \\
\hline $\begin{array}{l}\text { This intervention has a high degree of } \\
\text { scientific merit }\end{array}$ & 1 & 2 & 3 & 4 & 5 & 6 & 7 & 8 & 9 \\
\hline $\begin{array}{l}\text { Such an intervention has the potential for } \\
\text { significant impact in nursing homes }\end{array}$ & 1 & 2 & 3 & 4 & 5 & 6 & 7 & 8 & 9 \\
\hline $\begin{array}{l}\text { This intervention is feasible for nursing } \\
\text { homes }\end{array}$ & 1 & 2 & 3 & 4 & 5 & 6 & 7 & 8 & 9 \\
\hline $\begin{array}{l}\text { Such an intervention is useful } \\
\text { for accountability purposes }\end{array}$ & 1 & 2 & 3 & 4 & 5 & 6 & 7 & 8 & 9 \\
\hline $\begin{array}{l}\text { Implementation of this intervention in } \\
\text { nursing homes is important }\end{array}$ & 1 & 2 & 3 & 4 & 5 & 6 & 7 & 8 & 9 \\
\hline
\end{tabular}

Additional Comments: 


\section{De-escalation}

Description: Readjustment of antibiotics following culture results: transitioning from broadspectrum to narrow-spectrum antibiotics, conversion of empiric to pathogen-directed therapy, discontinuation of unnecessary antibiotics.

\section{De-escalation}

Instructions: Please rate your agreement in applying each of the following criteria on a 9-point scale (1 - Strongly Disagree, 9 - Strongly Agree)

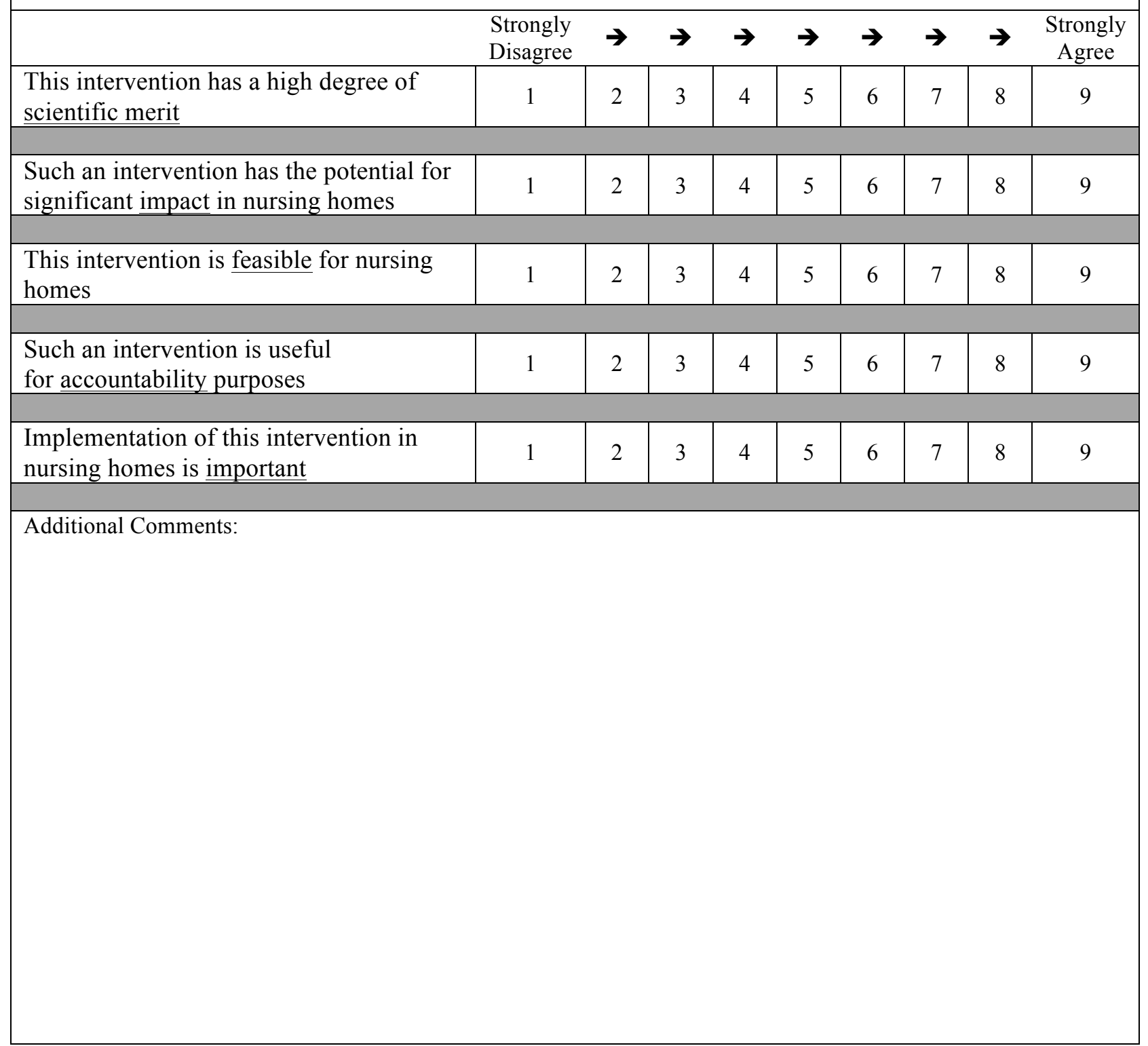




\section{Education}

Description: Education of NH staff (nurses, physicians, HCPs, etc.) and patients/families to better their understanding of bacterial resistance, share knowledge of ASP principles, and improve antibiotic prescribing.

\section{Education}

Instructions: Please rate your agreement in applying each of the following criteria on a 9-point scale (1 - Strongly Disagree, 9 - Strongly Agree)

\begin{tabular}{|c|c|c|c|c|c|c|c|c|c|}
\hline & $\begin{array}{l}\text { Strongly } \\
\text { Disagree }\end{array}$ & $\rightarrow$ & $\rightarrow$ & $\rightarrow$ & $\rightarrow$ & $\rightarrow$ & $\rightarrow$ & $\rightarrow$ & $\begin{array}{c}\text { Strongly } \\
\text { Agree }\end{array}$ \\
\hline $\begin{array}{l}\text { This intervention has a high degree of } \\
\text { scientific merit }\end{array}$ & 1 & 2 & 3 & 4 & 5 & 6 & 7 & 8 & 9 \\
\hline $\begin{array}{l}\text { Such an intervention has the potential for } \\
\text { significant impact in nursing homes }\end{array}$ & 1 & 2 & 3 & 4 & 5 & 6 & 7 & 8 & 9 \\
\hline $\begin{array}{l}\text { This intervention is feasible for nursing } \\
\text { homes }\end{array}$ & 1 & 2 & 3 & 4 & 5 & 6 & 7 & 8 & 9 \\
\hline $\begin{array}{l}\text { Such an intervention is useful } \\
\text { for accountability purposes }\end{array}$ & 1 & 2 & 3 & 4 & 5 & 6 & 7 & 8 & 9 \\
\hline $\begin{array}{l}\text { Implementation of this intervention in } \\
\text { nursing homes is important }\end{array}$ & 1 & 2 & 3 & 4 & 5 & 6 & 7 & 8 & 9 \\
\hline
\end{tabular}

Additional Comments: 


\section{Formulary Automatic Substitution}

Description: Automatic substitution of certain drugs by pharmacists without the need for prescriber consultation.

\section{Formulary Automatic Substitution}

Instructions: Please rate your agreement in applying each of the following criteria on a 9-point scale (1 - Strongly Disagree, 9 - Strongly Agree)

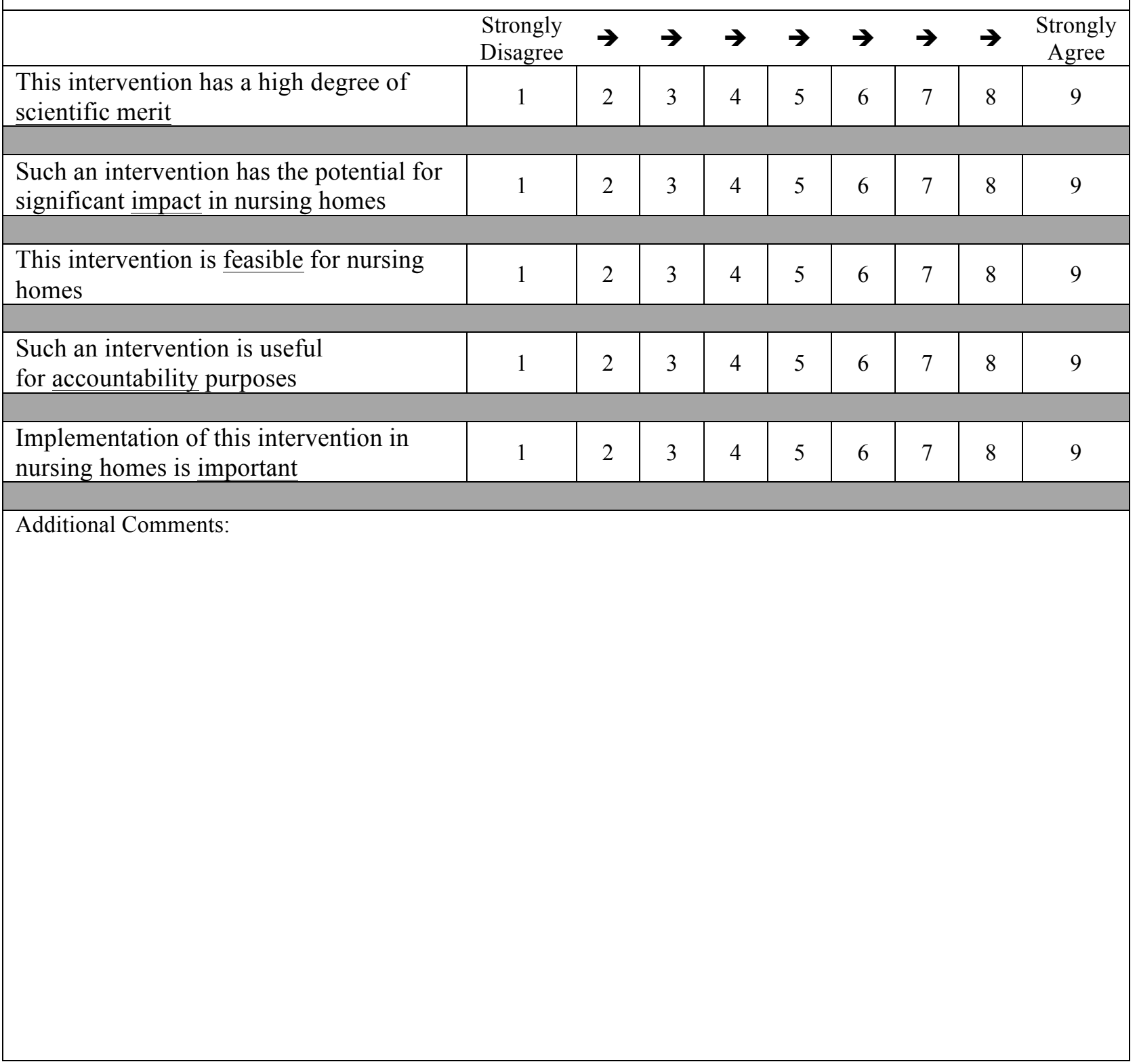




\section{Formulary Restriction}

Description: Restriction of certain antibiotics and guidance for their use due to risk of resistance/ADEs, over/misuse, broad spectrum, expense.

\section{Formulary Restriction}

Instructions: Please rate your agreement in applying each of the following criteria on a 9-point scale (1 - Strongly Disagree, 9 - Strongly Agree)

\begin{tabular}{|c|c|c|c|c|c|c|c|c|c|}
\hline & $\begin{array}{l}\text { Strongly } \\
\text { Disagree }\end{array}$ & $\rightarrow$ & $\rightarrow$ & $\Rightarrow$ & $\rightarrow$ & $\rightarrow$ & $\rightarrow$ & $\rightarrow$ & $\begin{array}{c}\text { Strongly } \\
\text { Agree }\end{array}$ \\
\hline $\begin{array}{l}\text { This intervention has a high degree of } \\
\text { scientific merit }\end{array}$ & 1 & 2 & 3 & 4 & 5 & 6 & 7 & 8 & 9 \\
\hline & & & & & & & & & \\
\hline $\begin{array}{l}\text { Such an intervention has the potential for } \\
\text { significant impact in nursing homes }\end{array}$ & 1 & 2 & 3 & 4 & 5 & 6 & 7 & 8 & 9 \\
\hline $\begin{array}{l}\text { This intervention is feasible for nursing } \\
\text { homes }\end{array}$ & 1 & 2 & 3 & 4 & 5 & 6 & 7 & 8 & 9 \\
\hline $\begin{array}{l}\text { Such an intervention is useful } \\
\text { for accountability purposes }\end{array}$ & 1 & 2 & 3 & 4 & 5 & 6 & 7 & 8 & 9 \\
\hline $\begin{array}{l}\text { Implementation of this intervention in } \\
\text { nursing homes is important }\end{array}$ & 1 & 2 & 3 & 4 & 5 & 6 & 7 & 8 & 9 \\
\hline
\end{tabular}

Additional Comments: 


\section{Formulary Review}

Description: Institutional review of available antibiotics.

\section{Formulary Review}

Instructions: Please rate your agreement in applying each of the following criteria on a 9-point scale (1 - Strongly Disagree, 9 - Strongly Agree)

\begin{tabular}{|c|c|c|c|c|c|c|c|c|c|}
\hline & $\begin{array}{l}\text { Strongly } \\
\text { Disagree }\end{array}$ & $\rightarrow$ & & $\rightarrow$ & $\rightarrow$ & $\rightarrow$ & $\rightarrow$ & & $\begin{array}{l}\text { Strongly } \\
\text { Agree }\end{array}$ \\
\hline $\begin{array}{l}\text { This intervention has a high degree of } \\
\text { scientific merit }\end{array}$ & 1 & 2 & 3 & 4 & 5 & 6 & 7 & 8 & 9 \\
\hline $\begin{array}{l}\text { Such an intervention has the potential for } \\
\text { significant impact in nursing homes }\end{array}$ & 1 & 2 & 3 & 4 & 5 & 6 & 7 & 8 & 9 \\
\hline $\begin{array}{l}\text { This intervention is feasible for nursing } \\
\text { homes }\end{array}$ & 1 & 2 & 3 & 4 & 5 & 6 & 7 & 8 & 9 \\
\hline $\begin{array}{l}\text { Such an intervention is useful } \\
\text { for accountability purposes }\end{array}$ & 1 & 2 & 3 & 4 & 5 & 6 & 7 & 8 & 9 \\
\hline $\begin{array}{l}\text { Implementation of this intervention in } \\
\text { nursing homes is important }\end{array}$ & 1 & 2 & 3 & 4 & 5 & 6 & 7 & 8 & 9 \\
\hline
\end{tabular}

Additional Comments: 


\section{Guidelines for Empiric Prescribing}

Description: Development of standardized, multidisciplinary, evidence-based guidelines to aid prescribers in their initiation of antibiotic therapy.

\section{Guidelines for Empiric Prescribing}

Instructions: Please rate your agreement in applying each of the following criteria on a 9-point scale ( 1 - Strongly Disagree, 9 - Strongly Agree)

\begin{tabular}{|l|c|c|c|c|c|c|c|c|c|c|c|}
\hline & $\begin{array}{c}\text { Strongly } \\
\text { Disagree }\end{array}$ & $\rightarrow$ & $\rightarrow$ & $\rightarrow$ & $\rightarrow$ & $\rightarrow$ & $\rightarrow$ & \multicolumn{1}{c|}{$\begin{array}{c}\text { Strongly } \\
\text { Agree }\end{array}$} \\
\hline $\begin{array}{l}\text { This intervention has a high degree of } \\
\text { scientific merit }\end{array}$ & 1 & 2 & 3 & 4 & 5 & 6 & 7 & 8 & 9 \\
\hline $\begin{array}{l}\text { Such an intervention has the potential for } \\
\text { significant impact in nursing homes }\end{array}$ & 1 & 2 & 3 & 4 & 5 & 6 & 7 & 8 & 9 \\
\hline \multicolumn{7}{|l|}{} \\
\hline $\begin{array}{l}\text { This intervention is feasible for nursing } \\
\text { homes }\end{array}$ & 1 & 2 & 3 & 4 & 5 & 6 & 7 & 8 & 9 \\
\hline $\begin{array}{l}\text { Such an intervention is useful } \\
\text { for accountability purposes }\end{array}$ & 1 & 2 & 3 & 4 & 5 & 6 & 7 & 8 & 9 \\
\hline $\begin{array}{l}\text { Implementation of this intervention in } \\
\text { nursing homes is important }\end{array}$ & 1 & 2 & 3 & 4 & 5 & 6 & 7 & 8 & 9 \\
\hline
\end{tabular}

Additional Comments: 


\section{Intravenous (IV) to Oral Transition}

Description: Guideline implementation to transition antibiotics from IV to oral when clinically indicated.

\section{IV to Oral Transition}

Instructions: Please rate your agreement in applying each of the following criteria on a 9-point scale (1 - Strongly Disagree, 9 - Strongly Agree)

\begin{tabular}{|c|c|c|c|c|c|c|c|c|c|}
\hline & $\begin{array}{l}\text { Strongly } \\
\text { Disagree }\end{array}$ & $\rightarrow$ & $\rightarrow$ & $\rightarrow$ & $\Rightarrow$ & $\rightarrow$ & $\rightarrow$ & $\rightarrow$ & $\begin{array}{l}\text { Strongly } \\
\text { Agree }\end{array}$ \\
\hline $\begin{array}{l}\text { This intervention has a high degree of } \\
\text { scientific merit }\end{array}$ & 1 & 2 & 3 & 4 & 5 & 6 & 7 & 8 & 9 \\
\hline $\begin{array}{l}\text { Such an intervention has the potential for } \\
\text { significant impact in nursing homes }\end{array}$ & 1 & 2 & 3 & 4 & 5 & 6 & 7 & 8 & 9 \\
\hline $\begin{array}{l}\text { This intervention is feasible for nursing } \\
\text { homes }\end{array}$ & 1 & 2 & 3 & 4 & 5 & 6 & 7 & 8 & 9 \\
\hline $\begin{array}{l}\text { Such an intervention is useful } \\
\text { for accountability purposes }\end{array}$ & 1 & 2 & 3 & 4 & 5 & 6 & 7 & 8 & 9 \\
\hline $\begin{array}{l}\text { Implementation of this intervention in } \\
\text { nursing homes is important }\end{array}$ & 1 & 2 & 3 & 4 & 5 & 6 & 7 & 8 & 9 \\
\hline
\end{tabular}

Additional Comments: 


\section{Multifaceted Interventions}

Description: Bundling of two or more ASP intervention strategies to form a complex intervention. Generally educational interventions combined with audit and feedback and/or guidelines. Can include physician e-learning modules, decision support, and engagement of patients.

\section{Multifaceted Interventions}

Instructions: Please rate your agreement in applying each of the following criteria on a 9-point scale (1 - Strongly Disagree, 9 - Strongly Agree)

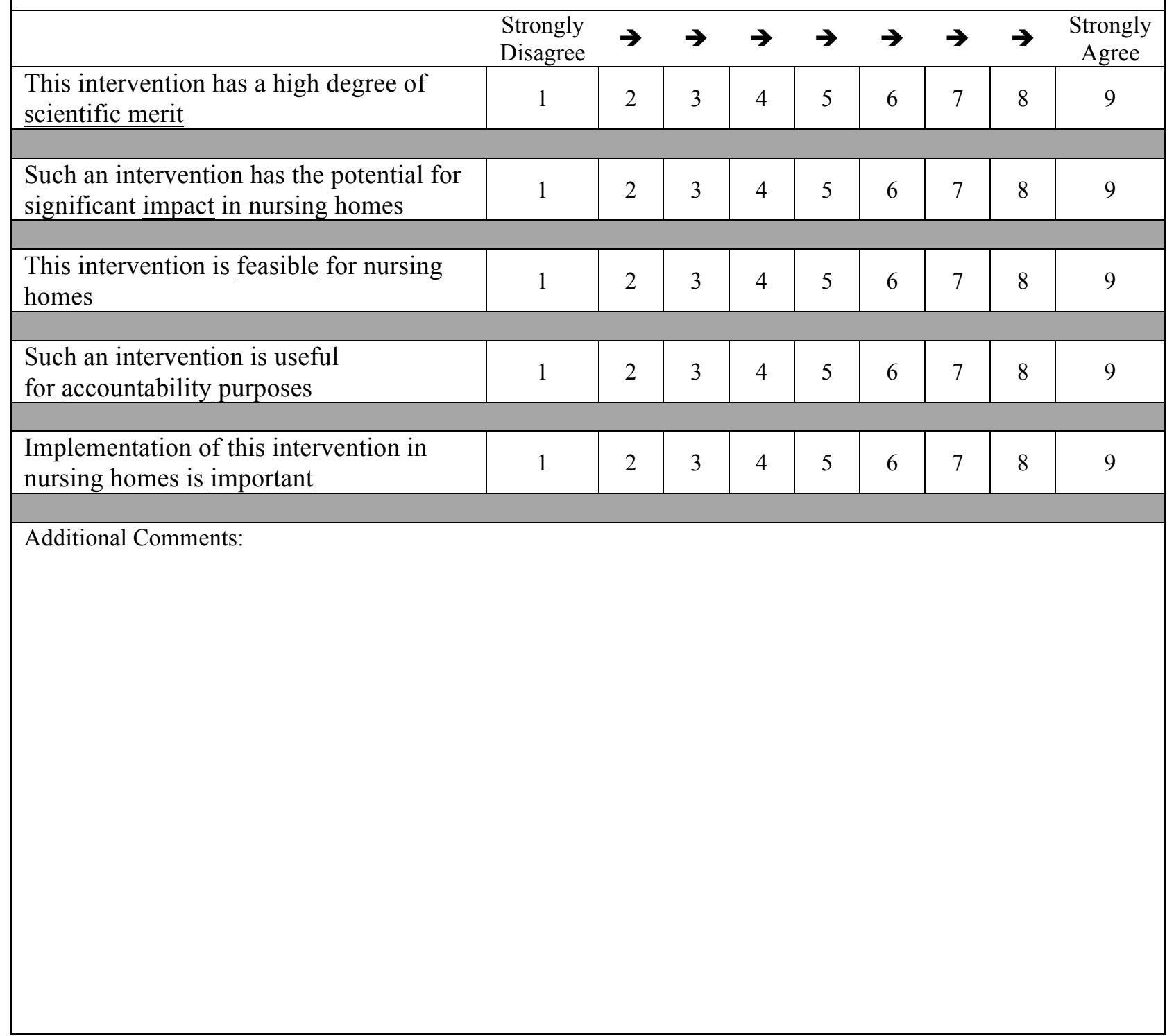


Short Course Antibiotic Therapy

Description: Implementation of guidelines encouraging short durations of antibiotic therapy for specific uncomplicated infections.

\section{Short Course Antibiotic Therapy}

Instructions: Please rate your agreement in applying each of the following criteria on a 9-point scale (1 - Strongly Disagree, 9 - Strongly Agree)

\begin{tabular}{|c|c|c|c|c|c|c|c|c|c|}
\hline & $\begin{array}{l}\text { Strongly } \\
\text { Disagree }\end{array}$ & $\rightarrow$ & $\rightarrow$ & $\Rightarrow$ & $\rightarrow$ & $\rightarrow$ & $\rightarrow$ & $\rightarrow$ & $\begin{array}{c}\text { Strongly } \\
\text { Agree }\end{array}$ \\
\hline $\begin{array}{l}\text { This intervention has a high degree of } \\
\text { scientific merit }\end{array}$ & 1 & 2 & 3 & 4 & 5 & 6 & 7 & 8 & 9 \\
\hline $\begin{array}{l}\text { Such an intervention has the potential for } \\
\text { significant impact in nursing homes }\end{array}$ & 1 & 2 & 3 & 4 & 5 & 6 & 7 & 8 & 9 \\
\hline $\begin{array}{l}\text { This intervention is feasible for nursing } \\
\text { homes }\end{array}$ & 1 & 2 & 3 & 4 & 5 & 6 & 7 & 8 & 9 \\
\hline $\begin{array}{l}\text { Such an intervention is useful } \\
\text { for accountability purposes }\end{array}$ & 1 & 2 & 3 & 4 & 5 & 6 & 7 & 8 & 9 \\
\hline $\begin{array}{l}\text { Implementation of this intervention in } \\
\text { nursing homes is important }\end{array}$ & 1 & 2 & 3 & 4 & 5 & 6 & 7 & 8 & 9 \\
\hline
\end{tabular}

Additional Comments: 


\section{Targeting Clostridium Difficile Infection (CDI)}

Description: Increasing appropriate therapy and adherence to guidelines for treating CDI. Includes education, guidelines, and restriction components.

\section{Targeting Clostridium Difficile Infection (CDI)}

Instructions: Please rate your agreement in applying each of the following criteria on a 9-point scale (1 - Strongly

Disagree, 9 - Strongly Agree)

\begin{tabular}{|c|c|c|c|c|c|c|c|c|c|}
\hline & $\begin{array}{l}\text { Strongly } \\
\text { Disagree }\end{array}$ & $\rightarrow$ & $\rightarrow$ & $\rightarrow$ & $\rightarrow$ & $\rightarrow$ & $\rightarrow$ & $\rightarrow$ & $\begin{array}{l}\text { Strongly } \\
\text { Agree }\end{array}$ \\
\hline $\begin{array}{l}\text { This intervention has a high degree of } \\
\text { scientific merit }\end{array}$ & 1 & 2 & 3 & 4 & 5 & 6 & 7 & 8 & 9 \\
\hline $\begin{array}{l}\text { Such an intervention has the potential for } \\
\text { significant impact in nursing homes }\end{array}$ & 1 & 2 & 3 & 4 & 5 & 6 & 7 & 8 & 9 \\
\hline $\begin{array}{l}\text { This intervention is feasible for nursing } \\
\text { homes }\end{array}$ & 1 & 2 & 3 & 4 & 5 & 6 & 7 & 8 & 9 \\
\hline $\begin{array}{l}\text { Such an intervention is useful } \\
\text { for accountability purposes }\end{array}$ & 1 & 2 & 3 & 4 & 5 & 6 & 7 & 8 & 9 \\
\hline $\begin{array}{l}\text { Implementation of this intervention in } \\
\text { nursing homes is important }\end{array}$ & 1 & 2 & 3 & 4 & 5 & 6 & 7 & 8 & 9 \\
\hline
\end{tabular}

Additional Comments: 


\section{Other Comments:}

Additional comments - including ASP interventions that have been left out of this package entirely and you would like to see included or discussed on the day of the panel 


\section{Appendix C. Conflict of Interest Form}

\section{Potential Conflicts of Interest}

Modified Delphi panel to evaluate

Antimicrobial Stewardship interventions in nursing homes

March 20, 2018

Full Name:

Please comment on your financial or other relationships with entities that could be perceived to influence, or that give the appearance of potentially influencing your contributions with the Modified Delphi Panel to evaluate Antimicrobial Stewardship interventions in nursing homes.

We have received funding for this project from the Canadian Frailty Network. 


\section{Appendix D. Delphi Meeting Agenda}

\section{Agenda}

ASP Delphi Meeting - Evaluating ASPs for Nursing Homes

Location: 60 Murray Street Toronto, Ontario

Date: March 20, 2018

Time Item

9:00am: Welcome

Introductions

Discuss Consensus Goals

9:30am: Review results from survey

Begin discussion of interventions for ASPs

Second round of survey

12:45pm: Lunch

1:15pm: Continue discussion

Discuss resourcing for interventions

3:45pm: Prioritize interventions

4:10pm: $\quad$ End of Day Survey

4:20pm: Closing comments

4:30pm: Panel completed

Time: 9:00am -

4:30pm 


\title{
Appendix E. End of Day Survey
}

\author{
End of Day Survey \\ ASP Delphi Meeting \\ Evaluating ASPs for Nursing Homes \\ March 20, 2018
}
Pre-meeting
1) Background information provided offered enough support
2) Instructions were clear and easy to follow
3) Expectations were well laid out
4) In your opinion, the questionnaire took an appropriate amount of time to complete
5) You were able to understand and complete all questions
6) The format in which the package were received was easy and unobtrusive

Strongly

Disagree

Disagree

Somewh

Agree

Strongly at Agree Agree

\section{In-person meeting}

Throughout the discussions did you feel:

7) Your opinion mattered

8) You were ever disrespected

9) Your voice was heard and you had a chance to share your thoughts

10) Too much time was given to any single other panel member

11) You were able to follow and understand the discussion at all times

When completing the second sets of questions, did you:

12) Change any of your previous answers because you felt intimidated to do so

13) Keep any of your previous answers because you did not understand the discussion

14) Feel as though your safety or reputation were at stake 
Additional Comments:

Please share your opinions and additional comments below:

Was there anything that was not addressed today you wish had been?

Where do you see the research related to this area of topic heading to?

What, if anything, would you change from the way this panel has been organized and conducted?

Additional comments: 


\section{Appendix F. Literature Search Strategy}

Database: All Ovid MEDLINE(R) <1946 to Present> Search Strategy:

1 Antimicrobial Stewardship/ (57)

2 (stewardship adj2 (antimicrobial or anti-microbial or antibiotic or anti-biotic)).kf,tw. (2733)

31 or $2(2749)$

4 Long-Term Care/ (24061)

5 (nursing home* or long-term care or residential care or care facilit*).kf,tw. (55541)

64 or $5(70579)$

73 and $6(158)$

8 intervention*.mp. (803381)

97 and $8(45)$

10 limit 9 to (guideline or meta analysis or practice guideline or "review" or "scientific integrity review" or systematic reviews) (14)

11 Homes for the Aged/ (12663)

124 or 5 or $11(76478)$

133 and 8 and $12(46)$

14 limit 13 to (guideline or meta analysis or practice guideline or "review" or "scientific integrity review" or systematic reviews) (15)

15 from 14 keep $1-15$ (15) 


\section{Appendix G. Delphi Meeting - Survey 1 Results}

Table 1. ASPs - Survey results describing scores for interventions across five evaluative criteria

\begin{tabular}{|c|c|c|c|c|c|}
\hline Interventions & $\begin{array}{c}\text { Scientific } \\
\text { Merit }\end{array}$ & Impact & Feasibility & Accountability & Importance \\
\hline Antibiograms & $53 \%$ & $60 \%$ & $53 \%$ & $47 \%$ & $60 \%$ \\
\hline Antibiotic Cycling & $20 \%$ & $13 \%$ & $20 \%$ & $13 \%$ & $13 \%$ \\
\hline $\begin{array}{c}\text { Audit and } \\
\text { feedback }\end{array}$ & $60 \%$ & $87 \%$ & $53 \%$ & $60 \%$ & $67 \%$ \\
\hline $\begin{array}{l}\text { Clinical decision } \\
\text { support system }\end{array}$ & $60 \%$ & $87 \%$ & $47 \%$ & $60 \%$ & $67 \%$ \\
\hline De-escalation & $67 \%$ & $67 \%$ & $53 \%$ & $53 \%$ & $67 \%$ \\
\hline Education & $60 \%$ & $67 \%$ & $67 \%$ & $47 \%$ & $73 \%$ \\
\hline $\begin{array}{c}\text { Formulary } \\
\text { automatic } \\
\text { substitution }\end{array}$ & $22 \%$ & $33 \%$ & $33 \%$ & $20 \%$ & $20 \%$ \\
\hline $\begin{array}{l}\text { Formulary } \\
\text { restriction }\end{array}$ & $47 \%$ & $67 \%$ & $40 \%$ & $47 \%$ & $47 \%$ \\
\hline Formulary review & $33 \%$ & $40 \%$ & $47 \%$ & $33 \%$ & $47 \%$ \\
\hline $\begin{array}{c}\text { Guidelines for } \\
\text { empiric } \\
\text { prescribing }\end{array}$ & $80 \%$ & $87 \%$ & $80 \%$ & $73 \%$ & $87 \%$ \\
\hline $\begin{array}{l}\text { IV to oral } \\
\text { transition }\end{array}$ & $33 \%$ & $40 \%$ & $40 \%$ & $40 \%$ & $27 \%$ \\
\hline Multifaceted & $87 \%$ & $93 \%$ & $33 \%$ & $40 \%$ & $73 \%$ \\
\hline $\begin{array}{c}\text { Short course } \\
\text { antibiotic therapy }\end{array}$ & $67 \%$ & $80 \%$ & $87 \%$ & $73 \%$ & $87 \%$ \\
\hline Targeting CDI & $60 \%$ & $60 \%$ & $60 \%$ & $53 \%$ & $60 \%$ \\
\hline
\end{tabular}

Legend: Colour Coding Cut-Offs

Percentages represent scores ranging between 7-9 in survey for each criterion

Percentages $\geq 75 \%$ - $\square$ (Agreement)

Percentages $50-74 \%$ - $\square$ (Uncertainty)

Percentages $<50 \%$ - $\square$ (Disagreement) 


\section{Appendix H. Contributions}

The completion of this thesis is due to the contributions of many. Dr. Chaim Bell provided extensive time and effort to this thesis in its every stage. Dr. Andrew Morris helped organize and was the facilitator for our Modified Delphi panel. My program advisory committee, Dr. Bronskill and Dr. Jeffs, deserve much recognition for brainstorming ideas for this thesis and ultimately assisting us in the decision to conduct a Modified Delphi panel.

The Modified Delphi Method is nothing without the panelists: Dr. Carla Beaton, Ms. Anne Bialachowski, Ms. Sharron Cooke, Dr. Nick Daneman, Dr. Kamyab Ghatan, Ms. Devora Greenspon, Dr. Lynn Johnston, Dr. Louis Kennedy, Ms. Maloree Kubica, Mr. Jonathan Lam, Dr. Bradley Langford, Ms. Dee Lender, Dr. Justin Lin, Dr. Mark Loeb, Dr. Lindsay Nicolle, and Dr. Lisa Sever.

This project was funded by the Canadian Frailty Network (formerly Technology Evaluation in the Elderly Network (TVN)), as well as a 2017-2018 Ontario Student Opportunity Trust Fund Award provided by the Lunenfeld Tananbaum Research Institute Scholarships at the Sinai Health System. 


\section{Copyright Acknowledgements}

Figure 2 was reproduced with permission from the U.S. GRADE Network. A copy of the email correspondence can be seen below.

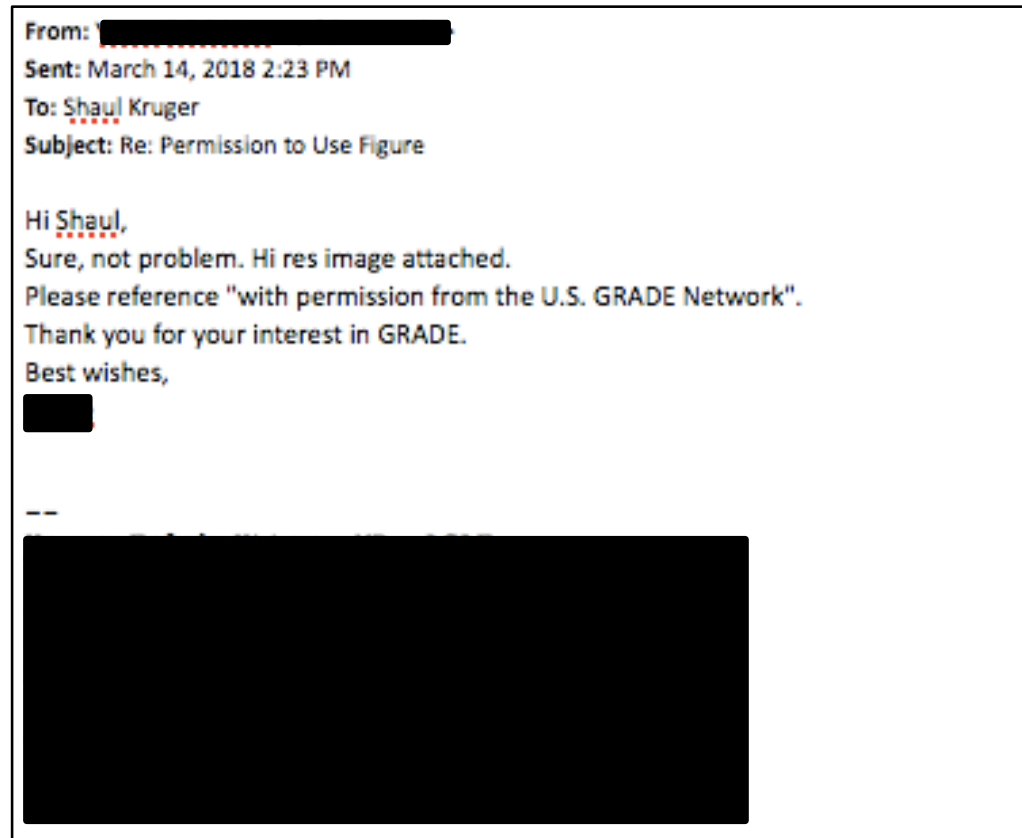

Figure 3 was reproduced with permission from the RAND Corporation. A copy of the email correspondence can be seen below.

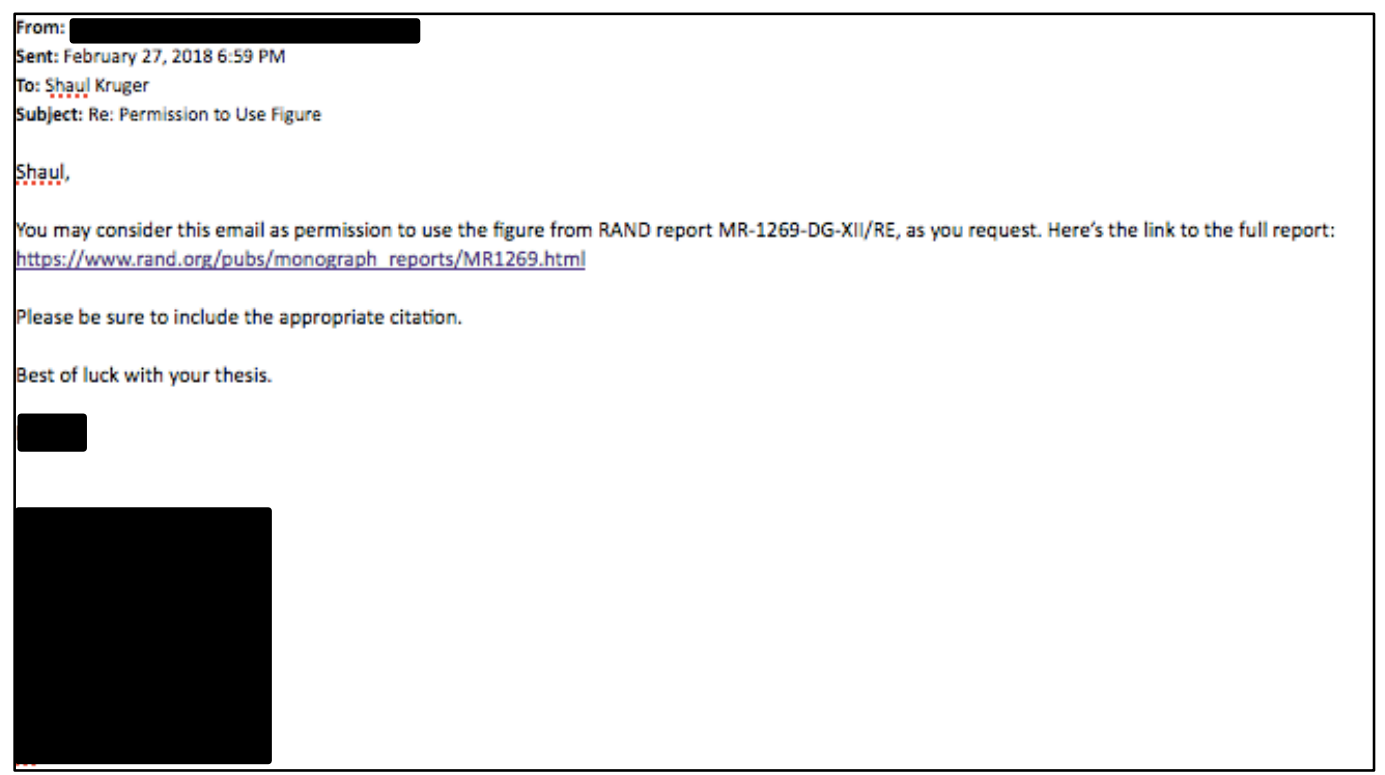

